\title{
Public Health Genomics : translation of genomic research on smoking behaviour into evidence synthesis and guidelines for public health
}

Citation for published version (APA):

de Viron, S. (2013). Public Health Genomics : translation of genomic research on smoking behaviour into evidence synthesis and guidelines for public health. [Doctoral Thesis, Maastricht University]. Maastricht University. https://doi.org/10.26481/dis.20131206sv

Document status and date:

Published: 01/01/2013

DOI:

10.26481/dis.20131206sv

Document Version:

Publisher's PDF, also known as Version of record

Please check the document version of this publication:

- A submitted manuscript is the version of the article upon submission and before peer-review. There can be important differences between the submitted version and the official published version of record.

People interested in the research are advised to contact the author for the final version of the publication, or visit the DOI to the publisher's website.

- The final author version and the galley proof are versions of the publication after peer review.

- The final published version features the final layout of the paper including the volume, issue and page numbers.

Link to publication

\footnotetext{
General rights rights.

- You may freely distribute the URL identifying the publication in the public portal. please follow below link for the End User Agreement:

www.umlib.nl/taverne-license

Take down policy

If you believe that this document breaches copyright please contact us at:

repository@maastrichtuniversity.nl

providing details and we will investigate your claim.
}

Copyright and moral rights for the publications made accessible in the public portal are retained by the authors and/or other copyright owners and it is a condition of accessing publications that users recognise and abide by the legal requirements associated with these

- Users may download and print one copy of any publication from the public portal for the purpose of private study or research.

- You may not further distribute the material or use it for any profit-making activity or commercial gain

If the publication is distributed under the terms of Article 25fa of the Dutch Copyright Act, indicated by the "Taverne" license above, 
PUBLIC HEALTH GENOMICS:

TRANSLATION OF GENOMIC RESEARCH ON SMOKING BEHAVIOUR INTO EVIDENCE SYNTHESIS AND GUIDELINES FOR PUBLIC HEALTH

SYLVIANE DE VIRON 
(C) Sylviane de Viron, Maastricht 2013

Layout: Sylviane de Viron

Cover: Sylviane de Viron

Printing: Smart Printing Solutions

ISBN: 9789090278995

Legal deposit: D/2013/2505/35

The research presented in this thesis was conducted at the School for Oncology and developmental biology: GROW, Department of Genetics \& cell biology, of Maastricht University. The Belgian Federal Science Policy Office funded this thesis. 
PUBLIC HEALTH GENOMICS:

TRANSLATION OF GENOMIC RESEARCH ON

SMOKING BEHAVIOUR INTO EVIDENCE

SYNTHESIS AND GUIDELINES FOR PUBLIC HEA LTH

\section{DisSERTATION}

to obtain the degree of Doctor at Maastricht University, on the authority of the Rector Magnificus, Prof. Dr. L.L.G. Soete, in accordance with the decision of the Board of Deans, to be defended in public on Friday 6 December 2013 at 12.00 hours by 
SUPERVISORS:

Prof. Dr. A. Brand MPH, Professor of Social Medicine \& Public Health Genomics, Founding Director of the Institute for Public Health Genomics, Department of Genetics and Cell Biology, Faculty of Health Medicine and Life Sciences, Maastricht University

Dr. TMA Pai Endowed Chair in Public Heath Genomics and Adjunct Professor, Manipal Life Sciences Center, Manipal University, India

Prof. Dr. H. Van Oyen MPH, Professor of Epidemiology, OD Public Health and Surveillance, Scientific Institute of Public Health, Belgium

Department of Public health, University of Ghent, Belgium

Prof. Dr. S.A. Morré, Professor of Host-Pathogen Genomics and Public Health, Director of the Institute for Public Health Genomics, Department of Genetics and Cell Biology, Faculty of Health Medicine and Life Sciences, Maastricht University Head of the Laboratory of Immunogenetics, Department of Medical Microbiology and Infection Control, VU University Medical Center, Amsterdam

ASSESSMENT COMMITTEE:

Prof. Dr. J. Glatz (UM) (Chair)

Prof. Dr. B. Smeets (UM)

Em. Prof. Dr. J.J. Cassiman (Catholic University of Leuven, Belgium)

Prof. Dr. S. Van den Broucke (Catholic University of Louvain, Belgium) 
If you understand what you are doing, you are not learning anything

- Unknown 



\section{CONTENTS}

Chapter $1 \quad$ INTRODUCTION 3

Part i IMPROVING HeALTHCARE FOR SMOKERS 19

Chapter 2 GENETIC PREDICTORS FOR SMOKING INITIATION AND CESSATION 21

Chapter 3 ENVIRONMENTAL AND GENOMIC FACTORS AS WELL AS INTERVENTIONS INFLUENCING SMOKING CESSATION: A SYSTEMATIC REVIEW OF REVIEWS AND A PROPOSED WORKING MODEL 35

Chapter 4 GENETIC SIMILARITIES BETWEEN COMORBIDITIES OF TOBACCO USE DISORDERS: AN EXPLORATORY STUDY

Part ii ENHANCING THE COMMUNiCATION OF GENOMiC Risks About SMOKING IN THE GENERAL POPULATION 73

Chapter 5 IMPACT OF GENETIC NOTIFICATION ON SMOKING CESSATION 75

Chapter 6 COMMUNICATING GENETICS AND SMOKING THROUGH SOCIAL MEDIA: ARE WE THERE YET? 93

Chapter 7 WHICH INTERNET TOOLS ARE SEARCHED BY STUDENTS AT UNIVERSITY TO OBTAIN INFORMATION ABOUT HEALTH, SMOKING AND GENETICS? A PILOT STUDY 109

Chapter $8 \quad$ GENERAL DISCUSSION 121

\begin{tabular}{|c|c|c|}
\hline \multicolumn{3}{|c|}{ SUMMARY 131} \\
\hline \multicolumn{3}{|c|}{ SAMENVATTING } \\
\hline RÉSUMÉ & & \\
\hline \multicolumn{2}{|c|}{ ACKNOWLEDGEMENTS } & 141 \\
\hline \multicolumn{2}{|c|}{ CURRICULUM VITAE } & 142 \\
\hline PUBLICATIONS & 143 & \\
\hline REFERENCES & & \\
\hline
\end{tabular}




\section{LIST OF FIGURES}

Figure 1.1

Figure 1.2

Figure 1.3

Figure 2.1

Figure 2.2

Figure 2.3

Figure 3.1

Figure 3.2

Figure 4.1

Figure 4.2

Figure $4 \cdot 3$

Figure 5.1

Figure 5.2

Figure $5 \cdot 3$

Figure 5.4

Figure 6.1

Figure 6.2

Figure 6.3

Figure 7.1
The Public Health Genomics enterprise 9

The integration of genomics into public health 10

Phenotype choice points along the smoking trajectory

Schematic diagram of the multifactorial factors influencing smoking initiation and cessation 23

Genetic pathways influencing smoking initiation and cessation 25

Schematic diagram of the hierarchy in the '-omics' 32

Flow chart of the study selection process 40

Working model of Smoking Cessation 49

Flow of the analyses 61

Genetic network analyses using Ingenuity Pathway Analysis and STRING 64

Dendrogram of disorders associated with the same genes as TUD resulting from centroid hierarchical cluster analysis, using the Jaccard distance obtained from 32 genes 66

Flow chart of the study selection process $\quad 79$

Comparison of the distributions (High, low genetic risk and/or control) between studies or genes $\quad 85$

Pooled-analysis of smoking cessation associated with genetic notification in randomised trials for the last follow-up $(2,6$ or 12 month) 86

Pooled-analysis of smoking cessation associated with genetic notification in randomised trials for follow-up $\leqslant 6$ month $(2$ or 6 month) 87

Screenshots of YouTube and Twitter results $\quad 97$

Flow Chart of the post selection 99

Word cloud presenting the most used words on YouTube, Twitter, and Google 103

Flow diagram of data of the study exploring the Internet tools used by students at the Catholic University of Louvain to search for information about health, smoking and genetics 114 
Table 1.1

Table 2.1

Table 2.2

Table 3.1

Table 3.2

Table $3 \cdot 3$

Table 4.1

Table 4.2

Table $4 \cdot 3$

Table 5.1

Table 5.2

Table $5 \cdot 3$

Table 6.1

Table 6.2

Table 7.1

Table 7.2

Table $7 \cdot 3$
Essential services for PHG 11

Allele frequency of $D R D 2$ C957T 24

Candidate genes with associations reported at least once in the literature 29

Overall results of the selected meta-analyses $\quad 41$

Genes influencing SC and reported interactions with interventions 45

Description of the included studies for the genomic review

Genes associated with tobacco use disorder selected from Gene2Mesh and Ingenuity 62

Disorders associated with genes influencing tobacco use disorder selected from methods 1 and $2 \quad 65$

Jaccard distance between pairs of disorders associated with genes influencing tobacco use disorder $\quad 67$

Genetic notification and smoking cessation: Overview of included studies $\quad 82$

Risk ratios and 95\% confidence intervals associated with smoking cessation following intervention (genetic notification versus control) 85

Quality assessment of studies included in the systematic review of genetic notification 91

Characteristics of included posts on YouTube and Twitter 100 Comparison of the content of the posts between Time 1 and 2102

General characteristics of the student's population by searching or not for information about at least health, smoking or genetics in Internet tools 115

Types of internet-based tools used to obtain information about health, smoking and genetics 116

Multivariate logistic regressions about the use of Internet tool to search for information about health, smoking and genetics 116 
MAJOR ABBREVIATIONS

\begin{tabular}{ll} 
ADHD & Attention Deficit Disorder with Hyperactivity \\
COPD & Chronic Obstructive Pulmonary Disease \\
DNA & Deoxyribonucleic Acid \\
GABA & Gamma Aminobutyric Acid \\
GWAS & Genome Wide Association study \\
HWE & Hardy Weinberg Equilibrium \\
mRNA & Messenger Ribonucleic Acid \\
nAChRs & Nicotinic Acetylcholine Receptors \\
NAcc & Nucleus Accumbens \\
NRT & Nicotine Replacement Therapy \\
PHG & Public Health Genomics \\
PHGEN & Public Health Genomics European Network \\
RDS & Reward Deficiency Syndrome \\
SES & Socioeconomic Status \\
SNP & Single Nucleotide Polymorphism \\
TUD & Tobacco Use Disorder \\
VTA & Ventral Tegmental Area \\
\hline
\end{tabular}




INTRODUCTION 
Public Health Genomics

Public Health Genomics mission

Causation of complex disorders

The necessity of translation

The scope of Public Health Genomics

Two working concepts for Public Health Genomics

The Public Health Genomics enterprise

The wheel of the ten essential Public Health Genomics activities Application of Public Health Genomics on smoking

Introduction

Smoking and Public Health Genomics

Translation of genomic research on smoking

Communication of genome-based information about smoking

Aims and outline of the thesis 
Genomic research and knowledge rapidly grew since the completion of the $\mathrm{Hu}-$ man Genome Project in 2003. Different areas of genomic research have been developed from bench to bedside and beyond from bench to healthcare including, for example, fundamental research in systems biology, the Personal Genome Project, molecular epidemiology and new challenges for Public Health Genomics (PHG), personalised, and individualised healthcare.

In 1997 the institutionalisation of PHG started with the establishment of the Office of Public Health Genomics within the Centre for Disease Control and Prevention (CDC) in the USA and with the foundation of the Unit of Public Health Genomics within Cambridge University in the UK. At the same time, PHG was established as a new field in Germany as well. However, it was not institutionalised. In 2003, the first international cooperation group followed by the first international meeting on PHG took place in Bielefeld, Germany and was coordinated by Prof. Dr. Angela Brand.

PHG, as defined during the Bellagio workshop in 2005, in which all of the above mentioned groups participated, is 'the responsible and effective translation of genomebased knowledge and technologies into public policy and health services for the benefit of population health' $[1,2]$. According to that definition, the major aim of PHG is to translate research from basic sciences into health policies and healthcare to improve population health. To date, the most important problem is the lack of translation from basic research to the healthcare system, as only around $2 \%$ of research is effectively translated based on the amount of funding and activities [3]. In Europe, the Public Health Genomics European Network (PHGEN), funded by the General Directorate for Health and Consumer Protection (DG SANCO), from the very beginning specifically worked on the translating knowledge 'from cell to society'. The second phase of the PHGEN project ended with the first edition of 'European Best Practice Guidelines for Quality Assurance, Provision and Use of Genome-based Information and Technologies' [4]. These guidelines have been endorsed in 2012 by key European institutions and organisations like the European Medicines Agency (EMA) and the European Science Foundation (ESF) as well as by the European Member States. They will guide the Member States within the next few years in the field of PHG and personalised healthcare.

This thesis uses smoking as a case study to apply PHG. Smoking is still a major public health problem worldwide despite the widespread knowledge of the risks of morbidity and mortality. Both, preventing smoking initiation and enhancing smoking cessation are the best way to reduce risks of smoking related disorders. In a public health point of view, different approaches are used to prevent smoking initiation and enhance smoking cessation such as taxation, bans and media campaigns [5]. However, these interventions have shown some limitations in reducing smoking prevalence. Therefore, genomics may be an innovative and useful way to promote public health. Although no applications are currently ready for practice, potential applications of genomics are investigated in the prevention, the control and the cessation of smoking. It is why including genomic knowledge is a more holistic and 
useful approach leading to personalised interventions taking the advantage of the supportive environment [5]. Interventions may include (1) genomic risk notification of smoking initiation, nicotine addiction, smoking cessation or smoking related disorders or (2) genomically adapted treatments in drugs, dose and duration, which may enhance both personalised and individualised treatments [6].

The aim of the present thesis is to apply PHG on smoking. To achieve this objective, two main goals were developed: firstly, to improve healthcare for smokers, and secondly to propose new approaches to enhance the communication of genomic risks about smoking in the general population.

The general introduction of this thesis starts with theories on PHG. After that, the application of PHG concepts on smoking is developed.

\section{PUBLIC HEALTH GENOMICS}

\section{Public Health Genomics mission}

The initial idea of PHG is to devise effective preventive interventions targeted at individuals with specific genotypes [7]. Its main attempt would, therefore, not primarily concern the new technological advances like gene therapy [8] but subcategorise populations compatible to the effectiveness of preventive environmental interventions stratified according to their genetic risk. The motivation behind this strategy is that the molecular and cellular processes, which are encoded in the gene sequence, are susceptible to influence from the external environment.

\section{Causation of complex diseases - Role of epidemiology}

At this moment, however, clear applications of PHG in the prevention of disorders remain distant. We still do not understand the complexity of diseases in the causation of a certain common disorder. Very few common genetic variants are known to increase the risk of complex disorders substantially [9]. Even when a genetic risk is substantial, knowledge of the risk contributes to improved health outcomes only if effective measures are available for preventive or early treatment [2]. Similarly, genetic testing is of uncertain value when the available interventions are not genotype-specific. Furthermore, for a genetic test to be useful in the management of a common disorder, it must have predictive powers over and above accepted risk factors, which can easily be measured [9]. Genetic testing will have its greatest public health value when it identifies individuals who would benefit from specific interventions based on their risk [2, 7]. However, the major limitation is that genetic tests mostly do not take the environment into account.

To better understand the cause of complex disorders by taking notice of the genetic contribution is one of the main thrusts of genetic epidemiology [10]. To date, most proposed advances resulted from genetic epidemiological research using methods like the investigation of familial clustering, twin, adoption, and migration studies. The specific genetic determinants have been identified through linkage 
analysis and, more recently, through pathway analyses and genome-wide association studies (GWAS). A GWAS is defined as a study of common genetic variation across the entire human genome designed to identify genetic associations with observable traits [11]. These studies use high-throughput genotyping technologies to assay hundreds of thousands of single nucleotide polymorphisms (SNPs) and relate these genetic variants to disorders or health-related traits [11]. However, a GWAS is prone to false-positive and false-negative associations, which demands for the replication of the study results in an independent population sample [11].

Although the GWAS often offer robust associations between certain SNPs and complex disorders, the population implications of those findings are mostly unclear because the basic population-based genetic prevalence data are mostly unevaluated [12]. Population-based research is essential to quantify the population prevalence of genetic variants, the magnitude of disorder risk associated with these variants (in relative and absolute terms), the contribution of these variants to the occurrence of disorder in different populations (attributable risk), the existence of geneenvironment and gene-gene interactions and the validity of genetic tests based on such variants in predicting disorder risks [13, 14]. The Human Genome Epidemiology Network (HuGENet) is a global collaboration of individuals and organisations to assess population health impact of human genomic variation and how it can be used to improve health and prevent disorders in populations. Their main aim is to conduct rigorous systematic, peer-reviewed reviews and meta-analyses of genetic associations and of the epidemiological aspects of variation in particular genetic variants, the HuGE reviews [15].

The necessity of translation

Obviously, the relation between genes and a phenotype is far from simple. Thus, there is a need to translate the information about genes into information about genomic susceptibility for disorders, the interaction between these susceptibilities and the impact of this knowledge on population health. Khoury et al. presented a framework for the continuum of multidisciplinary translation research in genomic medicine [3]. The purpose is to move promising genomic applications to clinical and public health practice for population health benefit. The framework consists of four phases from $\mathrm{T}_{1}$ to $\mathrm{T}_{4}$. $\mathrm{T}_{1}$ : gene discovery to candidate health application; T2: health application to evidence-based practice guidelines; $\mathrm{T}_{3}$ : practice guidelines to health practice and $\mathrm{T}_{4}$ : practice to population health impact [3]. It is based on genomic epidemiology findings.

Lal et al. also proposed a new model (Learning Adapting Leveling, LAL model) aiming to enhance the translation and mostly focusing on the translation of genomebased technologies. This model includes both the technology transfer activity and public health assessment technologies in relationship to public-private partnerships to promote relevant technologies. Currently, the LAL model is the most holistic approach, since it covers the whole pipeline 'from cell to society', i.e. from basic sciences into implementation in healthcare systems [16]. 
Although moving scientific discoveries into practice and the delivery of population-level health benefit has always been slow and difficult at best, many believe that this is a doable project when all relevant stakeholders on all levels are involved [9].

The broader scope of Public Health Genomics

PHG is a task and has a broader scope than only biomedical sciences like the molecular research, pharmacogenomics or genomic epidemiology. It aims to achieve the understanding of systems medicine, which incorporates both environmental and genomic factors, to promote truly personalised healthcare based on the individual rather than populations of patients. This also implies future modifications in disease classifications based on diseasomes [17]. One of the challenges in the evaluation of genomic applications to healthcare is the integration of studies of the ethical, legal, and social implications (ELSI). A 2003 report by the Institute of Medicine in the US identified genomics as a priority for the training of all public health professionals in the $21^{\text {st }}$ century [18]. Therefore, the public health community has a major role to play in raising the level of general genomic literacy, developing targeted messages about the uses of genome-based information in disorder prevention and coordinating communication strategies with stakeholder groups [10].

\section{Two working concepts for Public Health Genomics}

All these concepts are grouped together in two concepts where the PHG research can be fit in: the PHG enterprise and the wheel of the ten essential PHG functions.

The Public Health Genomics enterprise

This enterprise was developed on consensus by the international key experts of the Bellagio meeting to demonstrate how to translate genome-based science and technology, together with the human sciences, into improvements in population health $[1,2]$. Figure 1.1 shows the PHG enterprise. It represents a way of working or approaching problems, rather than a discrete subject that includes certain topics and excluded others. This means that any new development in modern genomics or in molecular and cell biology fits within the scope of the enterprise. Figure 1.1 shows the fundamental role of genome-based science and technology and the achievement of improvement in population health as an ultimate goal [1, 2]. Besides the genomebased science and technology, the enterprise acknowledges the need to incorporate research and knowledge from the population sciences and from the humanities and social sciences as important inputs. Knowledge integration plays a central role, not only within but also across disciplines and is therefore the driving force of the PHG enterprise. It is defined as the process of selecting, storing, collecting, analysing, integrating, and disseminating information both within and across disciplines for the 
benefit of population health. It further includes the methodological developments too, and is the means by which information is transformed into useful knowledge.

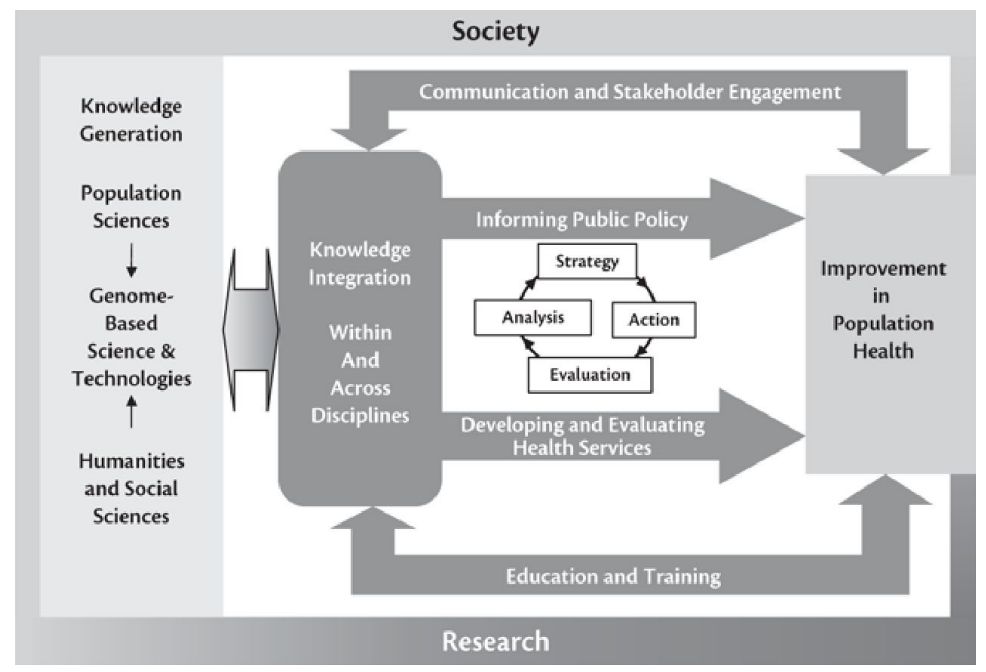

Figure 1.1: The Public Health Genomics enterprise (Adapted from the report of an expert workshop held at the Rockefeller Foundation Study and Conference Centre Bellagio, Italy, $2005[1])$

The integrated multidisciplinary knowledge generated from an effective PHG enterprise supports four core activities. First, informing public policy includes the whole range of legal, philosophical, and social analyses, development of regulatory frameworks, engagement in the policy-making process, promoting relevant research, seeking international comparisons, and working with governments and relevant public institutions. Second, developing and evaluating preventive and clinical health services includes development of policies, programmes and services in the health sector, strategic planning, service organisation, manpower planning and capacity building, service review and evaluation and guideline development. The third core activity of the enterprise is communication and stakeholder engagement. Relevant activities include promoting general genomic literacy in society, public dialogue, and engaging with industry, which is seen as a key player in the development of new genomic-based clinical interventions. At last, education and training will involve promoting programmes of genomic literacy for health professionals and generally within society, specific training for public health specialists, and development of educational materials, courses, workshops and seminars.

The dynamic and interactive nature of the enterprise is visualised by the doubleheaded arrows. It is informed by societal priorities, generates knowledge as well as using it, and is modulated by the effect of its own outputs and activities. Thus, PHG incorporates a cycle of analysis-strategy-action-evaluation, which describes how the enterprise carries out its activities. 
The wheel of essential Public Health Genomics' activities

Beskow et al. developed genomic-related definitions for the core functions and essential services of public health [10] based on the ten essential public health functions as described first by the Institute of Medicine (IoM) of the US in 1988. This 'blueprint' for integrating genomics into the complete range of public health activities is depicted in Figure 1.2.

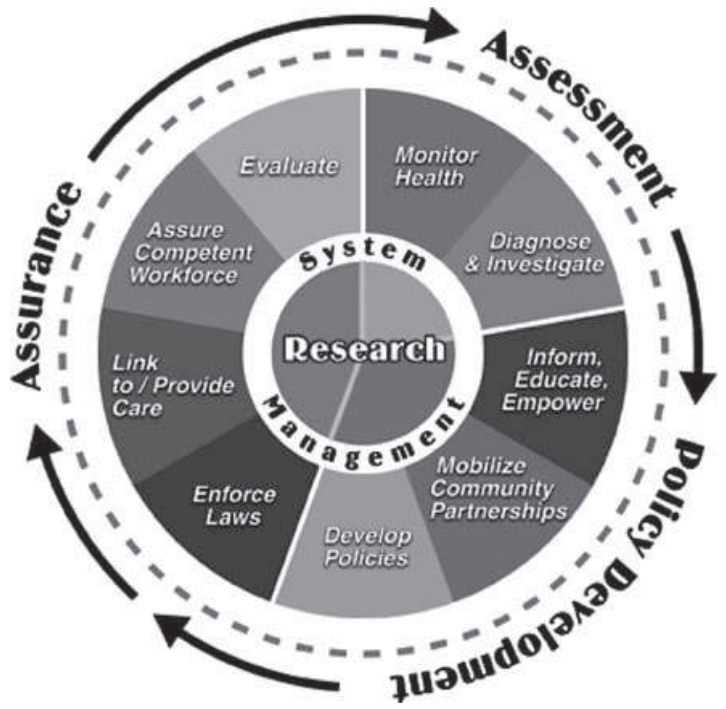

Figure 1.2: The integration of genomics into public health (Adapted from Beskow et al. [10])

The wheel indicates that performance of public health functions and services is neither linear, nor discrete. It is composed of three major parts, which are the core functions of public health ('public health trias'): assessment, policy development, and assurance. Assessment activities provide the knowledge base for policy development including genomics knowledge and for assuring the proper implementation of programs and services that involve genomic components. Policy development activities help identify gaps in scientific knowledge and form the foundation of assuring the effectiveness, accessibility and quality of programs and services. The assurance function, in addition to ensuring that genomics is properly integrated into health-related services, also supplies evaluative information for continuing efforts in assessment and policy development.

Those three core functions are further subdivided in different essential services, which are described in Table 1.1.

In conclusion, the wheel demonstrates the important role for genomics not as a separate specialty but as a fundamental component of existing public health programs and includes also prevention and health promotion programs. 


\section{The European best practice guidelines on PHG, which had been developed by the Public Health Genomics European Network (PHGEN) are based on the public health wheel and the ten essential public health tasks (www. phgen.eu).}

Table 1.1: Essential services for PHG (Adapted from Beskow et al. [10])

\begin{tabular}{|c|c|}
\hline Assessment & $\begin{array}{l}\text { Core function } \\
\text { The regular systematic collection, assembly, analysis and dissemination of information, } \\
\text { including human genome epidemiologic information, on the health of the community. } \\
\text { Related essential services } \\
\text { * Epidemiologic and laboratory research: quantifying the impact of gene variants on } \\
\text { human health and identifying and quantifying the impact on human health of environ- } \\
\text { mental risk factors that interact with gene variants. } \\
\text { * Monitoring health: monitoring health status, including genetic factors, to identify } \\
\text { health problems within the community. } \\
\text { * Diagnosing and investigating: investigating the distribution of genetic and modifiable } \\
\text { risk factors within the community to determine their contribution to identify health } \\
\text { problems and to improve health outcomes. }\end{array}$ \\
\hline Policy development & $\begin{array}{l}\text { Core function } \\
\text { The formulation of standards and guidelines, in collaboration with stakeholders, which } \\
\text { promote the appropriate use of genetic information and the effectiveness, accessibility } \\
\text { and quality of genetic tests and services. } \\
\text { Related essential services } \\
\text { * Policy and communications research: identifying and analysing the economic, social, } \\
\text { ethical and political implications of advances in human genetics, including the informa- } \\
\text { tion and communications needs of stakeholders. } \\
\text { * Informing, educating, empowering, facilitating communication and education about } \\
\text { the integration of genomics into health promotion and disease prevention programs. } \\
\text { * Mobilising partnerships: fostering collaboration between public and private agencies } \\
\text { and constituent groups to promote effective and efficient communication and policy } \\
\text { making about genomics. } \\
\text { * Developing policies: establishing standards and guidelines for when and how genetic } \\
\text { information should be applied to promote health and prevent disease. }\end{array}$ \\
\hline Assurance & $\begin{array}{l}\text { Core function } \\
\text { Assuring constituents that genetic information is used appropriately and that genetic } \\
\text { tests and services meet agreed-upon goals for effectiveness, accessibility and quality. } \\
\text { Related essential services } \\
\text { * Health services research: identifying and analysing the factors that influence the im- } \\
\text { pact of genetic information and the delivery, utilisation and quality of genetic tests and } \\
\text { services. } \\
\text { * Enforcing laws: promoting the enforcement of policies and standards enacted to en- } \\
\text { sure the appropriate use of genetic information and the effectiveness, accessibility and } \\
\text { quality of genetic tests and services. } \\
\text { * Linking to/providing care: ensuring the availability and accessibility of genetic tests } \\
\text { and services and associated interventions to improve health and prevent disease. } \\
\text { * Assuring a competent workforce: ensuring that present and future health profession- } \\
\text { als have training and skills in the appropriate use of genetic information to promote } \\
\text { health and prevent disease. } \\
\text { * Evaluating: evaluating the impact of genetic information and the effectiveness, acces- } \\
\text { sibility and quality of genetic tests and services. }\end{array}$ \\
\hline System management & $\begin{array}{l}\text { Building and maintaining the capacity of the public health infrastructure to integrate } \\
\text { genomics into public health research and practice. }\end{array}$ \\
\hline
\end{tabular}


APPLICATION OF PUBLIC HEALTH GENOMICS ON SMOKING

\section{Introduction}

In European countries, the prevalence of smoking is still around $30 \%$ in the population of 15 years and over [19]. Smoking, which is the single most preventable cause of death, killed around 6 million people worldwide in 2011 with major occurrence in countries of low and middle income. Of these deaths around 5 million are directly due to tobacco use and 600,000 are attributable to second-hand smoking [19].

Smoking is known to be an important risk factor in the incidence of the 4 leading non-communicable disorders (cardiovascular disorders, diabetes, cancers, and chronic respiratory disorders) [19]. It is estimated that smoking is responsible for $71 \%$ of the lung cancer deaths, $42 \%$ chronic respiratory disorders and around $10 \%$ of the cardiovascular disorders. Smoking is not only an important factor in noncommunicable disorders but also in communicable disorders such as tuberculosis or respiratory infections [20]. In 2003, in Germany the total cost of cigarette smoking was about 21.0 billions euros including 7.5 billions of direct costs (cares and drugs) and 13.5 billions euros of indirect costs (mortality and morbidity) [21]. In 2000, the WHO estimated that smoking accounted for $12.3 \%$ of the total years of life lost due to premature mortality and years lived in disability (DALYs) in European regions [22].

Smoking behaviour is a multifactorial trait including both non-genomic and genomic factors. Non-genomic factors are also called environmental. In genetic medicine, environmental factors include all non-inherited factors (e.g. psychological, social or socioeconomic factors). Regarding genomics, twin studies determined that genetic factors influence around 50\% of smoking initiation [23], 75\% of nicotine dependence [24] and around 40 to $60 \%$ of smoking cessation [25]. More specifically, nicotine metabolism and the cascade theory of reward are the two main groups of genes influencing smoking behaviour. Nicotine metabolism indicates how nicotine is absorbed in the organism. The major gene influencing nicotine metabolism is the Cytochrome $\mathrm{P}_{450} 2 \mathrm{~A} 6$ ( $C Y \mathrm{P}_{2} \mathrm{A6}$ ). The cascade theory of reward, which is responsible for the amount of pleasure someone gets when smoking, is based on the action of four different neurotransmitters (serotonin, opioid, gamma-aminobutyric acid (GABA), and dopamine) each containing many genes. (i) Serotoninergic neurons release serotonin (5-HT). This activates (ii) opioidergic neurons that, at the same time, release endogenous opioid. The opioid inhibits the release of (iii) GABA and this inhibition increases the release of (iv) dopamine in the nucleus accumbens (NAcc) in the brain [26]. In a meta-analysis, Munafo et al. reported an association between $D R D_{2}$ Taq1A, a dopamine receptor, and smoking initiation as well as smoking consumption. Moreover, one variant from the serotonin (SLC6A4 5-HTTLPR) and another influencing the nicotine metabolism $\left(C Y_{2} A 6\right)$ were associated with smoking cessation [27]. 
Not only smoking initiation, dependence, and cessation are influenced by genomic factors but also smoking related disorders. Multiple genes have, for example, being associated to chronic obstructive pulmonary disease (COPD) and lung cancer such as CHRNA3, CHRNA5 and CYP1A1 [28]. CHRNA3 and $C H R N A_{5}$ are nicotinic acetylcholine receptors and $C Y P_{1} A_{1}$ is a cytochrome $\mathrm{P}_{450}$ protein. In complex disorders such as smoking, most genomic variables are likely to contribute only to a small part of the phenotype variance. Smoking trajectory from initiation to cessation is made of successive phenotypes as presented on Figure 1.3. For example, the phenotype 'smoking initiation' is assessed by the choice point, never or ever tried cigarette [29].

Therefore, both genomic and environmental factors as their interactions should be taken into account in smoking prevention and cessation. Moreover, cigarette smoking induces, for example, epigenomic and transcriptomic alterations [30, 31]. Methylations are correlated with smoking status and the number of cigarettes per day (e.g. in $F_{2} R_{3}$ and GPR15). However, some DNA methylations seem to be reversible after smoking cessation [31].

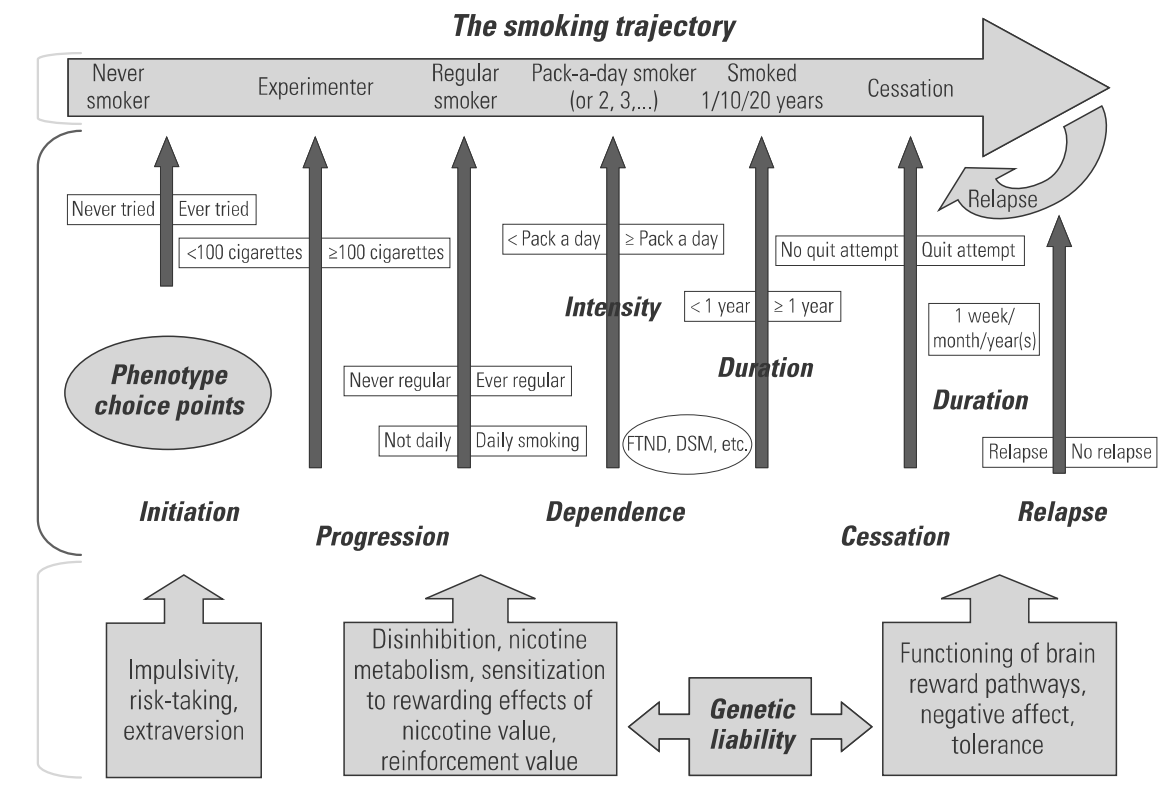

Note. FTND = Fagerström Test for Nicotine Dependence; DSM = Diagnostic and Statistical Manual of Mental Disorders .

Figure 1.3: Phenotype choice points along the smoking trajectory (Adapted from Wanke et al. [29]) 


\section{Smoking behaviour and Public Health Genomics}

In the case of smoking, as in most complex disorders, far less attention has been given to translating genomic information into public health applications in comparison to basic research associating smoking to specific SNPs.

Currently, genomic information is not used in the daily clinical practice as intervention or prevention of smoking. Two main directions of research need further development (i) translation of genomic research on smoking to improve the health of smokers and healthcare interventions, and (ii) to enhance the communication of genome-based risks associated with smoking (initiation, dependence and cessation) and smoking related disorders in the general population.

\section{Translation of genomic research}

Genomic research includes both research on genes and 'omic' features. The translation of genomic research may be conducted through different tools. Firstly and probably one of the most important use is 'employing the power of genetic (and genomic) studies in understanding the underlying biological, behavioural, and environmental factors that will enhance research on etiology, treatment, and prevention for these complex diseases' [32]. Therefore, a better understanding of the biological mechanisms leading to smoking initiation, addiction, cessation, or smoking related disorders is crucial to implement future public health interventions in an effective and efficient way. Indeed, genomic testing has to be used as additional information to environmental factors [33], such as smoking history of the family, history of depression or social support. Considering genomics, environment, and interactions between those two factors is of high importance to improve the motivation and the success in, for example, preventing and quitting smoking and, therefore, on public health outcomes.

In smoking, pharmacogenomics is an approach having high potential. Targeted interventions (in drugs, dose and duration) based on genomic factors can enhance smoking cessation through more precise and personalised interventions [34]. As smoking is a complex disorder, it is unlikely that only one gene will be sufficient to develop personalised interventions [35]. Moreover, the integration of genomebased information, i. e. genome-environmental interactions, into public health policies and practice needs strong evidence of causation, efficacy and cost-effectiveness [35]. Clinical utility and clinical validity are, therefore, of high importance. However, currently there is a lack of evidence supporting both clinical utility (lack of evidence that genomic tests will improve smoking prevention or cessation) and clinical validity (lack of replication in the gene-disorder association studies) [36]. Currently, most personalised interventions in smoking cessation do not include genomic information except in the study of Rose et al., where interactions between nicotine dependence, dose of treatment and quit-success in genotype score were reported. In that study, a score based on 12,058 SNPs indicated interactions with smoking cessation [37]. Prediction models of smoking initiation, addiction, cessation or smoking 
related disorder risks are one specific example of personalised medicine and healthcare. These models could either contain only genomic factors or both genomic and environmental factors, and the latter being more comprehensive of the real problem. To date, few prediction models were developed due partly to the complexity of the mechanisms leading to smoking initiation, addiction, cessation, smoking related disorder risks. However, recently, a model was developed for lung cancer risk in a Chinese population [38]. This prediction model included four different SNPs previously identified in GWAS (rs2736100, rs402710, rs4488809 and rs4083914). However, only moderate discriminatory accuracy was obtained using weighted genetic risk score [38].

\section{Communication of genome-based information}

Communication of genome-based information is necessary for both health professionals and patients. The need is even growing with the availability of direct-toconsumer genetic testing [39]. However, to date, there is an underutilisation of the communication sciences to exchange information on a regular basis [33, 40]. This is why it has been set as one of the priorities for translational research during a 2-day workshop organised by the National Human Genome Research Institute in 2008 [33]. Communication of genome-based information should be used both in health promotion strategies on smoking (e.g. health campaigns) as well as in preventive interventions (e.g. notifying a genomic risk of smoking related disorders in smokers attempting to quit smoking).

The genomic literacy is a relatively new field assessing the knowledge and understanding about genomics in the population. To date few studies assessed the genomic literacy of the general population but due to the specialised and recent topic of genomic, it is suggested to be reasonably low. Therefore, this field needs to be further developed in the near future. In a Japanese study, the term 'genome' appeared unfamiliar with only $14.9 \%$ of the participants answering that they knew the meaning of 'genome'. However, the meaning of the terms 'gene', 'DNA', and 'chromosome' was most widely known [41]. The medium of communication delivery (e. g. television, Internet or information from healthcare providers) may influence the outcome based on the characteristics of the individual. The choice in the type of medium is also susceptible to change over time. The Internet has the potential to become an important tool of communication due to the easiness in sharing and retrieving information and the low-cost in the development of information. Moreover, a very large audience is reached through that kind of media [42]. Moreover, health information on social media, which is a tool dedicated to communication, is suspected to grow dramatically in the next years. Blogs are one example of social media. They allow the spread of information to lay public and health professionals as well as interactions with the public through the commentary's options [42]. However, the most important challenge will be to control the accuracy of the information displayed on such media [33]. To date, most communication-based research on smoking did not take genomics into account except research on genetic risk noti- 
fication of smoking related disorders [43-52]. However, genome-based information is essential to modify health behaviour (e.g. smoking) through different intermediate factors such as emotional factors, self-efficacy or response efficacy [33].

Personalised communication through, for example, genomic notification via webbased communication will not only influence one single individual. Individuals receiving the information will most likely share the information to their circle (e.g. peers) and social network. Therefore, improving genome-based risk communication to a single individual is suggested to have a health impact on the social network of the individual as well $[33,49]$.

\section{AIMS AND OUTLINE OF THE THESIS}

The main objective of the thesis is to describe how genomics contribute to the prevention and the treatment of smoking. Thus, the thesis meets a key task of the $21^{\text {st }}$ century, which is to synthesise and disseminate information [53]. This major objective can be clustered in two parts: (i) to improve healthcare for smokers and (ii) to enhance the communication of genomic risks about smoking in the general population.

The FIRST PART of the thesis focuses on improving the health of smokers and healthcare interventions on smoking. Chapter 2 provides an overview of genomic predictors for smoking initiation and cessation. Chapter 3 presents a literature review including environmental factors, genomic factors and interventions influencing smoking cessation. Based on the literature review, a working model of smoking cessation including all these factors was developed. Moreover, the genetic similarities between tobacco use disorders and other disorders are explored in Chapter 4, based on the observation that genes influencing tobacco use disorders are also associated with many other disorders.

The SECOND PART of the thesis describes the high potential of communicating genome-based information about smoking to the population. The impact of genetic notification of smoking related disease risk on smoking cessation is described in Chapter 5. The availability of information about genetics and smoking on three different social media (YouTube, Facebook and Twitter) is assessed in Chapter 6. Chapter 7 describes the Internet tools and factors influencing the search of information about health, smoking and genetics on the Internet in university students. 


Part I

IMPROVING HEALTHCARE FOR SMOKERS 

GENETIC PREDICTORS FOR SMOKING INITIATION AND CESSATION

Sylviane de Viron

Published as: S. de Viron. (2013) Genetic predictors for smoking initiation and cessation, In: Advanced in smoking cessation. 98-114. DOI: 10.2217/EBO.12.233. 
Preventing smoking initiation and improving smoking cessation has been a major interest of public health for years. Despite this, only $20 \%$ of smokers succeed in quitting smoking with an adequate treatment. Predictors of smoking initiation and cessation have traditionally been focusing on environmental factors such as motivation to quit, age or gender. However, there is more and more evidence that also genetic factors influence smoking initiation and cessation. This chapter provides an overview of the current knowledge on genetic predictors for smoking initiation and cessation. 


\section{INTRODUCTION}

Smoking behaviour (initiation, persistence and cessation) is a complex trait, and depends, just like other behavioural traits and common diseases, on environmental factors, genetic factors and interventions, as well as on the interactions between these different factors (Figure 2.1). For example, not all monozygotic twins have the same smoking status, which demonstrates an impact of environmental factors. The heritable risk was studied in twin studies due to their sharing of genetic factors. In this kind of studies, monozygotic twins are compared to dizygotic twins. Theoretically, monozygotic twins are sharing approximately $100 \%$ of their genes instead of around $50 \%$ for dizygotic twins. Thus, improved correlation of the phenotype might rather be found in monozygotic than in dizygotic twins [54]. Twin studies determined that genetic factors influence around 50\% of smoking initiation [23], 75\% of nicotine dependence [24] and around 40 to $60 \%$ of smoking cessation [25]. The remaining percentage is explained by the shared and non-shared environmental factors. This highlights the high importance of both genetic factors and environmental factors [54].

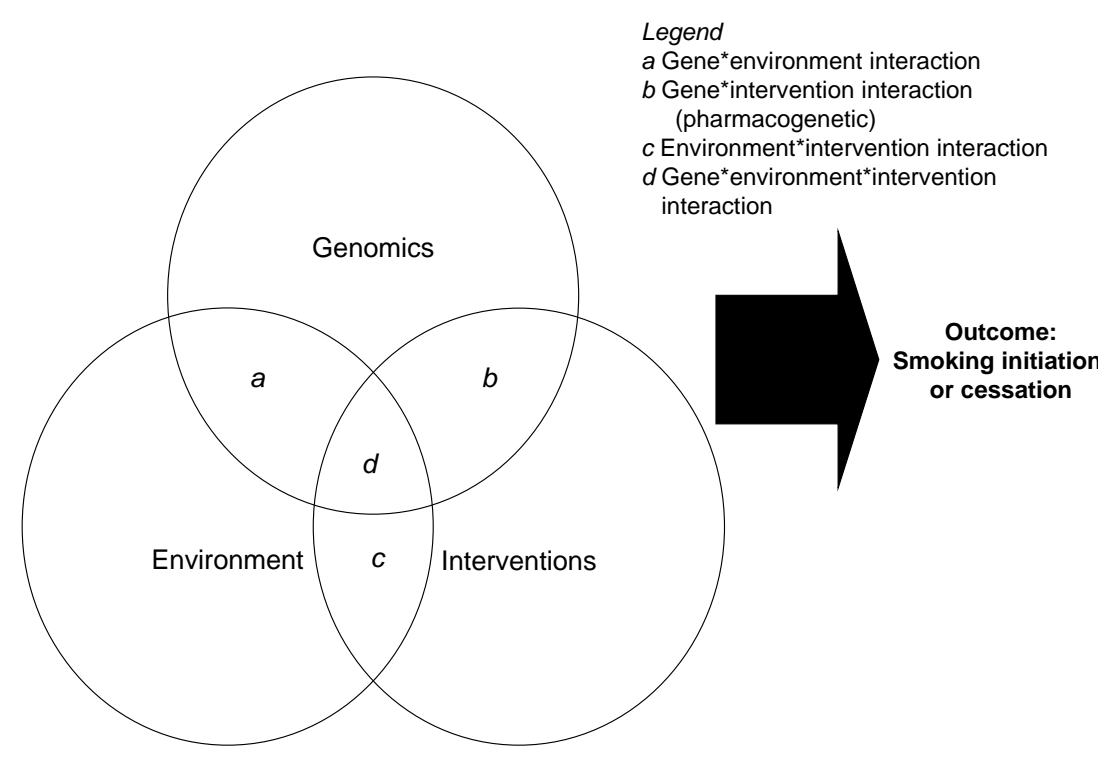

Figure 2.1: Schematic diagram of the multifactorial factors influencing smoking initiation and cessation

Genetic factors are identified through two different ways. First, genetic association studies are looking for potential genetic markers that are based on well established physiologic pathways. Secondly, genome wide association studies (GWAS) are dealing with a large number of single nucleotide polymorphisms (SNPs) across 
the whole genome in a broad sample of participants [55]. This approach is considered as 'hypothesis-free' and consequently, it goes beyond the current knowledge of physiological pathways.

Specific neural pathways are involved in nicotine addiction. Upon inhalation of cigarette smoke, nicotine passes into the bloodstream and, within seconds, crosses the blood-brain barrier to enter the brain. Nicotine binds principally to $\alpha_{4} \beta_{2}$ and $\alpha 7$ nicotinic acetylcholine receptors (nAChRs) located on dopaminergic, glutamatergic and GABAergic neurons in the ventral tegmental area (VTA) of the midbrain, which in turn modulate the release of extracellular dopamine in the nucleus accumbens (NAcc). The activity of dopamine neurons in the VTA is modulated by excitatory glutamatergic and inhibitory GABAergic neurons. The release of dopamine in the nucleus accumbens (NAcc) is responsible for the rewarding and addictive effects of nicotine. Over time, the repeated exposure to nicotine alters the properties of individual neurons and circuits, and this leads to complex behaviours including dependence, tolerance, sensitisation, and craving [56].

\section{GENETIC AND INTERETHNIC DIFFERENCES}

Interethnic differences are of high importance in genetic studies. Some disorders and behaviour occur more frequently in population involving ancestries from the same geographic area. People from the same ethnicity share allelic variations of their genes. If one of these allelic variations is causing a specific disorder or behaviour, the frequency of this particular disorder or behaviour will be more present in that population [57]. Table 2.1 presents an example of the DRD2 C957T, variant in the D2 dopamine receptor. In this example, the frequency of the $\mathrm{C}$ allele of the $D R D_{2} \mathrm{C}_{957} \mathrm{~T}$ variant is about 0.40 in the European population, 0.91 in the African-American and 0.94 in the Asian population (http://wWw.ncbi.nlm.nih.gov/SNP/snp_ref.cgi?rs= rs6277), which could influence the risk of a common disease or the effect of a specific treatment among the different ethnicities. Differences in allele frequencies between ethnicities are found in multiple other genes. Thus, mixing populations could lead to the introduction of confounding in the analyses as the population correlate with both smoking cessation (the outcome) and allele frequency in specific population (the exposure).

Table 2.1: Allele frequency of $D R D 2$ C957T

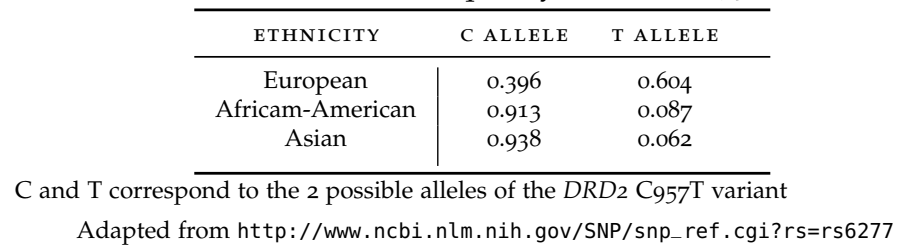


Research on smoking focuses mainly on 2 types of candidate genes (Figure 2.2):

1. Genes influencing the metabolism of nicotine or the pharmacokinetics of nicotine;

2. Genes influencing the reward action induced by nicotine or the pharmacodynamics of nicotine;

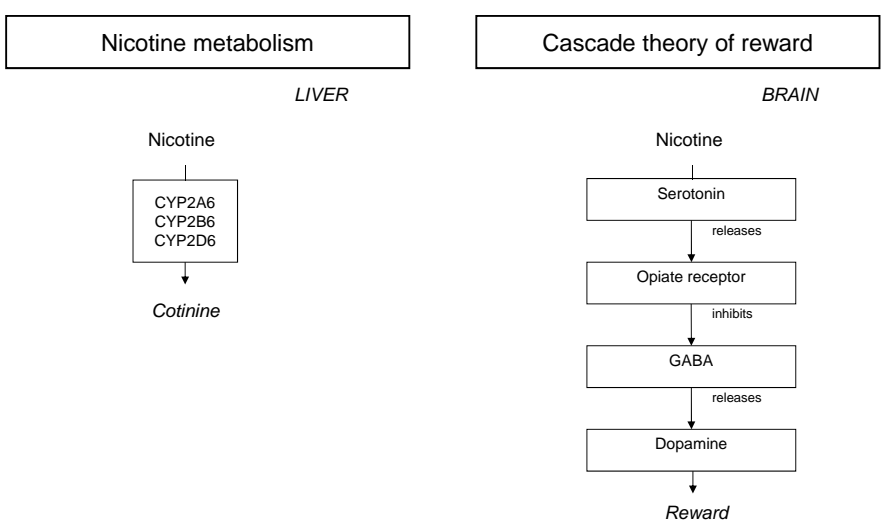

(A) Nicotine metabolism; (B) Cascade theory of reward. CYP, Cytochrome p450

Figure 2.2: Genetic pathways influencing smoking initiation and cessation

\section{Nicotine metabolism}

The major gene influencing the metabolism of nicotine is the hepatic enzyme cytochrome $\mathrm{P}_{450} 2 \mathrm{~A} 6\left(\mathrm{CYP}_{2} \mathrm{~A} 6\right)$ and to a lower proportion cytochrome $\mathrm{P}_{450} 2 \mathrm{~B} 6$ or 2D6. These enzymes mainly metabolise nicotine into cotinine. Cotinine is a wellknown factor that determines the exposure to nicotine [58]. Multiple smoking phenotypes are influenced by $\mathrm{CYP}_{2} A 6$ such as smoking status, nicotine dependence, and smoking cessation [58]. In comparison to normal metabolisers (with $100 \%$ activity), smokers with a genetically poor nicotine metabolism (with $<50 \%$ activity) are often less prone to develop nicotine dependence. Moreover, they may quit smoking more easily possibly due to reduced side effects of smoking abstinence [58]. 


\section{Cascade theory of reward}

'Reward' is defined in the Merriam Webster's dictionary as being a 'stimulus administered to an organism following a correct or desired response that increases the probability of occurrence of the response'. The cascade theory of reward is based on the action of four different neurotransmitters.

- Serotonin;

- Mu-opioid receptor;

- Gamma-aminobutyric acid (GABA);

- Dopamine;

(i) Serotoninergic neurons release serotonin (5-HT). This activates (ii) opioidergic neurons that, at the same time, release endogenous opioid. The opioid inhibits the release of (iii) GABA and this inhibition increases the release of (v) dopamine in the NAcc in the brain [26]. The release of dopamine is responsible for the feeling of pleasure. Smokers with a genetically increased release of dopamine are plausibly more affected by the reward pathway. Consequently, they are more susceptible to become regular smokers when they initiate smoking and are less likely to achieve smoking cessation. These four neurotransmitters have also other functions than the ones mentioned here. The major roles of serotonin are the control of temperature, mood, sleep, locomotion and sensory perception. Studies reported, for example, associations between serotonin genes and depression or addictions (e.g. alcohol, nicotine, gambling or drug). Different treatments are modulating the serotonin release, such as e. g. selective serotonin re-uptake inhibitors [59]. The mu-opioid receptor is the major site of action of opioids (e. g. morphine, heroine or methadone). Studies reported an impact of mu-opioid receptor genes in the reinforcement of learning, in pain response and in addictions [6o]. GABA is a major inhibitory neurotransmitter. Associations with schizophrenia and drug addictions were reported in studies [61]. The main role of dopamine is the control of locomotion and emotion as well as reward. Dopamine release has also an important role in other diseases, such as Parkinson's or Alzheimer's disease [59]. Glutamate and nAChRs also play a role in smoking reward. The glutamate synthesises GABA and plays an important role in training and memory. Glutamate has been related to other diseases such as schizophrenia and epilepsy [62]. Different roles of $n A C h R s$ are defined such as neuronal development, memory, learning, and, of course, reward. Until now, most studies assessing the influence of $\mathrm{nAChRs}$ were based on smoking. However, some studies assessed the changes in nAChRs in different neuropsychiatric diseases (e.g. Alzheimer diseases, Parkinson diseases, or depression). Some treatments are modulating nAChRs such as e. g. selective $\alpha 4 \beta 2$ nicotinic acetylcholine receptor partial agonists [6o]. 
Pharmacogenetics is studying genes influencing the pharmacokinetics and pharmacodynamics of treatments regarding the outcome of interest. Pharmacogenetics is of high interest to improve personalised interventions of smokers regarding their own genotypes by targeting specific drug, dose and duration. Different pharmaceutical interventions are used for smoking cessation, the most important one are the following:

- Nicotine replacement therapies (NRTs);

- Bupropion;

- Varenicline;

Nicotine replacement therapies (NRTs) substitute nicotine and are available in different forms (e.g. patch, spray or gum). As NRTs mimicked nicotine from the cigarette, pharmacogenetic interactions are susceptible with the genetic pathways influencing smoking, thus the pharmacokinetics and the pharmacodynamics of nicotine [63]. Bupropion is an antidepressant used to reduce nicotine withdrawal. Pharmacogenetic interactions with smoking cessation are susceptible as Bupropion inhibits dopamine and norepinephrine reuptake and also influences the action of the serotonin pathway. Moreover, it has also some actions on nAChRs [64]. Varenicline is a partial agonist for $\mathrm{nAChRs}$ (partial for $\alpha_{4} \beta 2$ and full for $\alpha 7 \mathrm{nAChRs).} \mathrm{It} \mathrm{stimu-}$ lates the release of dopamine, thus reduces craving and withdrawal. Pharmacogenetic interactions are thus conceivable regarding the genes influencing nAChRs (e.g. CHRNB2 or $\mathrm{CHRNA}_{4}$ ) and the release of dopamine [65].

PROGRESS IN THE IDENTIFICATION OF GENES INFLUENCING SMOKING INITIATION AND CESSATION

Genes included in Table 2.2 were based on prospective or retrospective studies for smoking initiation and only prospective studies for smoking cessation. Indeed, few studies assessed smoking initiation prospectively. Included studies were based on the general population without mixed background ethnicities for the reasons explained in the 'Genetic and interethnic differences' section. We excluded specific subpopulations such as people mental illness, pregnant women, hospitalised smokers, or referring to another addiction. All candidate genes with at least one significant reported association were selected. Significance was assessed with a p-value lower than 0.05 for the association studies and through the genome-wide significance for GWAS. As in GWAS multiple genetic factors are assessed, correction for multiple testing is important to avoid significant results obtained by chance. A level of significance of $5 \times 10^{-8}$ was proposed (based on the correction of Bonferroni for Imillion SNPs because of the fishing expedition approach). 


\section{Smoking initiation}

For smoking initiation, we considered studies comparing initiators versus non initiators. Only four SNPs demonstrated an association with smoking initiation in the literature. Two SNPs from the serotonin pathway (5-HTTLPR C-759T and 5-HTTLPR G-697T) were associated with smoking initiation in populations of European ancestries [66]. And one SNP influencing the dopamine pathway, CALY (rs2298122), was associated with smoking initiation in an Asian and in a European population [67]. The last association was observed in a GWAS with the BDNF polymorphism (rs6265) [68]). BDNF was demonstrated to modulate the reward pathway, and more specifically the activity of $\alpha 7 \mathrm{nAChRs}$ [69].

\section{Smoking cessation}

Eleven SNPs from 6 different pathways demonstrated an association with smoking cessation and/or an interaction with intervention.

GENES INFLUENCING SMOKING CESSATION. Smoking cessation was reported to be associated with nicotine metabolism (CYP2B6 C1459T) [70], and different neurotransmitters of the reward pathway: the mu-opioid receptor (OPRM1 A118G) [71, 72], different dopamine receptors (DRD2 Taq1A, DRD2-141C, DRD2 C957T, DRD4 VNTR) as well as one enzyme that degrades dopamine (COMT Val $\left.{ }^{108 / 158} \mathrm{Met}\right)\left[73^{-}\right.$ 78] and two different nAChRs (CHRNA3 rs1051730 and CHRNB2 rs2072661) [79-81]. Finally, a GWAS study reported an association of DBH (rs3025343) with smoking cessation [68]. This variant is responsible for the translation of dopamine into norepinephrine.

PHARMACOGENETICS OF SMOKING CESSATION. NRTS interacted with the muopioid receptors (OPRM1 A118G) [72], a dopamine receptor (DRD4 VNTR) [77] and one nAChR (CHRNB2 rs2072661) [81]. As NRTs mimick nicotine from tobacco the different pathways influencing nicotine are susceptible to influence NRTs. However, to our knowledge no studies reported an interaction with genes influencing nicotine metabolism despite its plausible influence. Bupropion interacted with a gene from the nicotine metabolism ( $C Y P_{2} B_{6} 6^{*} 6$ haplotype composed of two loci, CYP2B6 G516T and $\left.A_{78}{ }_{5} G\right)[70,82]$, with a variant of the serotonin neurotransmitter $\left(\mathrm{SLC}_{4} \mathrm{~A}_{4} 5^{-}\right.$ HTTLPR) [83] as well as with two different variants of the dopamine D2 receptors (DRD2 Taq1A [73, 74] and DRD2-141C [76]). Serotonin and dopamine neurotransmitters were highly plausible given their implication in the metabolism of Bupropion. To our knowledge, varenicline has not demonstrated any pharmacogenetic interactions with smoking cessation for the moment. Finally, in an Asian population, an interaction between acupuncture and a polymorphism in the D2 receptor of the dopamine pathway (DRD2 Taq1A) was also reported [75]. This could be in line 
with the suggestion of Yoon et al. that acupuncture could modulate the release of Dopamine [84].

Table 2.2: Candidate genes with associations reported at least once in the literature

\begin{tabular}{|c|c|c|c|c|c|}
\hline SNP & ETHNICITY & $\begin{array}{l}\text { IMPACT } \\
\text { ON SI }\end{array}$ & $\begin{array}{l}\text { IMPACT } \\
\text { ON SC }\end{array}$ & $\begin{array}{c}\text { INTERACTION } \\
\text { WITH INTERVENTION }\end{array}$ & REF. \\
\hline \multicolumn{6}{|l|}{ Nicotine metabolism } \\
\hline$C_{Y P_{2} B 6} \mathrm{C}_{1459} \mathrm{~T}$ & European & No & Yes & No & {$[70]$} \\
\hline $\mathrm{CYP}_{2} \mathrm{~B}^{*} 6\left(\mathrm{G}_{516 \mathrm{~T}}\right.$ and $\left.\mathrm{A}_{78} 8_{5} \mathrm{G}\right)$ & European & No & No & Bupropion & {$[82]$} \\
\hline \multicolumn{6}{|l|}{ Serotonin } \\
\hline 5-HTTLPR C-759T & European & Yes & No & No & {$[66]$} \\
\hline 5-HTTLPR G-697T & European & Yes & No & No & {$[66]$} \\
\hline SLC6 $A_{4}$ 5-HTTLPR & European & No & No & Bupropion & {$[83]$} \\
\hline \multicolumn{6}{|l|}{ Mu-opioid receptor } \\
\hline OPRM1 A118G & European & No & Yes & NRT & {$[71,72]$} \\
\hline \multicolumn{6}{|l|}{ Dopamine } \\
\hline \multirow[t]{2}{*}{$D_{2} D_{2}$ Taq1A } & Asian & No & No & Acupuncture & {$[85]$} \\
\hline & European & No & Yes & Bupropion & {$\left[73^{-75}\right]$} \\
\hline DRD2-141C & European & No & Yes & Bupropion & {$[76]$} \\
\hline $\mathrm{DRD}_{2} \mathrm{C}_{957 \mathrm{~T}}$ & European & No & Yes & No & {$[76]$} \\
\hline $\mathrm{DRD}_{4} \mathrm{VNTR}$ & European & No & Yes & NRT & {$[77]$} \\
\hline & European & No & Yes & No & [78] \\
\hline \multirow{2}{*}{ CALY rs2298122 } & Asian & Yes & No & No & {$[67]$} \\
\hline & European & Yes & No & No & {$[67]$} \\
\hline \multicolumn{6}{|c|}{ Nicotinic acetylcholine receptors (nAChRs) } \\
\hline $\mathrm{CHRNA} 3 \mathrm{rs} 1051730$ & European & No & Yes & No & [79] \\
\hline CHRNB2 rs2072661 & European & No & Yes & NRT & {$[80,81]$} \\
\hline \multicolumn{6}{|l|}{ Other from GWAS } \\
\hline$B D N F$ rs6265 & European & Yes & No & No & {$[68]$} \\
\hline DBH rs3025343 & European & No & Yes & No & {$[68]$} \\
\hline
\end{tabular}

GWAS, Genome-wide association study; NRT, Nicotine replacement therapy; SC, Smoking cessation; SI, Smoking initiation

\section{Gene-gene interactions}

To our knowledge, no gene-gene interactions were reported for smoking initiation. However, in smoking cessation two studies reported gene-gene interactions. In 2006, Dahl et al. demonstrated an interaction between the FREQ gene (rs1054879) and $D R D 2-141 C$ [86]. The FREQ gene has a role in the regulation of $\mathrm{D}_{2}$ receptor signaling. Another study reported a gene-gene interaction between $S L C 6 A_{3}$ and DRD2 Taq1A [87]. Gene-gene interactions might have a key role, as smoking cessation is not a monogenic behaviour. 


\section{CONCLUSION}

Identifying genes influencing smoking initiation and smoking cessation as well as the different interactions (gene-environment, gene-intervention and gene-gene interaction) is still of main importance to understand the mechanism leading to modification of smoking behaviour. For the moment, there is a lack of replication of studies that demonstrate the influence of genetic factors on smoking initiation and cessation in different populations of the same background ethnicity and from different ethnicities, as we observed on Table 2.2. Most studies were based on populations of European ancestries and only two studies explored other ethnic populations (always Asian population). This raises the question on how to activate studies in other parts of the world.

Few studies demonstrated the influence of genetic factors on smoking initiation, probably because smoking can be initiated at any time, which increases the difficulty of testing this, both prospectively and retrospectively. Most studies in this field have focused on the influence of genetic factors on the age of smoking initiation [68]. Moreover, the real starting point of smoking initiation may be strongly influenced by environmental factors. Genetics and gene-environment interactions may then play a central role in the continuation of smoking initiation.

Studies on smoking cessation mainly focused on the neurotransmitter of dopamine, probably because it is the most important molecule leading to the reward or pleasure. From the nicotine metabolism, no studies demonstrated an impact of CYP2A6 on smoking cessation, despite its high implication in the metabolic inactivation of nicotine into cotinine. Especially in European ancestries, this may also be due to the low frequency of poor metabolisers in the studies. Although serotonin is the first neurotransmitter responsible for the reward pathway, no studies were identified in which a significant impact on smoking cessation was found, even though there are several candidate genes (e.g. SLC6A4 5-HTTLPR, TPH1 A779C or HTR1A $\mathrm{C}-1019 \mathrm{G})$. Finally, no associations were reported for GABA or glutamate. Smoking cessation and relapse during smoking cessation are mainly related to nicotine dependence but also to behavioural, gestural and ritualistic components of smoking behaviour. These components may be partly due to genetic factors. However, for the moment, no studies investigated these factors.

For the moment, few studies reported gene-environment, gene-gene interactions that could influence smoking initiation or smoking cessation. Most studies lack the power to detect these kinds of interactions due to an insufficient sample size. The required sample size depends on different factors (e.g. the type of study, the allele frequency or the prevalence of the environmental factor of interest).

Studies observing the genetic predictors of smoking initiation and cessation mostly focused on the nicotine compound of cigarette. However, in Europe around 600 additives are allowed in cigarettes and are susceptible to increase the addiction through, for example, the improvement of the taste or the reduction of the side effects (e.g. irritability) of the cigarette. The principal effects of these additives are 
bronchodilatation, anti-inflammation, analgesia, inhibition of neoplastic cells, antipyretic effect and addiction [88]. Tobacco is also composed of more than 4,000 chemical substances. From these 4,000 chemical substances at least 50 are carcinogenic and 250 noxious. The impact of chemicals on addiction is twofold. First, some chemicals are reinforcing the effect of nicotine (e.g. acetaldehyde). Others are inhibiting enzymes influencing the nicotine metabolism $\left(C_{Y} P_{2} A 6\right)$ (e. g. gammavalerolactone) [89]. Increasing the knowledge of the different compounds influencing smoking initiation, addiction and cessation could improve the comprehension of physiological and genetic pathways influencing smoking initiation, nicotine addiction and smoking cessation.

Genes influencing the nicotine metabolism and the cascade of reward are also associated with different comorbidities and with personality traits of smoking behaviour. As an example for the comorbidities, 3 different neurotransmitters of the reward pathway (serotonin, GABA, and dopamine genes) are also associated with depression (HuGENet). Personality traits are influenced through 4 different neurotransmitters of the reward pathway, serotonin, opioid receptor, GABA and dopamine (HuGENet). Trait theory in psychology is an approach to the study of human personality. Trait theorists are primarily interested in the measurement of traits, which can be defined as habitual patterns of behaviour, thought, and emotion [9o]. According to this perspective, traits are relatively stable over time, differ across individuals and influence behaviour. Five traits are used to describe personality [91, 92]. Proponents of the five-factor approach [91, 92] assume a role of genetics and environment (on personality traits) but offer no explicit causal explanation.

In the future, genetic factors should provide new insights for the prevention of smoking initiation and could also improve interventions and more specifically personalised interventions regarding smoking cessation in tobacco control policies. Informing populations on genetic risk modifying the susceptibility of smoking initiation and cessation as well as smoking related disease risk, could improve the awareness on that problematic. This could both increase the motivation to quit smoking or in the opposite, lead to fatalism and consequently decrease the intention to quit smoking. To improve smoking cessation, genetic factors should be used through different ways:

- Personalised interventions (e. g. more intensive interventions for highly genetically addicted smokers);

- Pharmacogenetic analyses (e.g. targeted interventions in drug, dose and duration depending on alleles of different variants);

- Genetic notification of smoking related disease (e.g. giving genetic notification of smoking related disease risk as a part of the intervention of smoking cessation). Recently, a meta-analysis suggested that genetic notification of cancer risk improved smoking cessation in comparison to control [93];

As demonstrated with the examples above, the most important strengths in using genetic factors for smoking cessation is to improve personalised and individualised 
cares. And at the same time, using genetic factors would enhance global care. Indeed, genetic factors will be only part of the treatment for smoking cessation, other factors such as social support, weight concerns or health status might also be taken into account. Regarding the limitations, genetic factors may just be taken as a part of the factors influencing smoking initiation or smoking cessation. These factors might be studied, as we already mentioned, with environmental factors and interactions. Future studies might also focus on epigenetics and on '-omics'. Epigenetics is the link between genetics and phenotype making gene-environment interactions measurable. In the opposite of genomic factors, it evolves during time. Two main types of epigenetic factors are DNA methylation and histone modification. Most studies observed DNA methylation or histone modification leading to smoking related disease or smoking status (never versus current versus former smokers). However, Wan et al. recently reported that different sites of methylation were associated with current smoking, cigarettes consumption (pack-years) and time since quitting smoking [31]. Different levels of '-omics' are available (Figure 2.3):

- Genomics (referring to DNA);

- Transcriptomics (referring to mRNA);

- Proteomics (referring to proteins);

- Metabolomics (referring to metabolites);

Most researches on '-omics' regarding smoking behaviour are focusing on changes leading to smoking related diseases such as chronic obstructive pulmonary disease (COPD) or lung cancer rather than on the impact on smoking initiation and cessation.

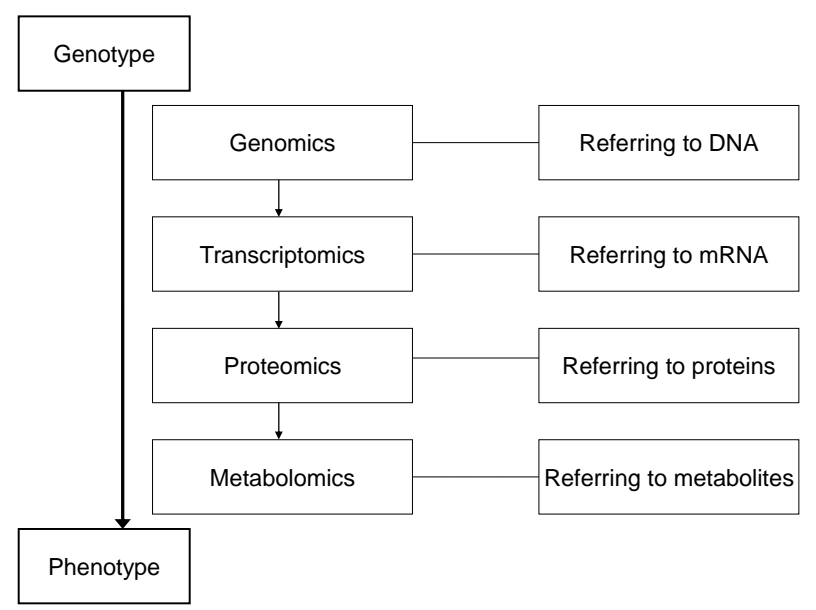

Figure 2.3: Schematic diagram of the hierarchy in the '-omics' 
The genetic impact of smoking initiation and smoking cessation is mainly linked with nicotine

- How genes influence the metabolism of nicotine (e. g. CYP2B6).

- How genes influence the reward feeling caused by nicotine.

\section{Implications for future research}

- Different additives and other chemicals than nicotine are susceptible to influence genetic predictors of smoking initiation and smoking cessation.

- Increased sample sizes are needed to explore gene-gene and gene-environment interactions.

- Investigation of the epigenetics and '-omics' implications in smoking initiation and cessation might give new insights.

\section{Implications of genetic predictors for clinical practice}

- Smoking initiation: Genetic predictors could help in the prevention of smoking initiation.

- Smoking cessation: Genetic predictors could be used for personalised interventions, pharmacogenetic assessment and genetic notification of smoking related disease risk. 

ENVIRONMENTAL AND GENOMIC FACTORS AS WELL AS INTERVENTIONS INFLUENCING SMOKING CESSATION: A SYSTEMATIC REVIEW OF REVIEWS AND A PROPOSED WORKING MODEL

Sylviane de Viron Nuria Malats Johan Van der Heyden Herman Van Oyen Angela Brand

Published as: S. de Viron, N. Malats, J. Van der Heyden, H. Van Oyen, A. Brand. (2013) Environmental and genomic factors as well as interventions influencing smoking cessation: a systematic review of reviews and a proposed working model. Public Health Genomics. 16(4): 159-173. DOI: 10.1159/000351453. 
Background Smoking behaviour is a major public health problem worldwide. Several sources have confirmed the implication of genomic factors in smoking behaviour. These factors interact both with environmental factors and interventions to develop a certain behaviour.

Objectives (1) To describe the environmental factors, the genomic factors as well as the interventions influencing smoking cessation (SC) and (2) to develop a working model incorporating the different factors influencing SC.

Methods Two systematic reviews were conducted using articles in English from the Cochrane library, PubMed and HuGENet from January 2000 to September 2012: (1) a systematic review of systematic reviews and meta-analyses and (2) a systematic review of original research for genomic factors. The proposed working model was developed by making use of previous models of SC and applying iterative process of discussion and re-examination by the authors.

Results We confirmed the importance of the four main factors influencing SC: (1) environmental factors, (2) genomic factors, (3) gene-environment interactions (4) evidence-based interventions. The model demonstrates the complex network of factors influencing SC.

Conclusion The working model of SC proposed a global view of factors influencing SC warranting future research in this area. Future testing of the model will consolidate the understanding of the different factors affecting SC and will help to improve interventions in this field. 


\section{INTRODUCTION}

Smoking is a major public health problem worldwide and the most preventable cause of morbidity and mortality. Five million people die from tobacco consumption every year [94]. Successful smoking cessation (SC) reduces the incidence of comorbidities as well as mortality among smokers [95-97].

Smoking behaviour (smoking initiation, dependence and cessation) is influenced by a high number of both genomic and non-genomic factors. According to twin studies, around 60 to $70 \%$ of the variance of nicotine dependence is explained by inherited factors $[98,99]$. Genomic factors are explored in 3 types of studies: candidate association studies, genome-wide association studies (GWAS) and 'omics' studies (including transcriptomic, proteomic, and metabolomic studies). To assess the correlation of a particular locus with smoking behaviour in candidate association studies, two main hypotheses are used: (1) the cascade theory of reward and (2) the nicotine metabolism. First, based on the 'cascade theory of reward', a number of target genes can be identified that are related to the central behaviour control system. Many different neurophysiological processes are controlled by the modulation of four interlinked compounds: serotonin, opioids, gamma-aminobutyric acid (GABA), and dopamine. In short, serotonin (5-HT) release activates opioids secretion which inhibits the release of GABA. Due to GABA decrease, the release of dopamine is stimulated [10o]. Genetic changes in the pathways controlling the synthesis of these compounds may be involved in the susceptibility to smoking. Second, modifications in genes that influence the 'nicotine metabolism' may also have an impact on smoking behaviour as they define the degradation and the level of nicotine in the body.

Non-genomic factors, also called environmental factors, encompass a broad range of aspects, including cultural, economic, psychological, nutritional, and social factors [101]. SC is reported to be influenced by sociodemographic characteristics, psychological factors and policy decisions [102, 103].

Epigenetic mechanisms, such as methylation, histone modification, miRNAS, and chromatin remodelling are induced by tobacco components. Up to now, epigenetic studies have mostly focused on the development of smoking related disorders, such as lung cancer, chronic obstructive pulmonary disease and cardiovascular disease [104]. However, some methylations appear to be correlated with smoking status and increase with smoking intensity (e.g. RARB and FHIT) [104].

Every year, around $40 \%$ of smokers try to quit smoking for at least one day. Unfortunately, most of them relapse due to nicotine dependence [105, 106]. This high rate of relapse supports the need to further improve care for people who want to stop smoking. In the last decades, plenty of interventions have been implemented in SC (e. g. pharmacotherapy and counselling). However, the rate of relapse after SC is still high. A knowledge synthesis of all factors and interventions influencing SC will give a more global view of this public health problem. A visual overview of the current knowledge, through the development of a conceptual or a working model, 
is particularly relevant. This visual overview illustrates the complex relationships at multiple levels (e.g. psychological, biological and macro-social level) and the relation between the various factors that are involved [107]. Each factor influencing $\mathrm{SC}$ is a single component of a causal mechanism, but a given disorder or trait (e.g. SC) may be the result of more than one causal mechanism. Most causes of SC are neither sufficient nor necessary [108].

The models on SC that were developed up until now had 4 different focuses: (1) application of a general model of behavioural changes on SC [109], (2) impact of tobacco policies [102], (3) social classes [110], and (4) environmental factors and interventions [103, 111]. These models mostly targeted specific populations (e.g. women, adolescents) and environmental factors. Only one model considered genomic factors focusing mainly on pharmacogenetics [111]. However, the conceptual model assessing tobacco use and dependence includes both non-genomic and genomic factors [112]. The introduction of genomic factors led to a more comprehensive and global model of SC and thus improved the knowledge of both providers and smokers.

In this paper, we present the results of a literature review on factors involved in SC. In the last decade, the dramatic increase of the number of molecular analyses at the genetic level has led to recent GWAS and other 'genetic test'-based approaches that could shed a new light on the role of particular factors in SC. The aims of this paper were (i) to review the environmental and the genomic factors as well as the interventions influencing SC reported in the recent literature, and (ii) to develop a working model of SC integrating all relevant factors based on the existing data and the current models.

\section{METHODS}

In a first step, we conducted 2 systematic reviews of factors influencing SC: the first one based on systematic reviews and meta-analyses (the main review), the second one on original research (review on genomic factors). In the latter review, the rationale to focus specifically on genomic factors was based on the hypothesis that most genomic studies were new and, therefore, more often missed out in reviews that were not very recent. Both systematic reviews were used to develop our working model of SC.

\section{Literature review of factors influencing SC}

\section{Outcome}

SC was defined in the Medical Subject Heading (MeSH) index as the discontinuation of the habit of smoking, the inhaling and exhaling of tobacco smoke. This could be self-reported and/or biochemically verified. 


\section{Eligibility criteria}

For the main review, eligible studies consisted of systematic reviews and metaanalyses published in English. The target population included adults from the general population. Studies had to be prospective with a follow-up period of at least 6 months.

The review of genomic factors was restricted to prospective studies in English with a 6-months follow-up period. To avoid confounding, we restricted this review to populations of European ancestries. People from the same ethnicity share allelic variations of their genes. Consequently, frequencies of genetic variants differ among different ethnicities [57].

Articles restricted to a population with a specific disorder (e.g. alcohol addiction or cardiovascular disease) other than smoking were excluded from both reviews. This was also the case for hospitalised patients and pregnant women.

\section{Search strategy (Figure 3.1)}

For the main review, we searched the Cochrane library and PubMed for articles from January 2000 until September 2012 using the term: 'smoking cessation' (PubMed search in Supplementary material - Chapter 3). For the review on genomic factors influencing SC, we searched in PubMed for the same period of time using the terms: 'smoking cessation', 'genetic', 'genomic', 'trancriptomic', 'proteomic', 'metabolomic', 'methylome', and 'epigenome' (PubMed search in Supplementary material - Chapter 3). In addition, we searched for 'smoking cessation' in HuGENet, and we manually reviewed the reference lists of relevant articles and reviews.

One author (SDV) screened the title and electronic abstract identified by the search for relevance and eligibility criteria. Articles that passed this initial screening were further examined.

To make analyses manageable, choices had to be made in the eligibility criteria and the search strategy. Nevertheless, all factors influencing SC were expected to be included in this systematic review, and we presume that the inclusion of other databases would not have contributed to the identification of other factors.

\section{Data extraction}

Factors influencing SC were extracted from reviews and then classified, based on existing models, as follows [102, 103, 109-111]: smoking behaviour, demographic factors, social factors, socioeconomic status (SES), psychological factors, biological factors, health factors, genomic factors, and interventions.

For the interventions, we distinguished three target levels: the individual, the neighbourhood (e.g. household or workplace) and the society (e.g. community or national level). Target levels interact with each other and are at the same time influenced by environmental and genomic factors. 
For the factors identified in the meta-analyses, the overall risk ratio (RR) or odds ratio (OR), the $95 \%$ confidence interval (CI) and the $\mathrm{I}^{2}$ statistics (indicating the level of heterogeneity) were extracted (Table 3.1).

From eligible papers on genomic factors of SC, we extracted information, where available, on the study design, inclusion and exclusion criteria, sample size, interventions, characteristics of participants, outcome length of follow-up, and HardyWeinberg equilibrium (HWE). The HWE assesses the consistency of the genotype frequency of a specific single nucleotide polymorphism (SNP) and other genetic variants (e.g. copy number variation) in the population from generation to generation.

\section{Model formulation}

Building upon the classification of factors extracted from the literature review, we developed the working model of SC (Figure 3.2). The development of this working model was an iterative process based on existing models of SC and discussion and re-examination by the authors.

a.

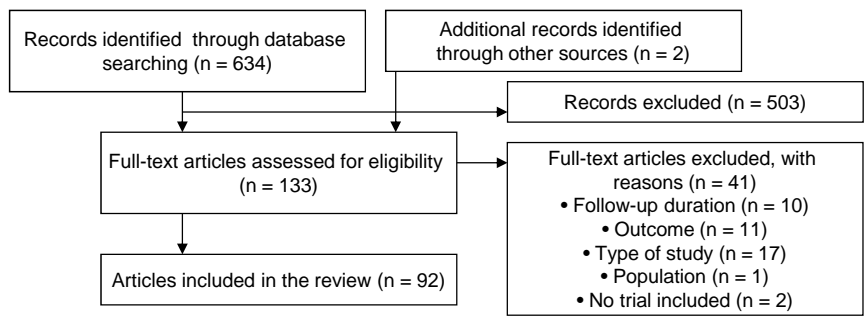

b.

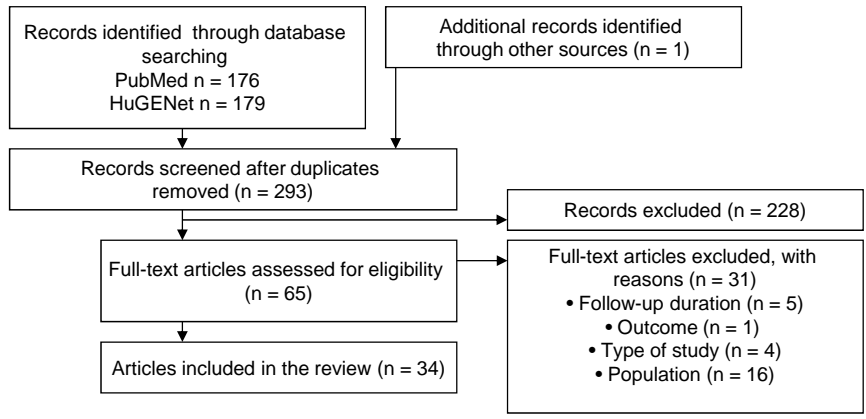

(A) Flow chart of the study selection process of factors influencing SC in reviews, meta-analyses and guidelines. (B)

Flow chart of the study selection process of genomic factors influencing SC in original studies.

Figure 3.1: Flow chart of the study selection process 


\section{Description of studies}

Regarding the main review, among the 636 publications screened in the Cochrane library, PubMed and hand search, 92 met our inclusion criteria (Figure 3.1A.). None of the systematic reviews and meta-analyses discussed genomic factors influencing SC. Actually, meta-analyses mainly assessed the effect of interventions on SC. The overall results (OR, RR and 95\% CI) of the meta-analyses for the different categories of factors are presented in Table 3.1.

Regarding the systematic review of genomic factors influencing SC, out of 293 publications, 34 studies met our inclusion criteria (Figure 3.1B.). Four were based on GWAS, and the other 30 were genetic association studies. None of the selected studies reported an 'omics' association with SC.

Table 3.1: Overall results of the selected meta-analyses

\begin{tabular}{|c|c|c|c|c|}
\hline VARIABLE & $\mathrm{RR} / \mathrm{OR}$ & $95 \%$ CI & FOLLOW-UP & REF. \\
\hline \multicolumn{5}{|l|}{ Environmental factor (Individual level) } \\
\hline Gender (male versus female) & $1.33^{\mathrm{a}}$ & {$[0.91-1.95]$} & $\geqslant 6 \mathrm{~m}$ & [113] \\
\hline Low income (female) & $1.28^{\mathrm{b}}$ & {$[0.96-1.72]$} & $\geqslant 6 \mathrm{~m}$ & [114] \\
\hline Low income (male) & $1.5^{\mathrm{b}}$ & {$[0.96-1.72]$} & $\geqslant 6 \mathrm{~m}$ & [114] \\
\hline Higher cotinine level & $0.80^{a}$ & {$[0.79-3.14]$} & $\geqslant 6 \mathrm{~m}$ & [113] \\
\hline Smoking within 30 minutes after waking up & $0.40^{a}$ & {$[0.63-0.92]$} & $\geqslant 6 \mathrm{~m}$ & [113] \\
\hline Not having other smokings in the household & $1.43^{\mathrm{a}}$ & {$[0.25-0.62]$} & $\geqslant 6 \mathrm{~m}$ & [113] \\
\hline Longest previous quit attempt (female) & $1.23^{\mathrm{a}}$ & {$[1.25-1.65]$} & $\geqslant 6 \mathrm{~m}$ & [113] \\
\hline Longest previous quit attempt (male) & $1.09^{a}$ & {$[0.68-1.02]$} & $\geqslant 6 \mathrm{~m}$ & [113] \\
\hline Male initiating smoking before the age of 16 (versus later age) & $2.10^{\mathrm{a}}$ & {$[1.40-3.00]$} & $\geqslant 6 \mathrm{~m}$ & [113] \\
\hline Smoking reduction before cessation & $2.06^{\mathrm{b}}$ & {$[1.34-3.15]$} & $\geqslant 6 \mathrm{~m}$ & [115] \\
\hline \multicolumn{5}{|l|}{ Pharmacotherapy (Individual level) } \\
\hline \multirow{8}{*}{$\begin{array}{r}\text { NRT over-the-counter } \\
\text { NRT (female) }\end{array}$} & $1.56^{\mathrm{a}}$ & {$[1.16-2.11]$} & $3-6 \mathrm{~m}$ & [116] \\
\hline & $1.94^{\mathrm{a}}$ & {$[1.70-2.20]$} & $6 \mathrm{~m}$ & {$[117]$} \\
\hline & $1.72^{\text {a }}$ & {$[1.61-1.84]$} & $6 \mathrm{~m}$ & [118] \\
\hline & $2.06^{b, c}$ & {$[1.34-3.15]$} & $\geqslant 6 \mathrm{~m}$ & [119] \\
\hline & $1.58^{b}$ & {$[1.50-1.66]$} & $\geqslant 6 \mathrm{~m}$ & [120] \\
\hline & $2.5^{a}$ & {$[1.8-3.6]$} & $2.5-6 \mathrm{~m}$ & [121] \\
\hline & $1.61^{\mathrm{a}}$ & {$[1.29-2.00]$} & $6 \mathrm{~m}$ & [122] \\
\hline & $1.76^{\mathrm{a}}$ & {$[1.48-2.10]$} & $6 \mathrm{~m}$ & {$[117]$} \\
\hline \multirow[t]{2}{*}{ NRT (male) } & $2.20^{\mathrm{a}}$ & {$[1.75-2.76]$} & $6 \mathrm{~m}$ & [122] \\
\hline & $2.16^{a}$ & {$[1.80-2.59]$} & $6 \mathrm{~m}$ & {$[117]$} \\
\hline \multirow[t]{3}{*}{ NRT gum } & $1.71^{\mathrm{a}}$ & {$[1.35-2.21]$} & $6-12 \mathrm{~m}$ & [123] \\
\hline & $1.66^{a}$ & {$[1.52-1.81]$} & 6-12 m & [118] \\
\hline & $1.43^{\mathrm{b}}$ & {$[1.33-1.53]$} & $\geqslant 6 \mathrm{~m}$ & [120] \\
\hline \multirow[t]{2}{*}{ NRT inhaler } & $2.08^{a}$ & {$[1.43-3.04]$} & 6-12 m & [118] \\
\hline & $1.90^{b}$ & {$[1.36-2.67]$} & $\geqslant 6 \mathrm{~m}$ & [120] \\
\hline \multirow[t]{2}{*}{ NRT intranasal spray } & $2.27^{a}$ & {$[1.61-3.20]$} & 6-12 m & [118] \\
\hline & $2.02 \mathrm{~b}$ & {$[1.49-3.73]$} & $\geqslant 6 \mathrm{~m}$ & [120] \\
\hline \multirow[t]{3}{*}{ NRT patch } & $1.74^{\mathrm{a}}$ & {$[1.57-1.93]$} & 6-12 m & [118] \\
\hline & $1.66^{\mathrm{b}}$ & {$[1.53-1.81]$} & $\geqslant 6 \mathrm{~m}$ & [120] \\
\hline & $1.95^{\mathrm{a}}$ & {$[1.65-2.34]$} & 6-12 m & [123] \\
\hline \multirow[t]{2}{*}{ NRT tablet } & $1.73^{\mathrm{a}}$ & {$[1.07-2.80]$} & $6-12 \mathrm{~m}$ & [118] \\
\hline & $2.00^{\mathrm{b}}$ & {$[1.63-2.45]$} & $\geqslant 6 \mathrm{~m}$ & [120] \\
\hline \multirow[t]{2}{*}{ NRT preloading } & $2.17^{a}$ & {$[1.46-3.22]$} & $6 \mathrm{~m}$ & [124] \\
\hline & $1.16^{\mathrm{b}}$ & {$[0.97-1.38]$} & $6-12 \mathrm{~m}$ & [125] \\
\hline & & & \multicolumn{2}{|c|}{ Continued on next page } \\
\hline
\end{tabular}




\begin{tabular}{|c|c|c|c|c|}
\hline VARIABLE & $\mathrm{RR} / \mathrm{OR}$ & $95 \% \mathrm{CI}$ & FOLLOW-UP & REF. \\
\hline Bupropion & $\begin{array}{l}2.07^{a} \\
1.69^{b}\end{array}$ & $\begin{array}{l}{[1.73-2.55]} \\
{[1.53-1.85]}\end{array}$ & $\begin{array}{l}6-12 \mathrm{~m} \\
6-12 \mathrm{~m}\end{array}$ & $\begin{array}{l}{[123]} \\
{[126]}\end{array}$ \\
\hline Nortriptyline & $\begin{array}{l}1.09 \\
2.03\end{array}$ & {$[1.48-2.78]$} & $\geqslant 6 \mathrm{~m}$ & {$\left[\begin{array}{l}{[120]} \\
{[126]}\end{array}\right.$} \\
\hline \multirow[t]{2}{*}{ Varenicline } & $2.41^{\mathrm{a}}$ & {$[1.91-3.12]$} & $\geqslant 6 \mathrm{~m}$ & [123] \\
\hline & $2.27^{b}$ & {$[2.02-2.55]$} & $6-12 \mathrm{~m}$ & {$[65]$} \\
\hline \multirow{2}{*}{ Cytisine } & $1.83^{a}$ & {$[1.12-2.99]$} & $3-6 m$ & [127] \\
\hline & $3.98^{\mathrm{b}}$ & {$[2.01-7.87]$} & $\geqslant 6 \mathrm{~m}$ & {$[65]$} \\
\hline Naltrexone & $1.26^{a}$ & {$[0.80-2.01]$} & $\geqslant 6 \mathrm{~m}$ & [128] \\
\hline \multirow{4}{*}{$\begin{array}{r}\text { Clonidine } \\
\text { Combined therapy }\end{array}$} & $1.89^{a}$ & {$[1.30-2.74]$} & $\geqslant 3 \mathrm{~m}$ & [129] \\
\hline & $2.14^{\mathrm{a}}$ & {$[1.28-3.60]$} & $6-12 \mathrm{~m}$ & [130] \\
\hline & $2.19^{\mathrm{a}}$ & {$[1.7-2.8]$} & $6-12 \mathrm{~m}$ & [131] \\
\hline & $1.54^{\mathrm{b}}$ & {$[1.19-2.00]$} & $6 \mathrm{~m}$ & [132] \\
\hline \multicolumn{5}{|l|}{ Counselling (Individual level) } \\
\hline \multirow[t]{3}{*}{ Individual counselling } & $1.39^{b}$ & {$[1.24-1.57]$} & $\geqslant 5 \mathrm{~m}$ & [133] \\
\hline & $1.20^{\mathrm{a}, \mathrm{c}}$ & {$[0.72-2.00]$} & $6-9 \mathrm{~m}$ & [116] \\
\hline & $1.49^{\mathrm{a}}$ & {$[1.08-2.07]$} & $6-12 \mathrm{~m}$ & [134] \\
\hline \multirow[t]{2}{*}{ Group counselling } & $1.98^{\mathrm{b}}$ & {$[1.60-2.46]$} & $\geqslant 6 \mathrm{~m}$ & [135] \\
\hline & $1.76^{\mathrm{a}}$ & {$[1.11-2.93]$} & $6-12 \mathrm{~m}$ & [134] \\
\hline \multirow{2}{*}{ Telephone counselling } & $1.37^{\mathrm{b}, \mathrm{c}}$ & {$[1.26-1.50]$} & $\geqslant 6 \mathrm{~m}$ & [136] \\
\hline & $1.58^{\mathrm{a}}$ & {$[1.15-2.29]$} & $6-12 \mathrm{~m}$ & [134] \\
\hline Call-back counselling & $1.41^{\mathrm{b}, \mathrm{c}}$ & {$[1.27-1.57]$} & $6-12 \mathrm{~m}$ & [137] \\
\hline \multirow[t]{3}{*}{ Motivational interviewing } & $1.35^{\mathrm{a}}$ & {$[1.02-1.78]$} & $\geqslant 6 \mathrm{~m}$ & [138] \\
\hline & $1.45^{\mathrm{a}}$ & {$[1.14-1.83]$} & $1-12 \mathrm{~m}$ & [139] \\
\hline & $1.70^{b, c}$ & {$[1.32-2.21]$} & $\geqslant 5 \mathrm{~m}$ & [140] \\
\hline \multirow[t]{2}{*}{ Brief advice } & $1.74^{\mathrm{b}, \mathrm{c}}$ & {$[1.37-2.22]$} & $\geqslant 5 \mathrm{~m}$ & [140] \\
\hline & $1.47^{\mathrm{b}}$ & {$[1.24-1.75]$} & $\leqslant 12 \mathrm{~m}$ & [141] \\
\hline Intermediate advice & $1.71^{b, c}$ & {$[1.39-2.09]$} & $\geqslant 5 \mathrm{~m}$ & [140] \\
\hline Intensive advice & $1.60^{b, c}$ & {$[1.13-2.27]$} & $\geqslant 5 \mathrm{~m}$ & [140] \\
\hline \multicolumn{5}{|l|}{ Counselling by provider (Individual level) } \\
\hline \multirow{4}{*}{$\begin{array}{r}\text { Counsellor } \\
\text { Trained professional } \\
\text { Physician }\end{array}$} & $1.82^{b}$ & {$[0.84-3.96]$} & $\geqslant 6 \mathrm{~m}$ & {$[142]$} \\
\hline & $1.60^{a}$ & {$[1.26-2.03]$} & $\geqslant 6 \mathrm{~m}$ & [143] \\
\hline & $1.76^{\mathrm{b}}$ & {$[1.58-1.95]$} & $\geqslant 6 \mathrm{~m}$ & {$[144]$} \\
\hline & $1.87^{\mathrm{b}}$ & {$[1.42-2.45]$} & $\geqslant 6 \mathrm{~m}$ & [142] \\
\hline \multirow[t]{3}{*}{ Nurse } & $1.28^{b, c}$ & {$[1.18-1.38]$} & $\geqslant 6 \mathrm{~m}$ & {$[145]$} \\
\hline & $1.45^{\mathrm{a}, \mathrm{c}}$ & {$[1.17-1.80]$} & $\geqslant 6 \mathrm{~m}$ & [146] \\
\hline & $1.76^{\mathrm{b}}$ & {$[1.21-2.57]$} & $\geqslant 6 \mathrm{~m}$ & [142] \\
\hline \multirow[t]{2}{*}{ Nurse - High intensity } & $1.28 \mathrm{~b}, \mathrm{c}$ & {$[1.18-1.39]$} & $\geqslant 6 \mathrm{~m}$ & [145] \\
\hline & $1.46^{\mathrm{a}, \mathrm{c}}$ & {$[1.12-1.89]$} & $\geqslant 6 \mathrm{~m}$ & [146] \\
\hline \multirow[t]{2}{*}{ Nurse - Low intensity } & $1.27^{\mathrm{b}}$ & {$[0.99-1.62]$} & $\geqslant 6 \mathrm{~m}$ & [145] \\
\hline & $1.43^{\mathrm{a}}$ & {$[0.96-2.14]$} & $\geqslant 6 \mathrm{~m}$ & [146] \\
\hline$[1.04-3.62]$ & $1.94^{\mathrm{b}}$ & {$[1.04-3.62]$} & $\geqslant 6 \mathrm{~m}$ & [142] \\
\hline \multicolumn{5}{|l|}{ Other intervention (Individual level) } \\
\hline Biomedical risk assessment (CO measure + spirometry) & $2.45^{\mathrm{a}}$ & {$[0.73-8.25]$} & $\geqslant 6 \mathrm{~m}$ & [147] \\
\hline \multirow{2}{*}{ CO measure } & $1.07^{\mathrm{a}}$ & {$[0.83-1.39]$} & $\geqslant 6 \mathrm{~m}$ & [147] \\
\hline & $1.06^{\mathrm{b}}$ & {$[0.85-1.32]$} & $6-12 \mathrm{~m}$ & [148] \\
\hline Aversive therapy & $1.98^{\mathrm{a}}$ & {$[1.36-2.90]$} & $\geqslant 6 \mathrm{~m}$ & [149] \\
\hline & $4.26^{a}$ & {$[1.26-14.38]$} & $6-12 \mathrm{~m}$ & {$[150]$} \\
\hline Acupuncture & $3.53^{a, c}$ & {$[1.03-12.07]$} & $6-12 \mathrm{~m}$ & [150] \\
\hline & $1.79^{\mathrm{b}}$ & {$[0.98-3.28]$} & $6-12 \mathrm{~m}$ & [151] \\
\hline Hypnotherapy & $4.55^{\mathrm{a}}$ & {$[0.98-21.01]$} & $6-12 \mathrm{~m}$ & {$[150]$} \\
\hline Web-based intervention & $1.93^{\mathrm{b}}$ & {$[1.44-2.60]$} & $6 \mathrm{~m}$ & [152] \\
\hline & $1.93^{\mathrm{b}}$ & {$[1.27-1.64]$} & 3-12 m & [153] \\
\hline Minimal clinical intervention & $1.50^{\mathrm{a}}$ & {$[0.84-2.78]$} & $6-12 \mathrm{~m}$ & [134] \\
\hline Self-help intervention & $1.28^{\mathrm{b}}$ & {$[0.89-1.82]$} & $\geqslant 5 \mathrm{~m}$ & [142] \\
\hline & $1.08^{b}$ & {$[0.99-1.17]$} & $6-12 \mathrm{~m}$ & [154] \\
\hline Written self-help material & $1.59^{b, c}$ & {$[1.22-2.07]$} & $\geqslant 5 \mathrm{~m}$ & [140] \\
\hline Genetic notification & $1.55^{b}$ & {$[1.06-2.21]$} & $\leqslant 6 \mathrm{~m}$ & [93] \\
\hline & $1.87^{\mathrm{a}}$ & {$[1.20-2.92]$} & $\leqslant 6 \mathrm{~m}$ & [155] \\
\hline
\end{tabular}




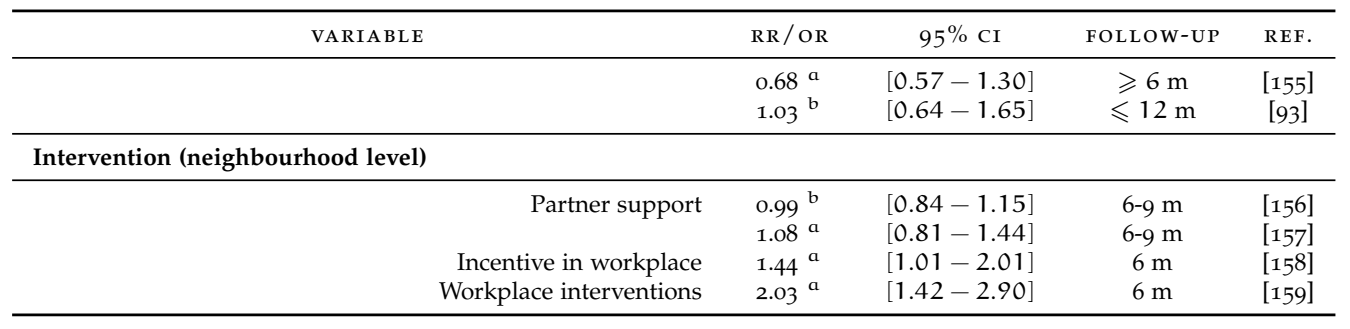

Reference group for the risk ratios and odds ratios were placebo or no intervention; ${ }^{a}$ OR Odds ratio; ${ }^{b}$ RR Risk ratio; ${ }^{c}$ heterogeneity in the analyses $\left(\mathrm{I}^{2} \geqslant 50 \%\right)$

\section{Environmental factor 1: Smoking behaviour}

Factors determining smoking behaviour such as nicotine dependence or past cessation attempts are leading factors influencing the success of SC. Men initiating smoking before the age of 16 have a reduced SC rate in comparison to men starting at a later age $(\mathrm{OR}=2.10, \mathrm{CI}=1.40-3.00)[113,160,161]$. Higher nicotine dependence, indicated for example in those who start smoking within 30 minutes after waking up, reduces SC success [113, 162]. Moreover, a longer duration or a higher number of past cessation attempts is positively associated with SC [113, 162]. Compared to abrupt cessation, smoking reduction doubles the probability of SC $[115,161]$. A positive association with SC is reported for intention-to-quit smoking and the confidence level in quitting [160, 162-164]. Negative beliefs about smoking and health benefit expectancy increase cessation [160-162]. By contrast, a negative impact on SC is reported when enjoying smoking [162] and having easy access to cigarettes [160, 161].

\section{Environmental factor 2: Demographic factors}

Gender does not influence SC (OR $=1.33, \mathrm{CI}=0.91-1.95)$ [113], despite hypotheses that women might have lower SC rates due to, for example, more weight concerns or emotional sensitivity [113, 160, 162]. Regarding age, although in the review of Vangeli et al. [162], 5 studies did not yield significant results, two other studies presented a positive association between age and SC. Gender and age might be moderated by other factors such as e.g. menstrual phase, age at smoking initiation and mortality.

\section{Environmental factor 3: Social factors}

Social factors are especially important due to transmission of social norms between peers. In most studies, being married, couple living together [113, 160, 162], or having children at home [160-162] has no impact on SC. However, living with both biological parents is suggested to enhance SC [160]. Not having friends who smoke 
and the absence of other smokers in the household are positively associated with SC $[113,160,161]$.

\section{Environmental factor 4: Socioeconomic status (SES)}

SES is mainly assessed through education, employment (e. g. employed versus unemployed, blue versus white collar) and income. A lower SES may be associated with reduced SC due to limited information about health concerns, lower access to SC therapy, increased risk of daily stress, and weaker social unacceptability of smoking [103]. However, the relation between SC and the 3 aforementioned socioeconomic factors does not prove to be statistically significant in most studies $[161,162]$. Even a meta-analysis was not able to demonstrate an impact of low income on $\mathrm{SC}$ either in men $(\mathrm{RR}=1.58, \mathrm{CI}=0.79-3.14)$ or in women $(\mathrm{RR}=1.28, \mathrm{CI}=$ 0.96-1.72) [114].

\section{Environmental factor 5: Psychological factors}

Psychological factors such as anxiety (OR 2.2 = for males and 2.6 for females) are likely to reduce SC ability [113]. By contrast, both self-efficacy and intrinsic motivation to quit smoking improve SC $[113,162,165]$. Therefore, mood and stress management programmes are thought to improve SC [166].

\section{Environmental factor 6: Biological factors}

Smoking is known to increase energy expenditure and to reduce appetite. Therefore, a higher weight and more weight concerns may lower SC [166]. Weight concern is linked to psychological and biological factors. A low physical activity is also negatively associated with SC [161]. For women, choosing the target quit day in relation to the menstrual phase, more specifically during the follicular phase, improves SC [166].

\section{Environmental factor 7: Health factors}

Health factors, such as alcohol addiction or depression, may have an important impact on SC due to a reduction of the dopamine release. Indeed, smoking induces the release of dopamine. Alcohol use is negatively associated with SC [113, 161]. A better perceived mental and physical health status enhances SC [160, 161]. However, in most studies there is no significant relation with improved knowledge of health risks associated with smoking [161, 162]. 


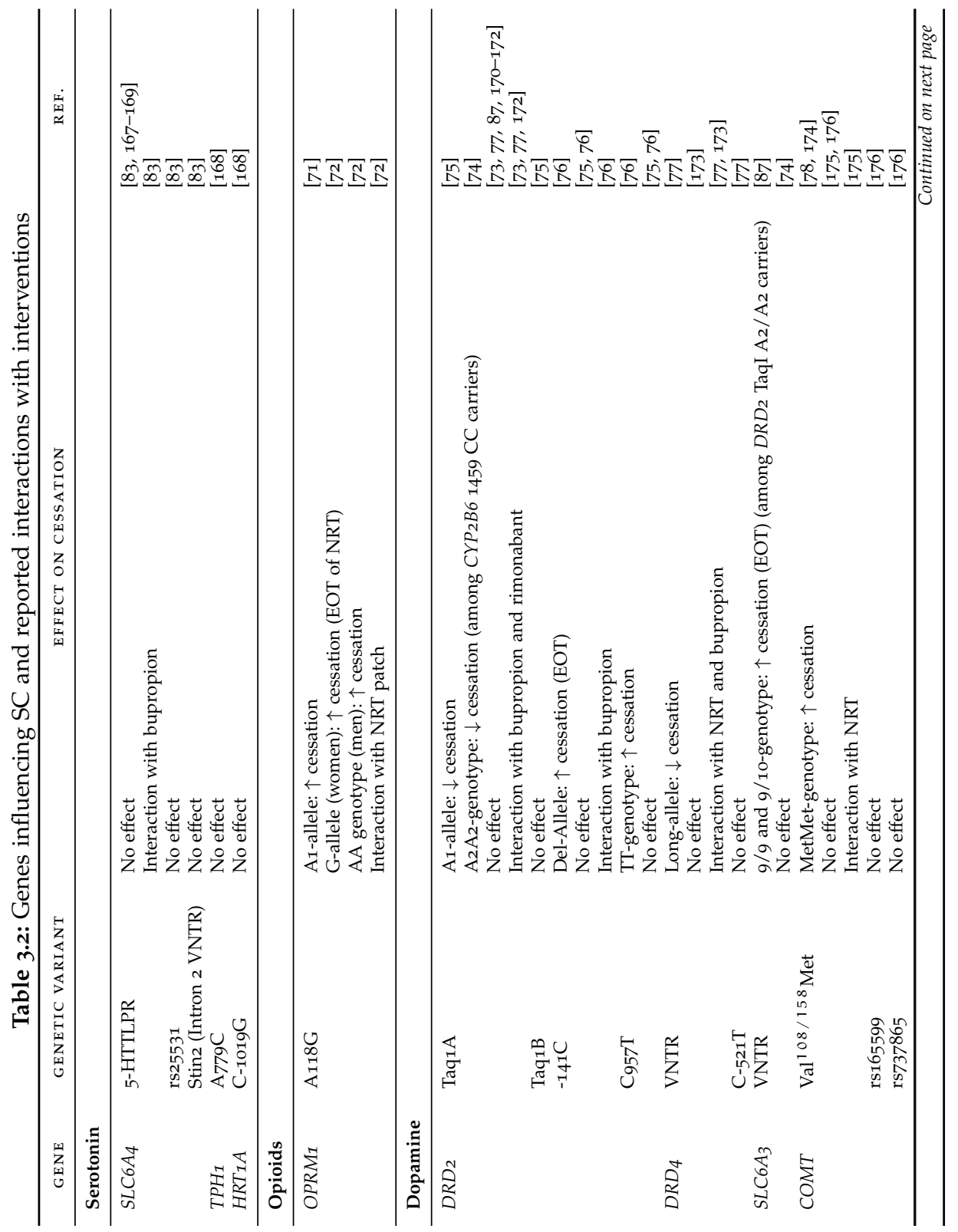




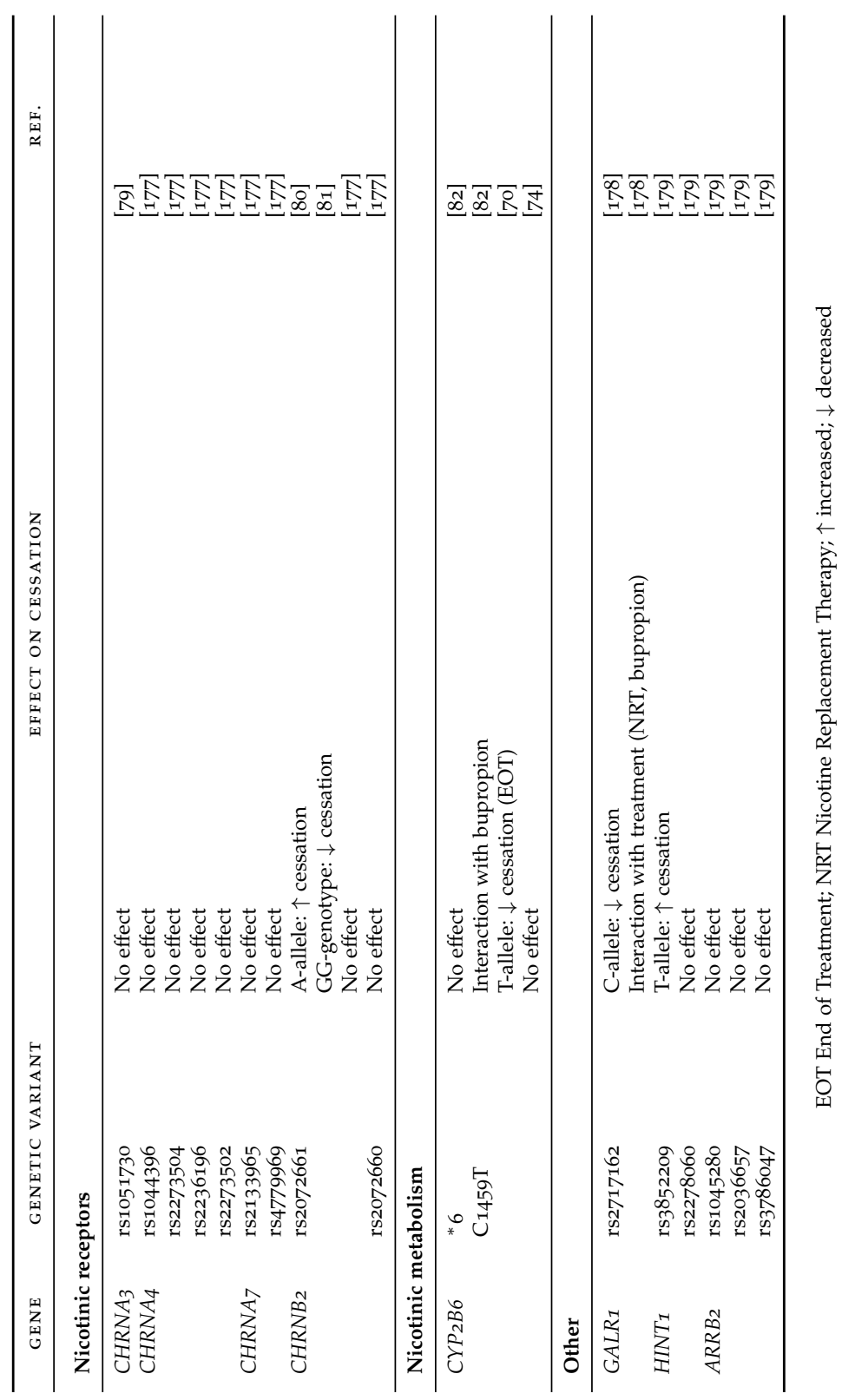


Genomic factors (Table 3.2 and Table 3.3 in Supplementary material - Chapter 3)

Regarding the cascade theory of reward, no study reports a significant impact of serotonin and GABA on SC [83, 167-169]. In opioids, contradictory results are obtained concerning the OPRM1 A118G variant. One study reports that the G-allele improved SC [71]; another confirms this in women but reports an increased SC in men with AA-genotype [72]. Dopamine influences SC through some variants, but some results could not be replicated in different studies $[73,75-77,87,170-$ 173, 175, 176]. The A1-allele of $D R D_{2}$ Taq1A [75] and the long-allele of $D R D_{4}$ VNTR [77] appear to reduce SC. By contrast, the Del-allele of DRD2-141C [76], the TTgenotype of $\mathrm{DRD}_{2} \mathrm{C}_{957 \mathrm{~T}}$ [76], the 9-repeats of $\mathrm{SLC}_{3} \mathrm{~A}_{3} \mathrm{VNTR}$ [87] and the MetMetgenotype of COMT Val ${ }^{108 / 158}$ Met $[78,174]$ improve SC. Gene-gene interactions are reported between $D R D_{2}$ Taq1A-SLC6 33 VNTR [87] and DRD2 Taq1A-CYP2B6 $\mathrm{C}_{1459 \mathrm{~T}}$ [74]. The variant $C H R N B 2$ rs2072661, which encodes for a nicotinic receptor is associated with SC $[80,81]$. However, this finding is not replicated in the study of Spruell et al. [177].

Regarding the nicotine metabolism, only one study reports a significant association with $C Y P_{2} B 6^{*} 6$ in a population of European ancestry [7o].

Different genes that belong neither to the cascade of reward nor to the nicotine metabolism are also associated with SC: the C-allele of GALR1 (rs2717162) decreases SC [178] and the T-allele of HINT1 (rs3852209) increases SC at 6-month follow-up [179].

In none of the GWAS [180-183] a significant association was found (level of significance in GWAS is $\alpha=5 \times 10^{-8}$ ). Also in three (non-GWAS) studies that included many different SNPs, no significant result was found after correction for multiple testing [184-186].

\section{Interventions}

Individual level

At the individual level, two kinds of interventions can be distinguished: (I) pharmacological interventions and (2) non-pharmacological interventions.

Meta-analyses (Table 3.1) indicate that various pharmacological treatments improve SC: nicotine replacement therapy (NRTs), substitutes of nicotine [117-120, 123], Bupropion and nortriptyline (antidepressants) [123, 126, 129, 187-189], and varenicline, cytisine and mecamylamine (agonists of the nicotine acetylcholine receptors) $[65,123,127,190,191]$. Naltrexone, an opioid antagonist, has no significant impact on SC [128]. Cannabinoid type I receptors (rimonabant and taranabant) appear to improve SC at 12-month follow-up, but no result is observed at 6-month follow-up [192].

Different types of non-pharmacological interventions indicate a substantial improvement in SC: individual counselling (although in contrast with 2 other metaanalyses, Coleman et al. [116] did not report any benefit of individual counselling, 
$\mathrm{I}^{2}=68 \%$ ) [116, 133, 134], group counselling [134, 135], web-based interventions [153, 193-195], telephone counselling (with pro-active telephone counselling being even more effective than simple telephone counselling) [134, 136, 137, 196, 197], motivational interviewing [138-140, 198], and genetic notification of smoking related disease risk [93, 155]. Other interventions described in the meta-analyses do not demonstrate statistical significant differences. It concerns: self-help material [142, 154], biomedical risk assessment (e.g. CO or spirometry measurement) [147, 148, 199], incentive-based interventions [158, 193, 200], and hypnosis (even if men were more likely to respond than women) [150, 201, 202]. Positive associations are observed for acupuncture [150, 151], increased physical activity [161, 166, 203, 204] and aversive therapy [149, 150], but the results are not significant. Improvement of SC is also related with the type of provider; e. g. physicians nearly doubled SC in comparison to control [142, 144, 205] and this is also the case for nurses [142, 145, 146], psychologists [142] and trained health professionals [143].

Combining pharmacological and/or non-pharmacological interventions appears to improve SC in comparison to no intervention [130-132] or to monotherapy (RR 1.54, CI 1.19-2.00) [132].

Genetic factors may influence the response to an intervention and, consequently, the success in SC. Some gene-treatment interactions have been reported in European ancestries. Interactions with NRTs are observed for OPRM1 A118G [72], DRD 4 VNTR [77], COMT Val ${ }^{108 / 158}$ Met [175], CHRNB2 rs2072661 [81] and GALR1 rs2717162 [178]. Bupropion interacts with SLC6A4 5-HTTLPR [83], DRD2 TaqIA [73, 74], DRD2-141C [76], DRD4 VNTR [173], CYP2B6*6 [82], and GALR1 rs2717162 [178]. Finally, rimonabant interacts with DRD2 Taq1A [172] (Table 3.2).

Neighbourhood level

At the neighbourhood level, only non-pharmacological interventions are available. Enhancing partner support does not demonstrate any efficacy in improving SC [156, 157]. However, the use of a buddy (when someone is appointed to support a smoker) may be of some benefit in the context of a smokers' clinic [206]. Home smoking restrictions have a positive impact on SC [162]. At workplace, no conclusion can be drawn regarding the efficacy of the interventions, due to their heterogeneity of the interventions [207].

\section{Society level}

Policy-makers launch different actions to reduce smoking prevalence at the society level. Increased taxation [161, 208] and mass media campaigns [161, 208-210] appear to improve SC. However, in lower socioeconomic groups, mass media are more effective when also other interventions are included such as free NRT and telephone counselling or when there is a policy to change the social and structural context of cigarette use [210]. Other interventions such as bans (e.g. banning advertising and 
sponsorship or smoking in public places) [208, 211] and health warnings [208] could help to modify SC through health belief modification.

\section{Smoking cessation working model}

Based on the literature review, we developed a working model of SC consisting of 3 components: (1) factors influencing SC, (2) interventions and (3) SC (Figure 3.2). Each factor in the two first components may influence SC success. Therefore, when attempting to quit smoking all these factors and interventions need to be considered.

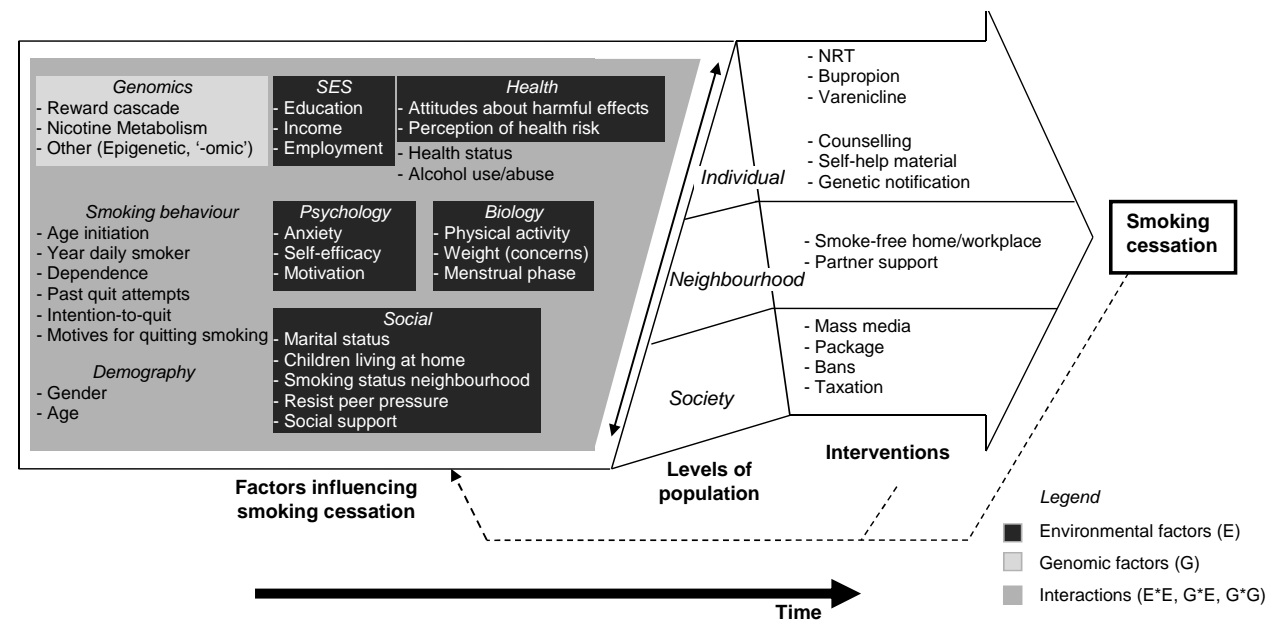

Based on the literature review, we developed a conceptual model of SC consisting of 3 components: (1) factors influencing SC including both genomic and non-genomic factors and interactions (the different factors are interacting as indicated by the light grey background), (2) interventions based on the 3 levels of population (individual, neighbourhood and society level) and, (3) SC (the actual outcome). Interventions are always part of the success of SC. At least unconsciously, interventions at the society level play a role as they are part of our daily life. These 3 components are linked together (indicated by the different arrows) and evolve with time (indicated by the timeline at the bottom of the figure). SES, socioeconomic status; NRT, nicotine replacement therapy.

Figure 3.2: Working model of Smoking Cessation

(1) Factors influencing SC - Eight dimensions are included: smoking behaviour, demography, SES, psychology, social situation, health, biology, and genomics. The eight dimensions cover all factors influencing SC described before, except for the interventions. They constantly evolve and influence one another (indicated by the light grey box covering all factors). For example, smoking induces epigenetic modifications (e.g. methylations) which could influence health [212].

(2) Interventions - Three target levels are defined to classify the interventions: the individual, neighbourhood and society level. Even if smokers do not want to receive specific interventions, some interventions based on the neighbourhood and society level (e.g. smoke-free workplace and taxation) are present in the daily life 
of smokers. Interventions are the most important link between factors influencing SC and SC (represented by the broad arrow).

(3) SC - The actual outcome. SC dynamically interacts with the previous boxes (indicated by the dashed arrows). The success of SC by an individual depends on the different factors influencing SC, neighbourhood and the society in which he lives (e.g. social unacceptability, availability of treatments and bans).

Additionally, the model also takes into consideration the evolution of the environmental and the genomic factors and the interventions over time (represented by the time-line).

\section{DISCUSSION}

To our knowledge, this study is the first to propose a literature review-based working model of SC taking into account environmental factors, genomic factors and interventions. Compared to other models focusing on specific factors such as environmental factors [103] or policy [102], our model is more comprehensive.

Conceptual models are useful for summarising and integrating knowledge. The model demonstrates the importance of research that studies the impact of environmental factors, genomic factors and interventions on SC in the general population in an integrated way. It assumes that SC is a dynamic phenomenon depending on many factors, including interventions at the 3 population levels that we described and environmental factors. For example, a health campaign to stop smoking (intervention) leads to changes in the social acceptability (environmental factor), which could further influence the use of interventions and thus also SC itself.

Our actual model focused on the general population. In a further step, it would be interesting to adapt the model to more specific populations such as smokers with comorbidity or mental illness. The major factors influencing SC will probably be the same, but the effect size might differ. For example, in pregnant women, social pressure will most likely be an important factor. Moreover, for some populations, additional factors appear. In hypertensive smokers, for example, these could be e.g. treatment of hypertension and other cardiovascular risks.

Factors included in the working model of SC were quite similar to the one used in the integrative model of tobacco use and dependence developed by Swan et al. [112] in 2003. In that model, seven dimensions were used to consider tobacco use and dependence: (1) environmental risk factors (e. g. SES and peer smoking), (2) vulnerability factors (e.g. family history of smoking and early smoking experiences), (3) tobacco use trajectory features (e.g. speed of transition to regular use and use of other tobacco substances), (4) motivations (e.g. psychosocial and addictive), (5) nicotine dependence (e.g. the Fageström test for nicotine dependence and nicotine metabolism), (6) nicotine reinforcement (e.g. positive reinforcement and relief from withdrawal), and (7) genetic risk factors in relevant biological pathways (e.g. dopamine and serotonin). Although the seven dimensions used in the integrative model of tobacco use and dependence were classified in a different way than in the 
working model of SC, the included variables were mostly the same. The main difference between the two models is the 'interventions' component. This component is not included in the model of tobacco use and dependence, as dependence does not involve an intervention.

Some factors in the working model could be considered as moderators or confounders (e.g. age could be a confounder in the relation between age at smoking initiation and SC). However, we decided that all factors should be taken into account, as they are part of the causal mechanism leading to SC [108]. A future statistical validation of the model could help to verify this.

A statistical validation of the model is indeed needed. This validation should assess the interactions between the different factors and the size of the effect of each factor. A way to validate this model would be to develop a prospective cohort of smokers that are willing to stop smoking and to observe them for at least 6 months after having stopped. The statistical technique to deal with this is structural equation modelling, in which the number of observations is based on the number of variables. In the proposed working model, around 40 variables are included, which implies that the minimum number of observations should be 820 . The strength of that kind of model would be that it allows evaluating the effect of each single factor and consequently quantifying the effect of the components [108]. Model testing through simulations under different conditions will also help in understanding complex system behaviour in an unexpected fashion [107].

We observe in Table 3.1 that most significant factors influencing SC had a RR or an OR between 1.26 and 2.56. This indicates that none of these factors had a predominant effect on SC, and therefore, all factors may be taken into account when someone wants to quit smoking. Probability helps in the assessment of the contribution of each single component of a causal mechanism. Therefore, it gives a better approximation and more support in public health interventions [213]. However, the interpretation of probabilities often reflects our ignorance about the causal mechanism of a disorder or a trait [213].

Regarding the selection of genomic studies, we focused on European populations because allele frequencies differ among ethnicities. For example, CYP2A6*2 and $\mathrm{CYP}_{2} \mathrm{A6}^{*} 3$ alleles are much more frequent in Asian than in Caucasian populations [214]. Environmental factors are also modified by ethnicity; this is e.g. the case for tobacco use, perception of health risk, or social unacceptability [215]. However, in non-genomic studies, ethnicity is usually not considered as a factor of interest; either mixed ethnicities are used or there is no information on it.

A positive correlation has been reported between the time since quitting and methylation at two

different loci ( $F_{2} R_{3}$ and GPR15), even though no epigenetic study reported an effect on SC. Methylations are found to be rapidly reversible, and therefore, remethylations are suggested after SC [31]. Not only exposure to smoking during childhood and adulthood might influence smoking behaviour. Also exposure to smoking during intra-uterine life is known to induce methylations that increase the risk of disor- 
ders in later life [216]. These methylations might also influence smoking behaviour in a later stage.

The link between psychology and genomic factors influencing SC could be enhanced as the same genomic pathways are implicated. Munafo et al. [217] reported in their meta-analysis that: (i) a genetic variant of the serotonin (5-HTTLPR) was associated with avoidance and aggression traits; (ii) dopamine genes were associated with approach traits $\left(D R D_{3}\right)$ and avoidance traits $\left(D R D_{4}\right)$. This supports the hypothesis that some personalities are more at risk of having problems to quit smoking.

Multiple interactions (gene-gene, gene-environment and environment-environment interactions) influence individual chances to achieve SC. Because of these complex interactions and the association of the same genomic and environmental factors with other disorders, it is still difficult to recognise the influence individual genes have on SC. Even if some studies demonstrated an association of genomic factors with SC, there is still a lack of replication in the literature. Moreover, up to now, only a few studies assessed the multiple interactions (environment-environment, gene-gene and gene-environment interactions) influencing SC. For example, genetobacco control policy interactions have already been suggested in tobacco use. The evolution of tobacco control policies moderates tobacco use by individual genotype [218]. This observation might be applied to SC in the future.

One important limitation of our research is that we restricted the search to prospective studies including 6-month follow-up. This criterion reduced the number of included publications on genomic studies because most original papers and most meta-analyses included retrospective and cross-sectional studies. The same applied for interventions at the society level. For example, even if experts believe that plain packaging will lead to a decline in smoking prevalence [219], currently no systematic review studied the impact of this on SC.

\section{Implications for practice and research}

This review presents various factors influencing SC and provides a framework to further analyse factors influencing SC. Future studies should analyse the interactions and the intensity of the relationship between the different variables of the working model. It will be necessary to validate the model and to assess its quality. Working models are dynamic and constantly changing by the emergence of new evidence. Therefore, the assessment of the quality of this model and the emergence of new developments (e.g. epigenetics, proteomics or vaccines) will lead to modification.

We believe that, in the future, the working model of SC may help to have a global view of factors influencing SC. This will be a key to target interventions on individual smokers and, consequently, improve SC success. The proposed model may also be helpful in the elaboration of future research, aiming to understand the different mechanisms linked to SC. 
SUPPLEMENTARY MATERIAL - CHAPTER 3

PubMed search

PubMed search: Factors influencing smoking cessation

The research on Pubmed was realised using the following code: (smoking cessation [mesh major topic])

Date $27^{\text {th }}$ of September $2012-\mathrm{N}=568$

Filters

- Species: Human

- Languages: English

- Article types: Meta-analysis and Systematic review

- Date of publication: Since January 2000

PubMed search: Genomic factors influencing smoking cessation

The research on Pubmed was realised using the following code:

(smoking cessation[mesh major topic]) and ((genetic) OR (proteomic) OR (transcriptomic) OR (genomic) OR (metabolomic) OR (methylome) OR (epigenome))

Date $27^{\text {th }}$ of September $2012-\mathrm{N}=176$

Filters

- Species: Human

- Languages: English

- Article types: Original papers

- Date of publication: Since January 2000 


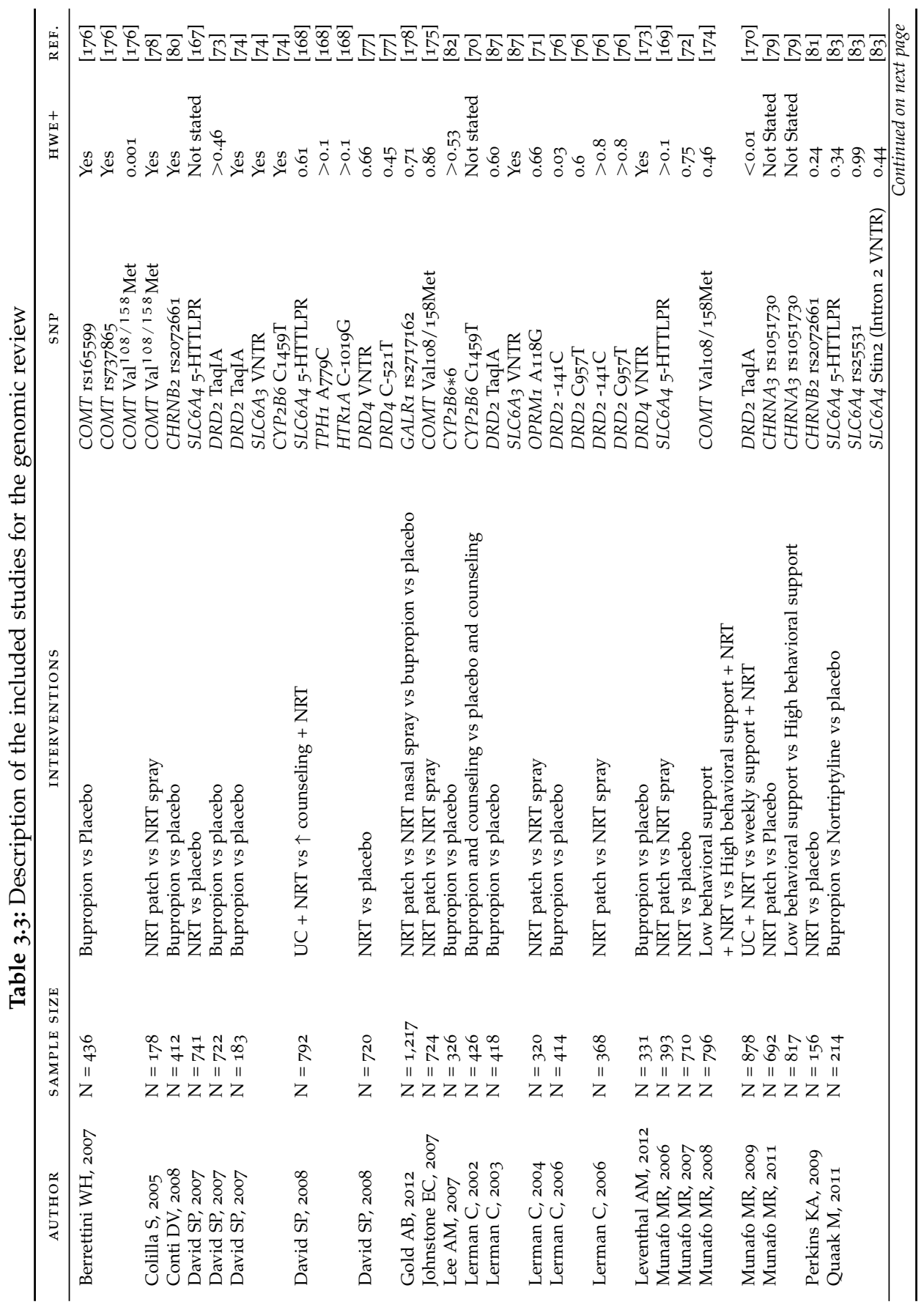




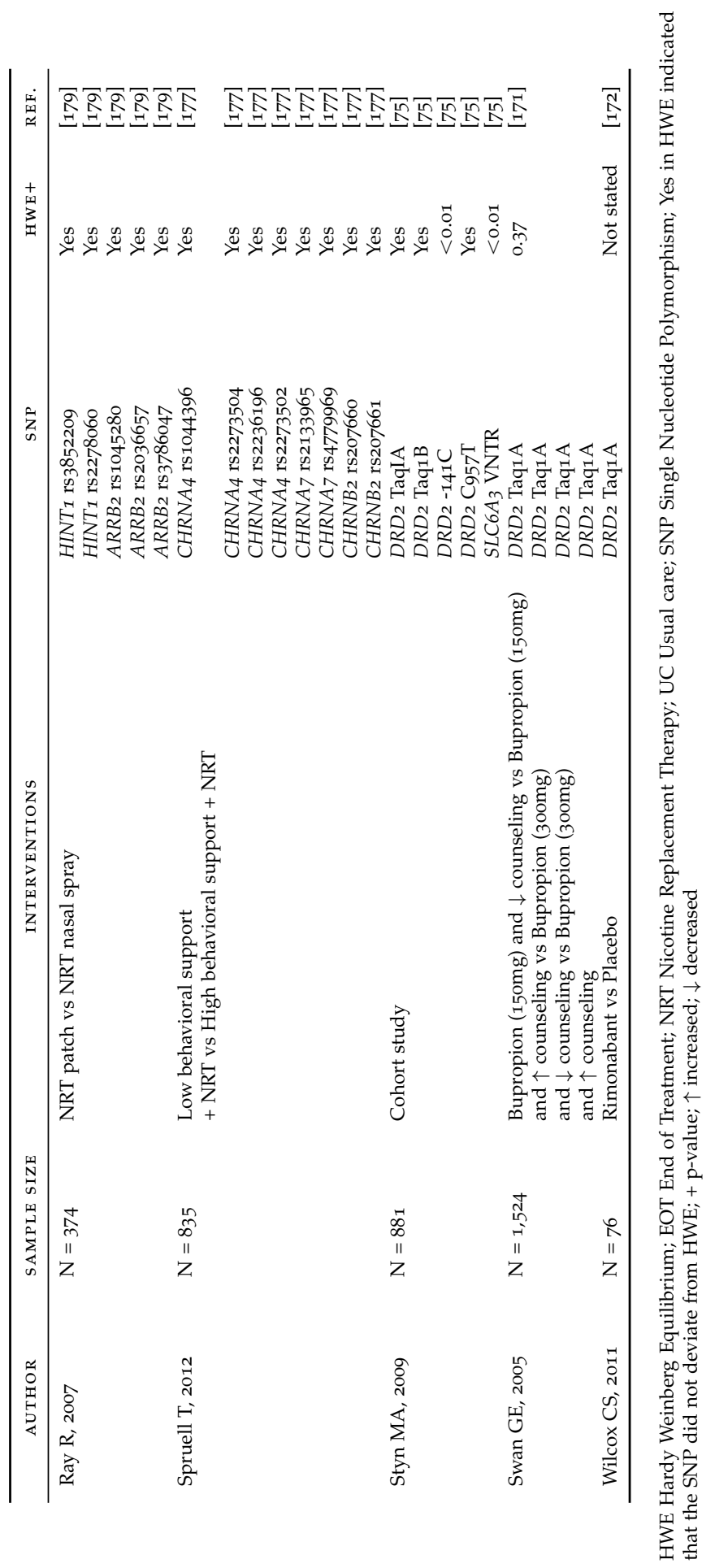



Sylviane de Viron Servaas A. Morré Herman Van Oyen Angela Brand Sander Ouburg 
Background Tobacco use disorder (TUD), as defined by the tobacco use to the detriment of a person's health or social functioning, is associated with various disorders. We hypothesised that mutual variation in genes may partly explain this link.

Objectives The aims of this study were to make a non-exhaustive inventory of the disorders using (partially) the same genetic pathways as TUD, and to describe the genetic similarities between TUD and the selected disorders.

Methods We developed a 3 stage approach : (i) selection of genes influencing TUD using Gene2MeSH and Ingenuity Pathway Analysis (IPA), (ii) selection of disorders associated to the selected genes using IPA and (iii) genetic similarities between disorders associated to TUD using Jaccard distance and cluster analyses.

Results Fourteen disorders and thirty-two genes met our inclusion criteria. The Jaccard distance between pairs of disorders ranged from o.oo (e.g. oesophageal cancer and malignant hypertension) to 0.45 (e.g. bladder cancer and addiction), a lower number Jaccard distance indicating higher similarities between two disorders. Two main clusters of genetically similar disorders were observed, one including coexisting disorders (e.g. addiction and alcoholism) and the other one with sideeffects of smoking (e.g. gastric cancer and malignant hypertension).

Conclusions This exploratory study partly explains the potential genetic components linking TUD to other disorders. Two principle clusters of disorders were observed (i) coexisting disorders of TUD and (ii) side-effects of TUD disorders. Further deepening of this observation in a real life study should allow to strengthen this hypothesis. 


\section{INTRODUCTION}

Tobacco use disorder (TUD) is the greatest cause of preventable death in developed countries and it is a well-known risk factor for many other disorders. As defined in the Medical Subject Heading (MeSH) index [220], TUD is the "Tobacco used to the detriment of a person's health or social functioning. Tobacco dependence is included".

TUD is influenced by multiple environmental as well as genetic factors. Environmental factors in genetic medicine encompass a broad range of factors (e.g. cultural, social, and/or economic factors). The genetic factors belong to two main groups: pathways related to nicotine metabolism and the cascade theory of reward. The most important genes influencing nicotine metabolism are cytochrome $\mathrm{P}_{450} \mathrm{CYP}_{2} A 6$ and $C Y P_{2}$ B6. Genes influencing the cascade theory of reward include the complex network of the serotonin, the opioid, the gamma-aminobutyric acid (GABA), and the dopamine pathways [221]. To assess these gene-environmental interactions is one of the tasks of public health genomics $[6,222]$.

Genes from these two different groups are also influencing other traits and disorders. For example, serotonin genes are associated to personality and psychiatric disorders such as depression [223, 224]. Furthermore, TUD is also related to many traits and disorders. For example, smoking increases the risk of several neoplasms [225] and a higher prevalence of smokers is observed in populations with, for example, schizophrenia [226]. The mechanisms accounting for comorbidity of smoking are, up to now, not well understood.

As proposed by Munafo et al. the relationship between, for example, smoking and depression may be either (i) depression causes people to smoke (through selfmedication of the symptoms), (ii) smoking causes an increased risk of depression (through alterations of neurotransmitter following chronic exposure to tobacco), (iii) bidirectional (acute tobacco smoking reduces negative affect and chronic use increases it), (iv) caused by shared factors such as genetic factors, or (v) the relationship described in i-iii combined with iv. In the latter case the relationship is not causal but due to pleiotropy [227]. This raises questions about the relationship between TUD and disorders: Is it due to causality, pleiotropy, or common pathways in systems medicine? Pleiotropy occurs when a single gene variant influences multiple phenotypic traits. However, systems medicine is a field studying interactions. Thus, it is no more the influence of genetics but genomics such as epigenomics. Answering to that kind of questions is an important step to further improve the treatment and prevention in comorbidities of tobacco use disorder.

Given the high number of disorders associated with TUD, combined with the importance of genetic factors influencing TUD, this study aims to explore the genetic similarities between TUD and disorders genetically associated to TUD. Due to the high number of possible disorders, only an explorative study of disorders with the strongest association with the genes influencing TUD was developed. The aim of this study was (1) to make a non-exhaustive inventory of disorders using the same 
genetic pathway as TUD, (2) to describe the genetic similarities between TUD and the selected disorders.

\section{METHODS}

Figure 4.1 presents the analyses flow of the exploratory study described below.

\section{Candidate gene selection (Figure 4.1A)}

To enhance the robustness of the candidate gene selection, two different tools (Gene2$\mathrm{MeSH}$ and Ingenuity Pathway Analysis) were used.

Gene2MeSH screens all publications on PubMed for genes and MeSH terms and calculates the over-representation of each gene for each specific MeSH term [228]. In June 2012, we searched for genes over-represented in the literature for the following MeSH terms 'tobacco use disorder', 'nicotine', 'smoking' and 'smoking cessation' in human studies of English language. Genes were selected if at least two independent studies reported a significant association with TUD in a European population. Publications were excluded if the entire population had a specific disorder or trait other than nicotine dependence (e.g. alcohol addiction, psychiatric disorder or pregnancy).

In Ingenuity Pathway Analysis (IPA, Ingenuity Systems; www . analys is . ingenuity . com), all genes reported in the search based on the term 'Tobacco use disorder' were selected. IPA allows the development of gene and gene-disease networks through different sources and databases including major NCBI databases (EntrezGene, RefSeq, and OMIM disease associations), microRNA-mRNA target databases, GWAS databases, and Kyoto Encyclopedia of Genes and Genomes (KEGG).

To gain insights into the functional relationships between the selected genes, we developed a network analysis using IPA and STRING 9.o (Search Tool for the Retrieval of Interacting Genes/Proteins) (Figure 4.2) [229]. Genes that were identified by IPA as being related to Tobacco use Disorder (Disease/function search) were selected for pathway analyses. Interactions between the genes were build using the 'Grow' and 'Path Explorer' options filtered for direct interaction, experimentally observed / highly predicted confidence levels, species Homo sapiens, and with the exclusion of chemicals and drugs. Interactions with only one supporting publication were manually excluded as "preliminary". STRING is a web-tool providing genegene and protein-protein association scores based on automatic literature-mining searches. We input our list of genes and set the minimum combined score to 0.900 (highest confidence). The model was built for Homo sapiens. 


\section{Candidate disorder selection (Figure 4.1B)}

Disorders were selected based on their association to the previously selected genes using IPA. As number of associated disorders was expected to be high, we developed two methods of selection. Disorders retrieved from both methods were selected. The first methods included the 20 disorders with the strongest $\mathrm{p}$-value indicating the relationship with the selected genes. In IPA, the p-value refer to a righttailed Fisher's exact tests calculating the likelihood that a set of genes is associated with a specific disorder. The second methods was based on category of disorders and developed in two steps. The first step consisted in selecting the five disorders with the strongest $\mathrm{p}$-value for each category. In the second steps, we limited our disorder selection to the 20 disorders with the strongest $\mathrm{p}$-value. The combination of these two methods allowed the retrieval of broad categories of disorders having the strongest association to TUD.

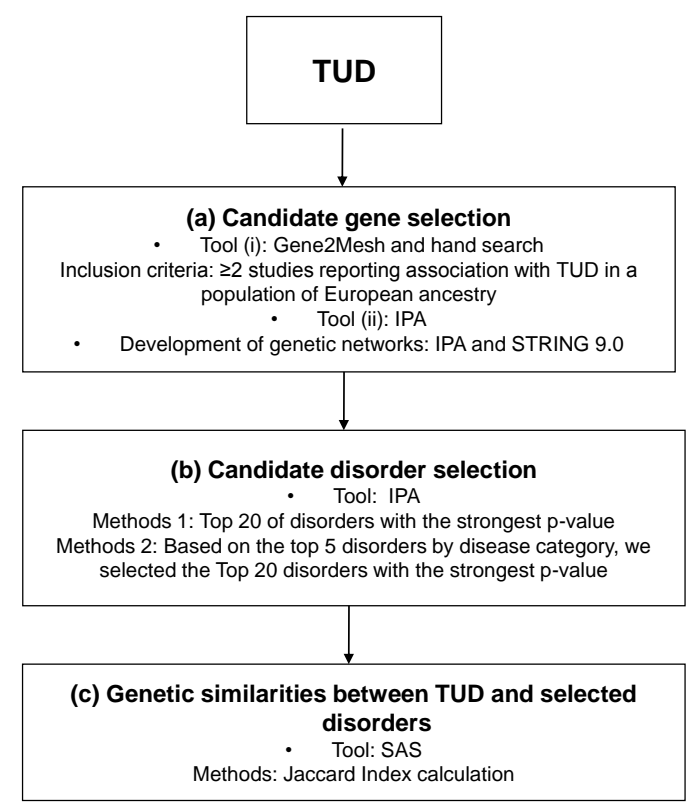

Analysis proceeds from candidate gene selection, to candidate disorder selection, to genetic similarities between TUD and selected disorders. IPA, Ingenuity Pathway Analysis

Figure 4.1: Flow of the analyses 
Genetic similarities between TUD and selected disorders (Figure 4.1C)

Similarity matrix and a cluster analysis of disorders were developed based on genetic variation between disorders using the Jaccard distance [230]. The Jaccard distance measures the dissimilarity between pairs of disorders from a genetic point of view. Each gene is considered as a binary variable that is either present or absent. Therefore, a lower number in the Jaccard distance indicates higher similarities between the two disorders. Cluster analyses were realised using the centroid hierarchical method. The Jaccard distance was estimated using the DISTANCE procedure and the dendrogram was built using the CLUSTER and TREE procedures of SAS 9.2 (SAS Institute, Cary, NC).

Table 4.1: Genes associated with tobacco use disorder selected from Gene2Mesh and Ingenuity

\begin{tabular}{|c|c|c|c|}
\hline GENE & GENE FUNCTION & GENE2MESH & INGENUITY \\
\hline CHAT & Nicotinic receptor & $x$ & \\
\hline CHRNA1 & Nicotinic receptor & & $x$ \\
\hline CHRNA2 & Nicotinic receptor & & $\mathrm{X}$ \\
\hline $\mathrm{CHRNA}_{3}$ & Nicotinic receptor & $x$ & $\mathrm{X}$ \\
\hline $\mathrm{CHRNA}_{4}$ & Nicotinic receptor & $X$ & $x$ \\
\hline CHRNA5 & Nicotinic receptor & $x$ & $x$ \\
\hline CHRNA6 & Nicotinic receptor & & $x$ \\
\hline $\mathrm{CHRNA}_{7}$ & Nicotinic receptor & & $x$ \\
\hline CHRNA9 & Nicotinic receptor & & $X$ \\
\hline CHRNA1O & Nicotinic receptor & & $X$ \\
\hline CHRNB1 & Nicotinic receptor & & $x$ \\
\hline CHRNB2 & Nicotinic receptor & & $x$ \\
\hline $\mathrm{CHRNB}_{3}$ & Nicotinic receptor & & $x$ \\
\hline $\mathrm{CHRNB}_{4}$ & Nicotinic receptor & & $X$ \\
\hline CHRND & Nicotinic receptor & & $\mathrm{X}$ \\
\hline CHRNE & Nicotinic receptor & & $\mathrm{X}$ \\
\hline CHRNG & Nicotinic receptor & & $x$ \\
\hline COMT & Dopamine & $X$ & \\
\hline$C Y P_{2} A 6$ & Nicotine metabolism & $X$ & \\
\hline$D D C$ & Dopamine & $x$ & $x$ \\
\hline $\mathrm{DRD}_{2}$ & Dopamine & $X$ & \\
\hline$G A B B R 2$ & GABA & & $x$ \\
\hline$G A B R A 2$ & GABA & $X$ & \\
\hline $\mathrm{GABR}_{4}$ & GABA & $X$ & \\
\hline NRXN1 & Neurexin & & $x$ \\
\hline$O P R D_{1}$ & Opioid receptor & & $X$ \\
\hline OPRM1 & Opioid receptor & & $x$ \\
\hline$O P R K_{1}$ & Opioid receptor & & $X$ \\
\hline SIGMAR1 & Non-opioid receptor & & $X$ \\
\hline $\mathrm{SLC}_{6} \mathrm{~A}_{3}$ & Dopamine & $x$ & $X$ \\
\hline $\mathrm{SLC}_{6} \mathrm{~A}_{4}$ & Serotonin & $x$ & $X$ \\
\hline TPH1 & Serotonin & $X$ & \\
\hline
\end{tabular}

Selected genes are represented with the $\mathrm{X}$ in the boxes; GABA, Gamma-aminobutyric acid 
RESULTS

\section{Candidate gene selection}

Thirty-two genes were selected, among them 26 were retrieved from Ingenuity and 12 from Gene2Mesh (Table 4.1). Seventeen of them were nicotinic receptors (CHAT, CHRNA1, CHRNA10, CHRNA2, CHRNA3, CHRNA4, CHRNA5, CHRNA6, CHRNA7, CHRNA9, CHRNB1, CHRNB2, CHRNB3, CHRNB4, CHRND, CHRNE, and CHRNG). One gene influenced nicotine metabolism (CYP2A6).

From the cascade of reward, 2 serotoninergic genes (SLC6A4 and TPH1), 3 opioid receptors (OPRM1, OPRD1, and OPRK1), 3 GABA (GABBR2, GABRA2, and $\left.G A B R A_{4}\right)$ and 4 dopaminergic genes (COMT, DDC, DRD2, and SLC6A3) were associated to TUD. Two other genes belonging neither to the nicotine metabolism nor the cascade theory of reward were selected (NRXN1 and SIGMAR1). NRXN1 encodes for a synaptic neuronal adhesion molecule and SIGMARI is implicated in cellular differentiation, neuroplasticity, neuroprotection and cognitive functioning of the brain.

Figure 4.2 presented the smallest network hypothesised by either IPA or STRING based on the 32 selected genes. In IPA, two main groups of genes were obtained including both nicotinic receptors. However, most genes, such as those influencing the cascade theory of reward, were not interconnected (Figure 4.2A). In STRING (Figure 4.2B) with a confidence level of 0.900 , two main groups of genes appeared to be connected. The first one includes mainly genes from the cascade theory of reward (DDC, TPH1, SLC6 $44, C O M T, D R D_{2}, O P R M_{1}, O P K_{1}, O P R D_{1}, S L C 6 A_{3}$, and $\left.S I G M A R_{1}\right)$ and the second includes nicotinic receptors (CHAT, CHRNA2, CHRNA3, $\mathrm{CHRNA}_{4}, \mathrm{CHRNA}_{5}, \mathrm{CHRNA}_{7}, \mathrm{CHRNB}_{2}$, and $\mathrm{CHRNB}_{4}$ ).

\section{Candidate disorder selection}

Of the 20 disorders selected from both methods, 14 were in common (Table 4.2). These disorders covered a broad range of categories including substance related disorders (addiction and alcoholism), psychiatric disorders (depressive disorders, schizophrenia, and schizoaffective disorders), cancer (oesophageal cancer, gastric cancer, and bladder cancer), cardiovascular disorders (stroke, coronary disease, vascular disorder, and malignant hypertension) and psychomotor disorders (motor dysfunction and psychomotor agitation).

\section{Genetic similarities between TUD and selected disorders}

The Jaccard distance ranged from o.oo (bladder cancer-malignant hypertension, oesophageal cancer-bladder cancer, oesophageal cancer-malignant hypertension, and alcoholism-schizoaffective disorder) to 0.45 (addiction-bladder cancer, addictionmalignant hypertension, and addiction-oesophageal cancer) (Table 4.3). With a Jac- 

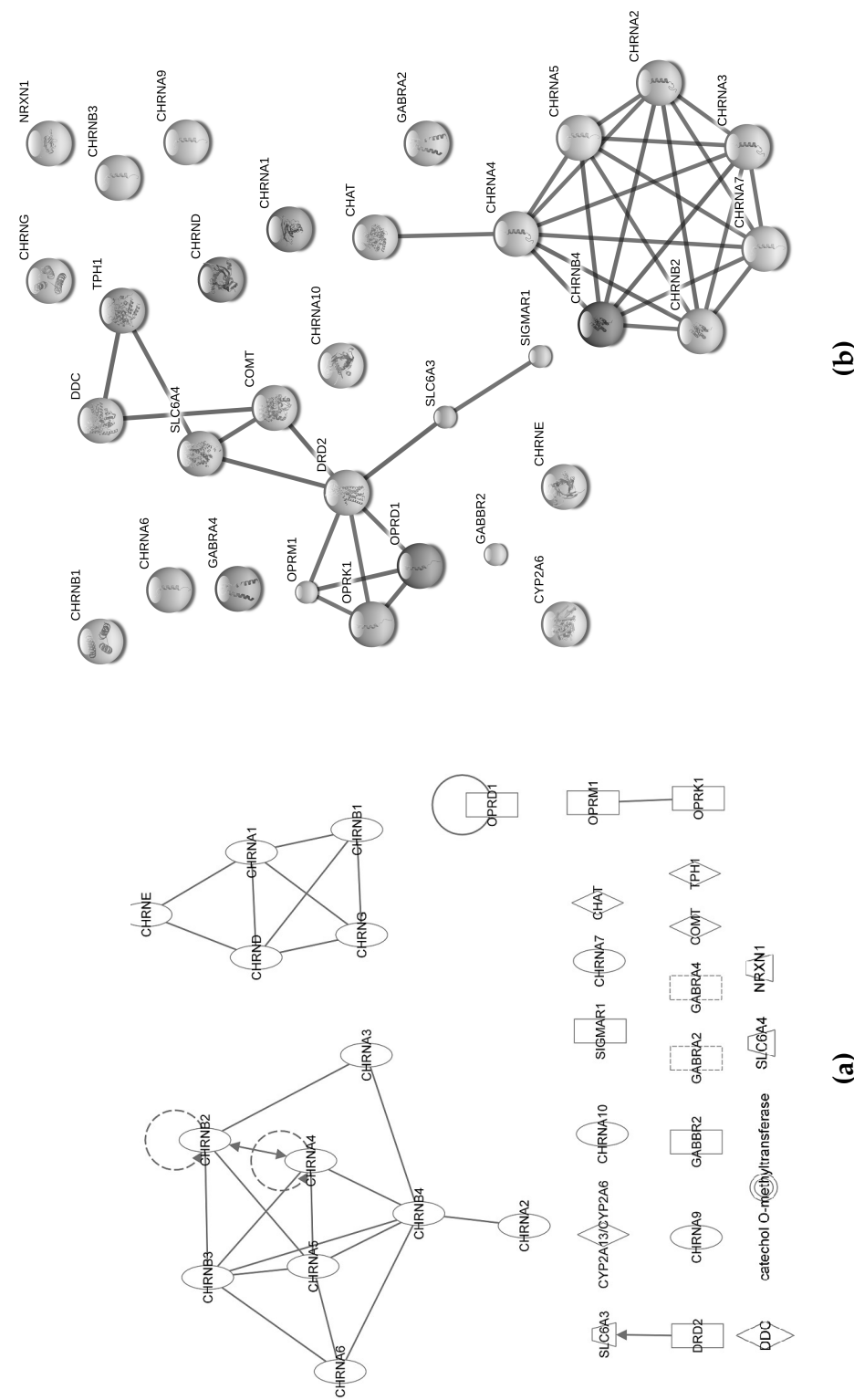

త

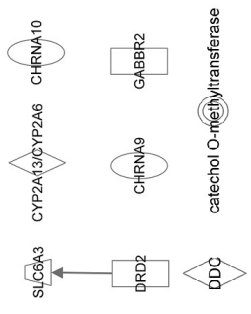

(a) Genetic network obtained from Ingenuity Pathway Analysis. The different shapes of nodes represent the functional class of the gene product. Edges with dashed lines show indirect interaction, while a continuous line represents direct interactions; (b) Genetic network obtained from STRING network. The STRING network incorporates the interactions of the selected genes with the highest confidence level (level of 0.900 ). Stronger associations are represented by thicker lines.

Figure 4.2: Genetic network analyses using Ingenuity Pathway Analysis and STRING 
card distance of 0.00 , four pairs of disorders were exactly similar from a genetic point of view. The dendrogram obtained from the cluster analysis in Figure 4.3 presented two main clusters of disorders. The first cluster contained disorders that are mostly side effects of smoking (e.g. vascular disorder, gastric cancer, and malignant hypertension) [231] and the second cluster disorders that are coexisting to smoking (e. g. addiction, alcoholism, and depressive disorders) [232].

Table 4.2: Disorders associated with genes influencing tobacco use disorder selected from methods 1 and 2

\begin{tabular}{|c|c|c|}
\hline DISORDER & METHODS 1 & METHODS 2 \\
\hline Addiction & $x$ & $x$ \\
\hline Alcoholism & $\mathrm{x}$ & $x$ \\
\hline Bladder cancer & & $x$ \\
\hline Cervical cancer & & $x$ \\
\hline Coronary disease & $\mathrm{X}$ & $\mathrm{X}$ \\
\hline Delirium & $\mathrm{X}$ & \\
\hline Depressive disorder & $\mathrm{X}$ & $\mathrm{X}$ \\
\hline Dyskinesia & $\mathrm{X}$ & \\
\hline Gastric cancer & $\mathrm{X}$ & $x$ \\
\hline Gastrointestinal tract cancer & & $\mathrm{X}$ \\
\hline Hypertension & & $\mathrm{X}$ \\
\hline Insomnia & $\mathrm{X}$ & \\
\hline Leukaemia & & $\mathrm{X}$ \\
\hline Liver cancer & & $x$ \\
\hline Malignant hypertension & $\mathrm{X}$ & $\mathrm{X}$ \\
\hline Mood disorders & $\mathrm{X}$ & \\
\hline Movement disorders & $\mathrm{X}$ & \\
\hline Motor dysfunction & $\mathrm{X}$ & $\mathrm{X}$ \\
\hline Oesophageal cancer & $\mathrm{X}$ & $\mathrm{X}$ \\
\hline Pancreatic cancer & & $\mathrm{X}$ \\
\hline Psychomotor agitation & $X$ & $\mathrm{X}$ \\
\hline Schizophrenia & $\mathrm{X}$ & $\mathrm{X}$ \\
\hline Schizoaffective disorder & $\mathrm{X}$ & $\mathrm{X}$ \\
\hline Stroke & $\mathrm{X}$ & $\mathrm{X}$ \\
\hline Subarachnoid haemorrhage & $\mathrm{x}$ & \\
\hline Vascular disorder & $\mathrm{X}$ & $\mathrm{X}$ \\
\hline
\end{tabular}

Methods 1, 20 disorders with the strongest $p$-value indicating the relationship with the selected genes using Ingenuity Pathway Analysis; Method 2, based on category of disorders and developed in two steps: Firstly selection of the five disorders with the strongest p-value for each category and secondly limitation to the 20 disorders with the strongest p-value; Final selected disorders are the one retrieved from both methods 1 and methods 2.

\section{DISCUSSION}

This exploratory study is a first step towards evaluating the genetic similarities between TUD and disorders genetically associated to TUD. Genes influencing TUD were associated with variety of other disorders. Cluster analyses reported two main clusters of disorders. The first cluster included disorders that are side effects of smoking (including e.g. vascular disorder, gastric cancer, and malignant hypertension) while the second cluster included coexisting disorders of smoking (including e. g. addiction, alcoholism, and depressive disorder). 


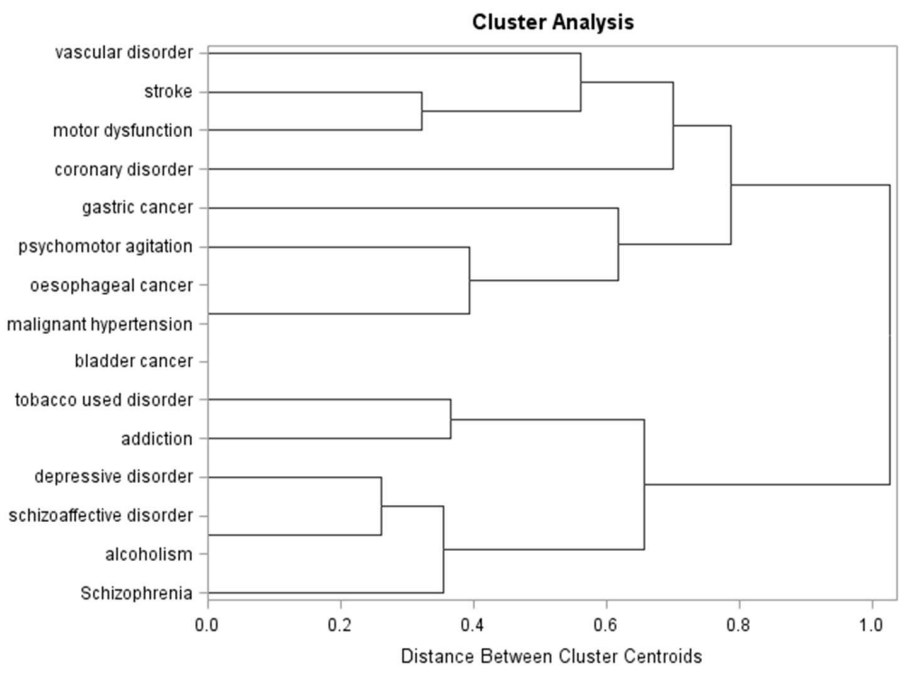

Figure 4.3: Dendrogram of disorders associated with the same genes as TUD resulting from centroid hierarchical cluster analysis, using the Jaccard distance obtained from 32 genes

To our knowledge, this study is the first to show that literature review combined with gene pathway analysis can provide relevant information in understanding the genetic basis for similarities between TUD and associated disorders; even if genes are not the only factors linking TUD to other disorders. Among other factors, environmental factors, treatments, and epigenetic modifications mediate the relation between TUD and other disorders.

The prevalence of coexisting disorders of smoking including mental health and addictive disorders appear to be 2 to 4 times higher in smokers than in the general population. Different theories were developed to explain this higher prevalence: (i) intrinsic factors such as genes that predispose people with mental and addictive disorders to smoke, (ii) self-treatment of mental and addictive disorders through TUD, (iii) common social and environmental determinants for TUD, and (iv) modulation of neurotransmitters involved in the development of mental health and addictive disorders due to TUD [233]. Shared genetic variation of $C H R N A 7$ is, for example, reported in TUD and schizophrenia [233]. Regarding disorders that are side-effects of smoking, the most plausible theory is epigenomic changes due to carcinogenic compounds of tobacco [104]. For example, it was reported that smoking induced a down-regulation of the interferon IFIT1 involved in the progression and invasiveness of bladder cancer [30]. Therefore, genes are just one track to link TUD and related disorders. 
GENETIC SIMILARITIES BETWEEN COMORBIDITIES OF TOBACCO USE DISORDERS $\mid 67$

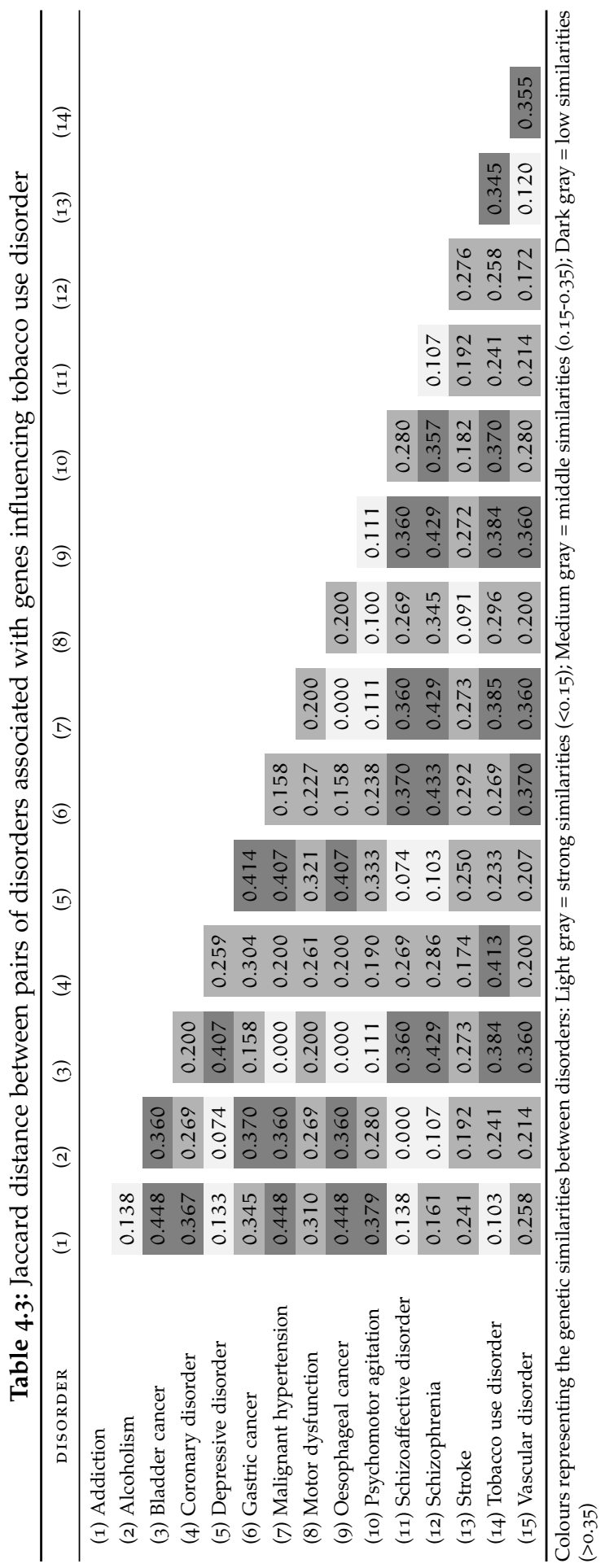


As hypothesised, the selected genes mainly influenced either the cascade theory of reward or the nicotine metabolism pathways. Most selected genes were nicotinic receptors (CHAT, CHRNA1, CHRNA2, CHRNA3, CHRNA4, CHRNA5, CHRNA6, CHRNA7, CHRNA9, CHRNA10, CHRNB1, CHRNB2, CHRNB3, CHRNB4, CHRND, $C H R N E$, and $C H R N G$ ). These receptors are activated by nicotine and a prolonged contact to nicotine will lead to receptor desensitisation [234]. Moreover, except $C H A T$, all nicotinic receptors influenced all selected disorders. There were only two genes that belong to none of these groups (SIGMAR1 and NRXN1). SIGMAR1 is a protein receptor involved in the modulation glutamatergic and dopaminergic neurotransmission [235]. NRXN1 is a presynaptic neuronal adhesion molecule. However, its biological association with multiple disorders (e.g. autism and schizophrenia) is still unclear [236].

The reason why the networks developed in IPA and STRING (Figure 4.2) are different might be due to the selection criteria. Indeed, the options in IPA are very stringent, whereas STRING used a confidence level of 0.90. Besides that, differences in the text mining algorithms used and the databases accessed by IPA and STRING may also influence the outcome.

All expected categories of disorders associated with TUD were represented in our disease selection. Due to the limitation in the number of selected disorders, to make the analyses manageable, some specific disorders associated to TUD were not selected. This is, for example, the case for lung cancer [231], attention deficit disorder with hyperactivity (ADHD) [237], and bipolar disorder [238]. McEachin et al., in their multi-gene network analysis, already reported an over-representation of genes associated to bipolar disorder and TUD. Their gene selection, developed in Gene2Mesh, included COMT, SLC6A3, and SLC6A4 [238]. Those three genes also appeared in the gene selection of the present study.

Moreover, in the literature, not only links between (i) TUD and mental illness, (ii) TUD and cardiovascular disease, or (iii) TUD and cancer are observed. For example, depression has been reported to increase the risk of cardiovascular disease. The reason of this association may be the non-compliance to treatment, shared risk factors in the two disorders (e.g. smoking and hypertension), or physiologic factors (e.g. the activation of the hypothalamic-pituitary-adrenocortical axis and inflammation) [239].

Psychiatric disorders are influenced by multiple factors and it is reasonable to think that genes do not influence psychopathology but intermediate phenotypes, such as the inter-link between dorsolateral prefrontal cortex and hippocampus or striatum. This hypothesis is supported by the following example; COMT has been consistently associated with prefrontal regulation of dopamine, which indicates a risk of schizophrenia due to the reduced signal in the prefrontal cortex [240]. However, inconsistent results were obtained when studying the direct link between COMT and schizophrenia [240]. In TUD, intermediate phenotypes may also be used to better understand the link with other disorders. In disorders that are side effects of smoking, one plausible intermediate phenotype may be tobacco smoking. 
Reward deficiency syndrome (RDS) is probably an important intermediate phenotype of coexisting disorders of smoking. RDS is a hypodopaminergic state including 4 main types of behavioural disorders: addictive behaviour (e.g. substance abuse or obesity), impulsive behaviour (e.g. attention deficit hyperactivity disorder or Tourette syndrome), compulsive behaviour (e.g. aberrant sexual behaviour or pathological gambling), and personality disorder (e.g. conduct disorder or aggressive disorder) [241]. Among others, the following variants influencing the dopamine pathway are suggested to induce RDS: DRD2 TaqIA A1, DRD2 957T, SLC6A3 VNTR $9 \mathrm{R}$, and COMT Val ${ }^{108 / 158} \mathrm{Met}$ ValVal $[241,242]$. This enhances the need of individualised interventions in case of, for example, smoking cessation. Indeed, if a smoker with depressive disorder used cigarettes as a self-medication to enhance the release of dopamine, then, during smoking cessation the treatment of depression will need adaptation [237].

Previous publications such as Carlsten et al. already pointed out the benefit of personalised medicine in TUD [243]. Due to the strong evidence that genetic factors are involved in nicotine addiction and smoking cessation, interventions should be adapted in type of treatment, dose, and duration. For example, bupropion that modifies the activity of dopamine may have an impact on smoking relapse. This may partly explain why some individuals respond better to treatments than other one [244]. Therefore, as proposed by Walton et al., there is a need to improve the 'understanding of the molecular mechanisms that underlie tobacco addiction' [244]. This understanding of the molecular mechanisms may also include those linking TUD to other disorders.

\section{Limitations}

Regarding the search strategy, the most important limitation was the low number of publications in European populations. Among other studies, there was a relatively high number of publications focusing on Asian population or on mixed ethnicities. Based on that observation, other genes may also be associated with TUD. However, the aim of the current study was not to be exhaustive in genes and disorders selection but explorative.

The use of Gene2MeSH allowed us to screen publications on PubMed in an easier way that may reduce our chance of missing publications. Looking to other databases may have increased the number of selected genes and disorders and enhanced the number of relations between selected genes and disorders. However, the addition of gene and disease selection in IPA enhanced our review as it includes text-mining research and various databases. Nevertheless, in IPA there is no possibility to limit our search based on our inclusion and exclusion criteria: focus on populations of European ancestries, and exclude studies with specific disorders or trait in the entire population. Therefore, some associations retrieved from IPA may not be specific to our criteria. 
Networks based on literature-mining, as developed in IPA and STRING (Figure 4.2), may introduce false positivity because it does not take into account the true biological relation between the elements. Further studies might take epigenomics into account to gain insight from the dynamics of the genome-environmental interactions and comorbidities. Exposomes might be linked to health effects even if to date no direct association has been reported [245]. However, genetics, epigenomics, and exposome are just part of the whole pattern explaining the relation between TUD and other disorders. This explains the interindividual differences.

\section{Implication for practice and future research}

This exploratory study may partially explain the genetic similarities between TUD and disorders using the same genetic pathways. A better understanding of the disorders linked to the same genes as TUD may help in individualising and personalising care related to TUD. Indeed, global cares developed by taking into account all the different factors that may influence a trait or a disorder, are the best practice to improve health. The use of tobacco influences, for example, the metabolism of antidepressants treatments.

In future research, the type of analyses that we developed here might be extended to individual characteristics, effects of treatments, or severity of symptoms. Moreover, the mechanisms linking TUD to disorders might be assessed in twin or prospective studies. This may give insights into whether the relation is due to causality or pleiotropy. Finally, analysis of micro-array online database for the most significant diseases may give an idea whether the expression is similar or differs between disorders. 




\section{Part II}

ENHANCING THE COMMUNICATION OF GENOMIC RISKS ABOUT SMOKING IN THE GENERAL POPULATION 

Sylviane de Viron

Johan Van der Heyden

Elena Ambrosino

Marc Arbyn

Angela Brand

Herman Van Oyen

Published as: de Viron S, Van der Heyden J, Ambrosino E, Arbyn M, Brand A, Van Oyen H. (2012) Impact of Genetic Notification on Smoking Cessation: Systematic Review and Pooled-Analysis. PLoS ONE 7(7): e40230. DOI: 10.1371/journal.pone.0040230. 
Objectives This study aimed to evaluate the impact of genetic notification of smoking related disease risk on smoking cessation in the general population. Secondary objectives were to assess the impact of genetic notification on intention-to-quit smoking and on emotional outcomes as well as on the understanding and the recall of this notification.

Methods A systematic review of articles from inception to August 2011 without language restriction was realised using PubMed, Embase, Scopus, Web of Science, PsycINFO, and Toxnet. Other publications were identified using hand search. The pooled-analysis included only randomised trials. Comparison groups were (i) high and low genetic risk versus control, and (ii) high versus low genetic risk. For the pooled-analysis random effect models were applied and sensitivity analyses were conducted.

Results Eight papers from seven different studies met the inclusion criteria of the review. High genetic risk notification was associated with short-term increased depression and anxiety. Four randomised studies were included in the pooledanalysis, which revealed a significant impact of genetic notification on smoking cessation in comparison to controls (clinical risk notification or no intervention) in short term follow-up less than 6 months ( $R R=1.55,95 \%$ CI $1.09-2.21)$.

Conclusions In short term follow-up, genetic notification increased smoking cessation in comparison to control interventions. However, there is no evidence of long term effect (up to 12 month) on smoking cessation. Further research is needed to assess more in depth how genetic notification of smoking related disease could contribute to smoking cessation. 


\section{INTRODUCTION}

Smoking is a major public health problem worldwide and the most preventable cause of mortality and morbidity. It increases the risk of many diseases such as lung cancer, chronic obstructive pulmonary diseases and cardiovascular diseases [246], but smoking cessation higly contributes to the prevention of most of these harms. Every year, around $40 \%$ of smokers attempt to quit smoking for at least one day, but only few of them succeed: approximately $2 \%$ without any help and $20 \%$ with an adequate treatment $[105,106]$. This highlights the importance of improving evidence-based interventions for smoking cessation, which could be enhanced by genetic notification of smoking related disease risk. The goal of genetic notification is to allow smokers to adapt their behaviour regarding their personal risks [247, 248].

Common diseases are highly dependent on multiple environmental and genomic factors. Both cigarette consumption and allele frequencies vary substantially between populations. Multiple single nucleotide polymorphisms (SNPs) are needed for the assessment of each specific smoking related disease risk. Generally speaking, testing multiple SNPs for diverse smoking related disease risks will identify smokers to be at higher risk of at least one disease. Different genes seem to be of interest in cancer risk prediction, among them: GSTM1, GSTT1, CYP2D6, L-myc, NQO1, and CYP1A1 [249-253].

Risk communication and health literacy are complex issues dealing with the use, the understanding and the recalling of a notification by the patient $[247,254]$. Combination of a numeric, verbal and pictorial approach maximises the understanding of the genetic risk [255]. Different models, such as the extended parallel process model [256], try to explain how people are managing information concerning their health. They highlighted that information influences emotional and cognitive representations, which could lead to an adaptive or a maladaptive change of behaviour. Genetic notification has an important psychological and emotional impact [49]. In the case of smoking, it could influence the motivation to quit smoking or lead to fear and depression symptoms that depend on the individual, the type of notification and the way it is done. Hence, being one of the core tasks of Public Health Genomics, genetic risk communication is challenging because an individual may interpret the risk as an absolute prediction. For example, he may believe that a high genetic risk of lung cancer will absolutely lead to cancer [257]. However, in general, benefits of genetic tests are more important than risks [247].

Studies reporting the impact of genetic notification on smoking cessation have been conflicting, which could, among other reasons, suggest that it is not a strong motivator of behavioural change.

The impact of genetic notification can be either explored in a real or a hypothetical situation. In hypothetical genetic testing, the anticipated reactions of smokers are assessed in view of a hypothetical genetic risk of smoking related disease. The outcome of interest is intention-to-quit smoking, which is an important precursor of 
quit attempts that lead to smoking cessation [103]. Thus improvement in intentionto-quit (e.g. enhanced by genetic notification) could be associated with an increase smoking cessation rate.

The primary objective of this systematic review and pooled-analysis was to determine the impact of genetic notification on smoking cessation in the general population. We addressed this by the following questions:

- Is genetic notification of smoking related disease risk influencing smoking cessation success rate in comparison to clinical notification of smoking related disease risk (e.g. blood pressure and cardiovascular diseases) or no notification?

- Is high genetic risk notification of smoking related disease risk influencing smoking cessation success rate in comparison to low genetic risk notification?

The secondary objectives were to review the impact of genetic notification on intention-to-quit smoking and emotional outcome as well as to determine to which extend smokers really understand and recall their genetic notification.

\section{METHODS}

First, we conducted a systematic review. Then we carried out further quantitative assessment only on the primary outcome (smoking cessation) by a pooled analysis. For this systematic review and pooled-analysis, we followed the Quality of Reporting of Systematic reviews and meta-analyses (PRISMA) guidelines [258].

\section{Eligibility criteria}

Regarding the systematic review, we included studies incorporating smokers of any age receiving genetic notification of smoking related disease risk in prospective studies (randomised, not randomised trial or cohort studies). The only exclusion criterion was studies involving hypothetical genetic notification as intervention.

The intervention of interest was the genetic notification of smoking related disease risk. Notification based on one gene was stratified in high and low genetic risk based on dominant or recessive genetic model.

The primary outcome was smoking cessation. To assess the impact of genetic notification, we collected smoking cessation rates at each follow-up that was presented in the studies. Smoking cessation could be biochemically confirmed (salivary cotinine concentration less than $15 \mathrm{ng} / \mathrm{ml}$ ) or self-reported. The outcomes in the selected studies included prolonged (continuous abstinence during a follow-up period) and point prevalence ( 1,7 or 30 -day) cessation rates. Only one study presented a sustained smoking cessation [48]. For the pooled-analysis, we focused on point prevalence smoking cessation, as this indicator was available for each study. 
Velicer et al. demonstrated a high correlation between the different types of point prevalence (24-h, 7-day, 30-day) smoking cessation ( $\mathrm{r}$ between 0.98 and 0.99) [259].

Secondary outcomes were: (a) intention-to-quit smoking; (b) emotional outcome (e.g. anxiety, depression or fear); (c) recall and understanding of the genetic notification.

\section{Search strategy}

We searched PubMed, Embase, Scopus, Web of Science, PsycINFO, and Toxnet for studies published until August 2011 without language restriction using the following terms: smoking cessation, genetic testing, and genetic predisposition to disease. The search strategy is available in the supporting information documents (Pubmed search in Supplementary material - Chapter 5). In addition, we manually reviewed the reference list of relevant articles and reviews.

Two authors (SDV and JVDH) independently screened for title and electronic abstract identified by the search for relevance to the inclusion criteria. Articles retrieved from this examination were full text screened by the same authors. Reasons for excluding studies were noted (Figure 5.1). Data were extracted by one author (SDV) and checked by the second one (JVDH). Disagreements were resolved by discussion.

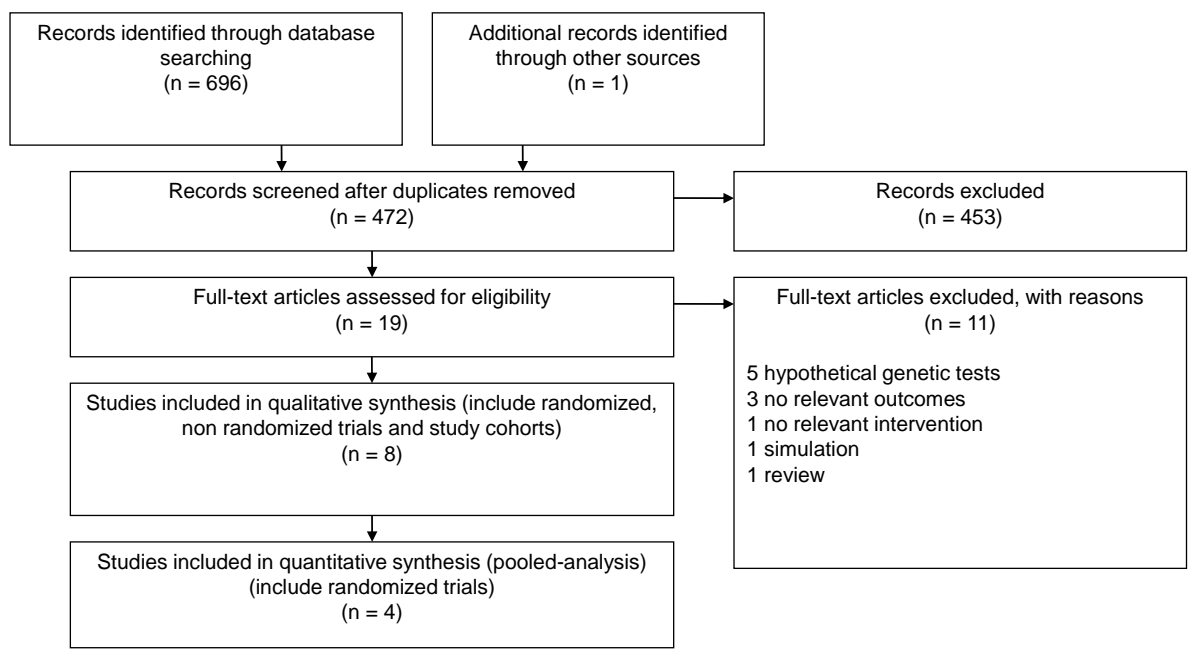

Figure 5.1: Flow chart of the study selection process 


\section{Data extraction and methodological quality assessment}

From each eligible paper of the systematic review, we extracted information, where available, on first author, year of publication, country, study setting, study design, year of the recruitment, inclusion and exclusion criteria, sample size (total and by comparison groups), description of interventions, characteristics of participants (including age, gender, ethnicity, cigarettes per day, nicotine addiction and, age of initiation), outcomes (smoking cessation, intention-to-quit smoking, emotional effects, recall and understanding of genetic testing and assessment method of the outcomes), and length of follow-up.

Methodological quality and potential risk of bias were assessed using the following criteria: selection criteria clearly described, sample size calculation, adequate allocation concealment for randomised trials, comparability of groups at baseline, presentation of the Hardy-Weinberg Equilibrium (HWE, state that allele and genotype frequency remain constant within a population); intention-to-treat analysis, ascertainment of outcome, and control for confounding.

\section{Statistical analyses}

In the systematic review, we realised comparisons of sample sizes between studies, genetic risk and gene to understand the difference in the relative proportion of smoking cessation between studies. This was effectuated using Pearson's Chisquare test. Studies included in these analyses were randomised and non randomised trial $[45,46,48,260-262]$. Sample size of high and low genetic risk was only available in four studies $[45,46,48,262]$.

Individual study risk ratios (RR) and binomial 95\% confidence intervals were computed from event numbers extracted from each study.

For the pooled-analysis, we limited included studies to randomised trials for their ability to minimise likelihood of systematic error [263]. Analyses were carried out taking into account time to follow-up: (i) Short-term follow-up was lower or equal to 6 months. (ii) Last follow-up was the last follow-up presented in the study (from 2 to 12 months). We utilised the DerSimonian and Laird method to obtain summary $\mathrm{RR}$ and 95\% confidence intervals, using random effect models for all analyses because of the important diversity between studies in the inclusion criteria or time of follow-up [264]. Statistical heterogeneity was assessed using the $\mathrm{I}^{2}$ statistic, with a value of $50 \%$ or more indicating a substantial level of heterogeneity [265]. Potential publication bias was estimated by the Egger's test. Sensitivity analysis was realised to assess the effect of each single study on the overall results by dropping one study at a time [266].

In studies that had more than two arms, we collapsed arms to obtain one intervention and one control group (e.g. collapsing no intervention and clinical risk notification group). Tests were two-sided with a significance rate $\alpha$ of 0.05 . All statis- 
tical analyses were performed using STATA, version 10.1 (STATA Corporation Inc., College Station, TX, USA).

RESULTS

\section{Description of studies}

The selection of studies included in our review is summarised in Figure 5.1. The literature search identified 696 publications from the different databases and 1 from hand search [262]. The publication retrieved from hand search was in the reference list of Hishida et al. [45]. After removal of duplicate references, 472 were included. A total of 453 were discarded in the title and abstract screening because these papers did not meet the criteria. From the 19 studies included in full text review, 11 did not meet the inclusion criteria as described [50-52, 267-274]. Finally, 8 papers from 7 studies met the inclusion criteria (Table 5.1) [43, 45-48, 260-262]. The studies of Lerman et al. [261] and Audrain et al. [260] were based on the same population but presented the outcomes at two and twelve months of follow-up, respectively. Four studies had recruited their participants in 2000 and later $[43,45-47]$ and three studies recruited before $2000[48,260,261]$. In the last study, the recruitment period was not reported [24]. Studies took place in the UK [262], the US [48, 260, 261] and Japan [43, 45-47]. Study participants were recruited via newspapers advertisements [260, 261], university [41], annual check-up of employees [43, 45, 47], outpatients consulting general practitioners or specialists $[46,48]$, smoking clinic [261] or telephone quit smoking service [262]. The sample sizes ranged from 61 to 697 and the follow-up ranged from 2 to 12 months.

Participants were aged from 18 to 88 years old. The percentage of females was around $50 \%$ except in two studies where there were only $6.2 \%$ and $14.0 \%$ females $[45,47]$. In general, participants had to smoke at least 1 cigarette per day (CPD) or 7 cigarettes per week to be recruited $[46,48,262]$. Four studies presented the mean CPD of their participants this ranged between 15.5 and $22.7[45,48,261,262]$. Two studies enrolled patients that wanted to quit smoking [260, 261], whereas, in the other studies, participants were not necessarily trying to quit smoking [43, 45, 47, 48]. 


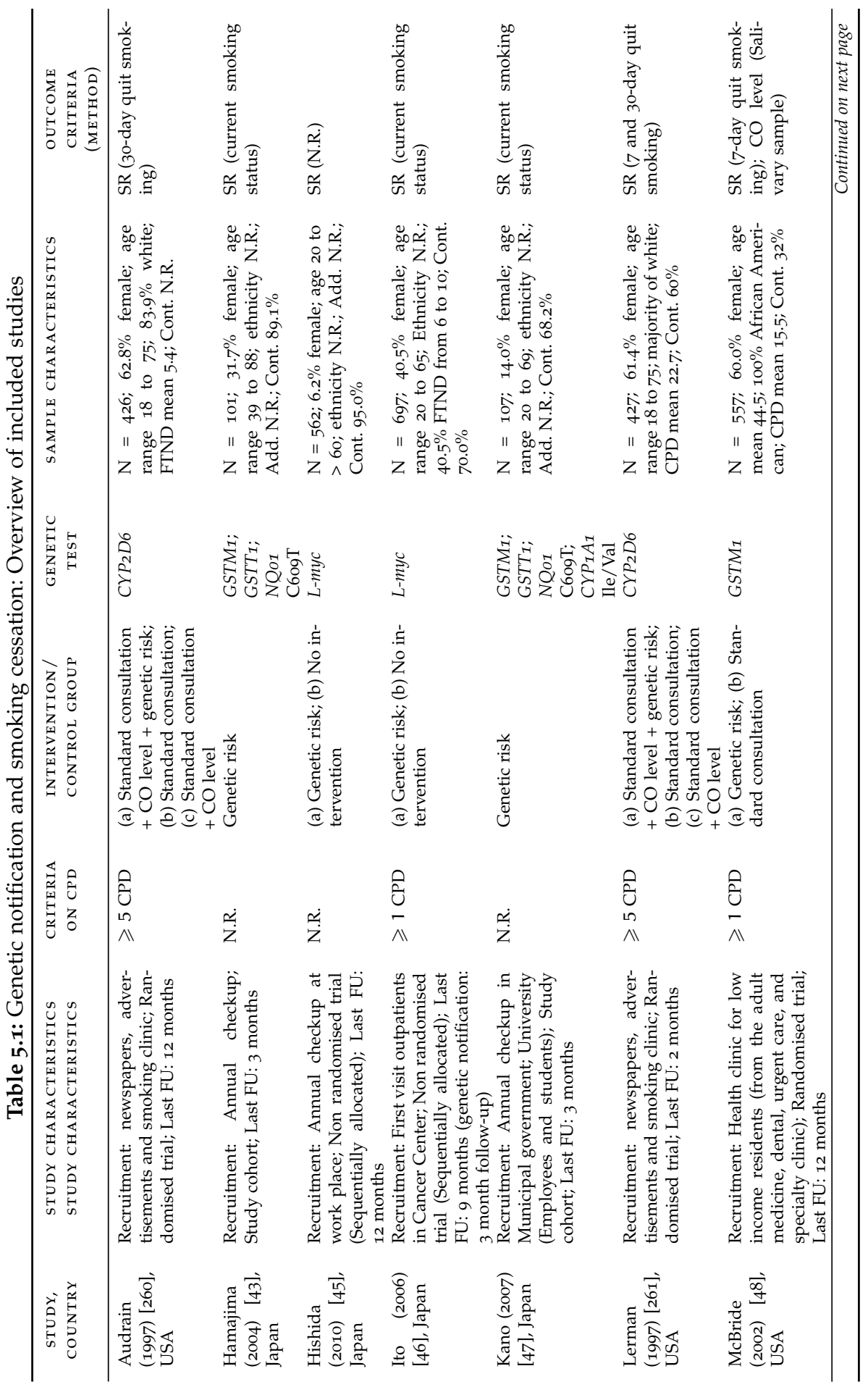


IMPACT OF GENETIC NOTIFICATION ON SMOKING CESSATION | 83

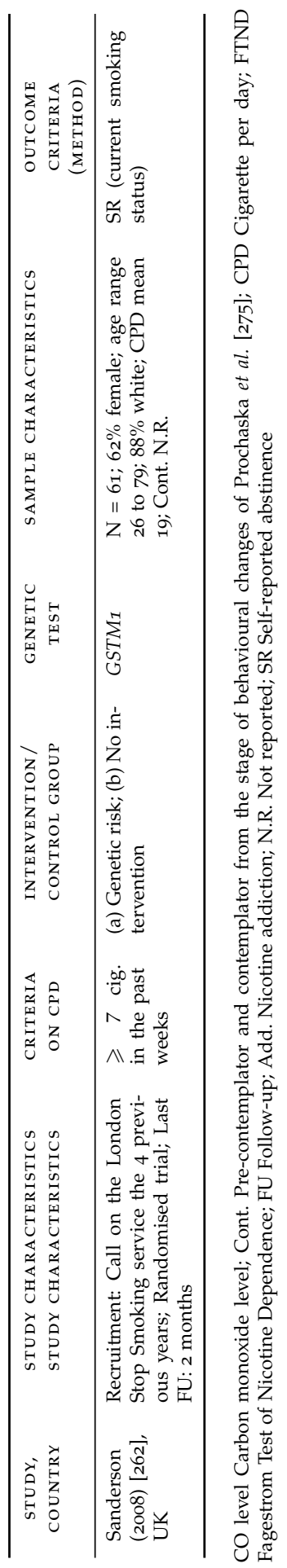


Regarding the study design, four studies were randomised trials [48, 26o-262], two studies did not randomise their interventions (sequential allocation) [45, 46], and the remaining two were cohort studies $[43,47]$. Four studies compared groups that could receive genetic notification to control $[45,46,48,262]$. The last two trials had three arms (i) standard quit smoking consultation (QSC) to (ii) clinical risk notification, which consisted of 10 minutes of motivational intervention including carbon monoxide (CO) level prior QSC, and (iii) genetic risk notification, which consisted in personalised feedback of genetic test, QSC and clinical risk notification $[260,261]$.

The disease of interest was always cancer. In six studies risk notification was based on a single-gene test (GSTM1, L-myc or CYP2D6) [45, 46, 48, 260-262]. In one study three genetic tests (GSTM1, GSTT1 and NQo1 C609T) [43] were involved. In the study of Kano et al. CYP1A1 Ile/Val was added to the previous list [47].

\section{Quality of studies and publication bias}

A summary of the risks of bias for the included studies in Table 5.3 (Supplementary material - Chapter 5 ). One study reported allocation randomisation procedures in sufficient details (including the explanation of the procedure for the randomisation) [262], whereby intervention allocation were not known or predicted by the participants or the medical support teams before their assignments. The 3 other randomised studies did not mention the procedure of randomisation [48, 260, 261]. Studies in which interventions were randomised according to week or month of attendance $[45,46]$, were considered as non randomised studies in the analyses. Half of the studies reported the sample size calculation [46, 260-262] and one clearly reported the test of HWE [45]. Smoking cessation was sometimes defined as a continuous abstinence [46], 7-day abstinence [48, 261], 30-day abstinence [46, 260, 261], or 'current smoking status' [43, 45-47, 262]. Only one study tried to confirm biochemically the self-reported abstinence but the return rate of the samples was only $39 \%$. Thus they decided not to use this outcome and to use only self-reported cessation [48]. Six studies controlled their results for confounding [45, 46, 48, 260-262].

Egger's two tailed p-value showed no significant publication biases for short follow-up as well as the last follow-up of main analyses (genetic notification versus control) (respectively, $P=0.11$ and $P=0.76$ ).

\section{Primary outcome: Smoking cessation}

The impact of genetic notification on smoking cessation was conflicting among randomised and non randomised studies [45, 46, 48, 260-262] although the results were not significant. Three studies displayed a higher smoking cessation rate in the genetic notification group than in the control group $[48,261,262]$ and three other studies reported the opposite $[45,46,260]$ (Table 5.2). Figure 5.2 indicated that, the distribution of high and low genetic risk was significantly different between genes 
(L-myc and GSTM1; $P<0.001)$ and between the four different studies $(P<0.001)$ but not between authors within genes (GSTM1 $P=0.10$; L-myc $P=0.79$ ). For GSTM1, more participants had a lower genetic risk and for $L-m y c$, more participants had a higher genetic risk. However, distribution of high and low genetic risk notification were not available for two studies [260, 261].

Table 5.2: Risk ratios and $95 \%$ confidence intervals associated with smoking cessation following intervention (genetic notification versus control)

\begin{tabular}{rcc}
\hline \multicolumn{1}{c}{ AUthor } & GENE & RISK RATIo (CI 95\%) \\
\hline Audrain (1997) & CYP2A6 & $0.73(0.41-1.28)$ \\
Hishida (2010) & L-myc & $0.75(0.40-1.41)$ \\
Ito (2006) & L-myc & $0.90(0.66-1.24)$ \\
McBride (2002) & GSTM1 & $1.47(0.90-2.39)$ \\
Lerman (1997) & CYP2D6 & $1.44(0.74-2.80)$ \\
Sanderson (2008) & GSTM1 & $0.92(0.37-2.27)$
\end{tabular}

Risk ratios higher than one mean a positive effect of genetic notification on smoking cessation.

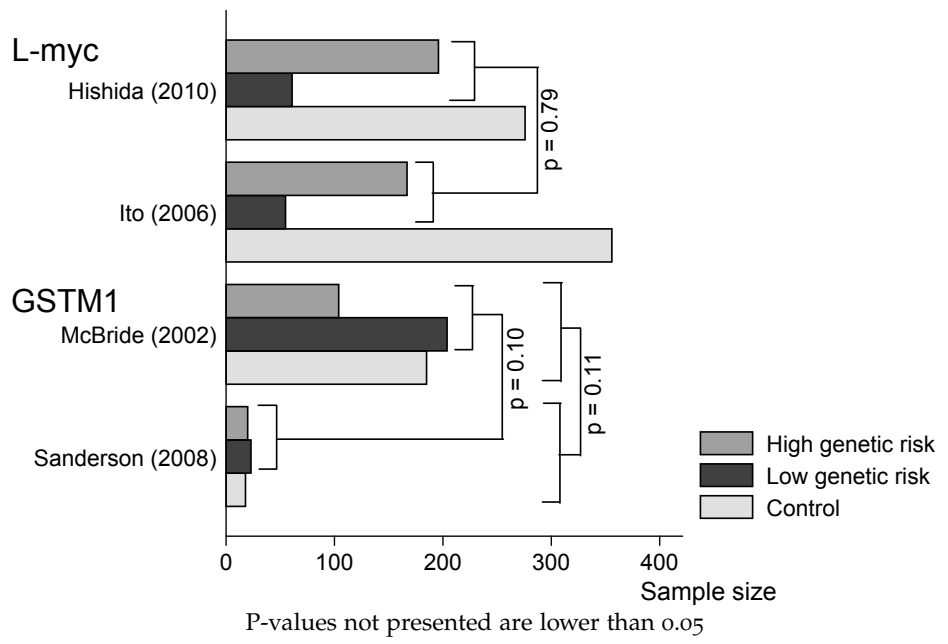

Figure 5.2: Comparison of the distributions (High, low genetic risk and/or control) between studies or genes

The pooled analysis indicated that, when considering the last follow-up, there were no significant differences in smoking cessation between the following subgroups: genetic notification versus control, low genetic risk notification versus control, and high genetic risk notification versus low-genetic risk (respectively, RR (95\% CI) $1.03(0.64-1.65)$; 0.97(0.33 - 2.88); 1.48(0.74-2.95)) (Figure 5.3). However, compared to the control group high genetic risk notification was borderline associated with an increased smoking cessation $(R R=1.62(0.98-2.67))$. No heterogeneity was observed across studies in the different pooled-analyses ( $\mathrm{I}^{2}$ range from 0.0 to $42.9 \%$, 
$P$ range from 0.17 to 0.54 ) except a substantial heterogeneity in the low genetic risk notification versus control group $\left(\mathrm{I}^{2}=61.4 \%, P=0.11\right)$.

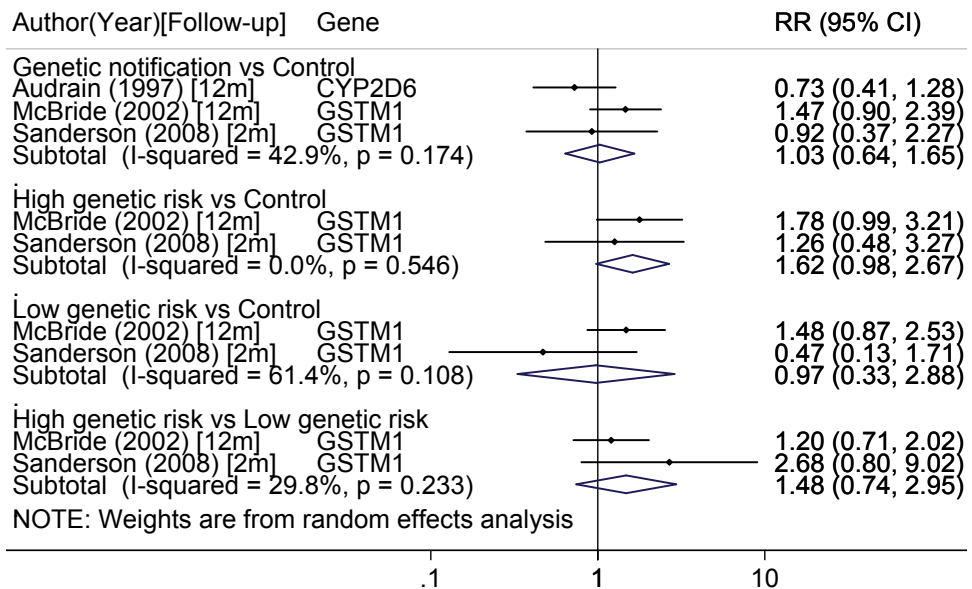

Genetic notification group versus control; High genetic risk versus control; Low genetic risk versus control; High genetic risk versus low genetic risk. Risk ratios higher than one mean a positive effect of genetic notification on smoking cessation

Figure 5.3: Pooled-analysis of smoking cessation associated with genetic notification in randomised trials for the last follow-up (2, 6 or 12 month)

When focusing only on short-term smoking cessation outcome (2 or 6 month), the borderline effect of high genetic risk versus control was still visible $(\mathrm{RR}=$ $1.55(0.94-2.58))$. Moreover, genetic notification increased 1.55 times smoking cessation in comparison to control $(\mathrm{RR}=1.55(1.09-2.21)$ (Figure 5.4). These two analyses seemed fairly homogeneous $\left(\mathrm{I}^{2}=0.0 \%\right.$ for the two analyses). Sensitivity analyses did not identify influential studies.

\section{Secondary outcomes}

Secondary outcomes were available for most of the studies but not all of them.

(a) Intention-to-quit. Six studies observed intention-to-quit smoking after a genetic notification of smoking related disease risk [43, 45-48, 262]. However, different notions were used: 'wish to quit' [43, 45, 47], 'desire to quit' [48] and 'motivation to quit' $[46,262]$. Most studies just evaluated intention-to-quit smoking at baseline. Four studies presented multiple evaluations over time, such as at baseline, before genetic announcement and three months after announcement; or at baseline, and one week and two months after $[43,45,47,262]$. Two studies indicated no difference in intention-to-quit at different time of follow-up [45, 262]. Although, one reported a significant difference in motivation to quit smoking at one week $(P=0.003)$ but no more at two months [262]. Nevertheless, Ito et al. reported no difference of motivation to quit smoking between high and low genetic risk of smoking related disease 


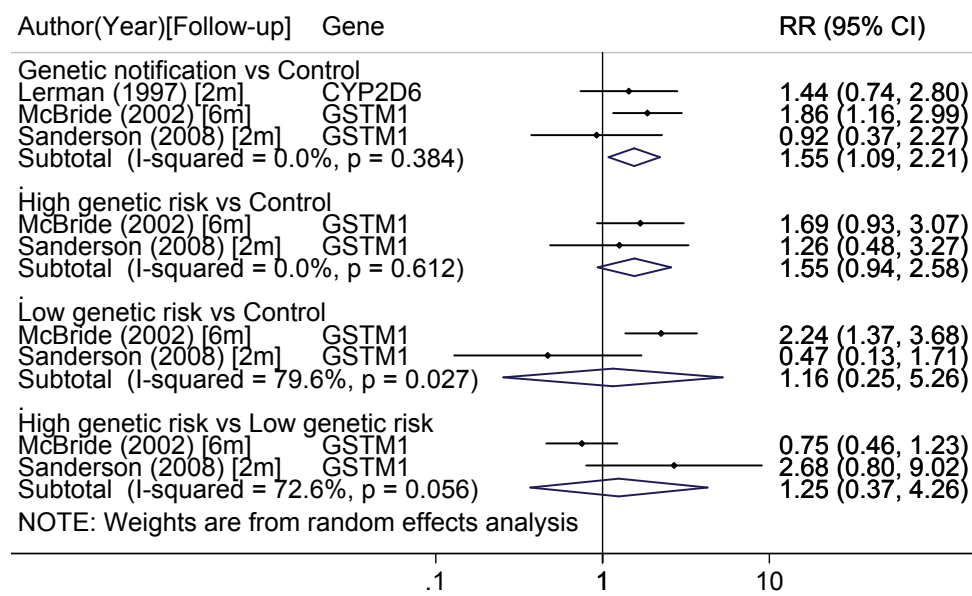

Genetic notification group versus control; High genetic risk versus control; Low genetic risk versus control; High genetic risk versus low genetic risk. Risk ratios higher than one mean a positive effect of genetic notification on smoking cessation

Figure 5.4: Pooled-analysis of smoking cessation associated with genetic notification in randomised trials for follow-up $\leqslant 6$ month ( 2 or 6 month)

$(P=0.18)[46]$. In the 6 studies assessing the stage of change, the majority of the population across 5 studies was pre-contemplator or contemplator (no concern or no intention-to-quit smoking): $89.1 \%$ [43], 95.0\% [45], 68.2\% [47], 60\% [261], and 70\% [46]. In only one study this percentage was low (32\% [48]) (Table 5.1).

(b) Emotional outcome. Seven studies reported emotional outcome [43, 45-48, 261, 262]. Participants receiving genetic notification were more likely to report shortterm depression, anxiety or fear arousal than others $[45,261,262]$. More specifically participants with high genetic risk reported increased fear arousal than those with low genetic risk $(P<0.01)$ [46]. This result was not observed in the study of McBride et al. [48] and was not significant anymore at 2 months follow-up in the study of Sanderson et al. [262]. Anxiety was not significantly different across comparison groups [262].

(c) Recall and understanding. Smokers seemed to reasonably understand the meaning of their genetic test results [262]. McBride et al. reported that half of their participants read the biomarker test result booklet and that an equal number of participants from the high genetic risk and low genetic risk groups interpreted accurately their results (respectively, 56 and 53\%) [48].

\section{DISCUSSION}

Our systematic review indicated that few studies have assessed the impact of genetic notification on smoking cessation. Only 8 papers based on 7 different studies were available and just 4 of them were included in the pooled-analysis. 
The pooled-analysis suggested a short-term increase of smoking cessation for participants receiving genetic notification in comparison to control group and a borderline increase of smoking cessation for high genetic risk smokers in comparison to control. No evidence of heterogeneity was observed for these results even if (i) characteristics of participants, (ii) inclusion criteria and (iii) study designs differed among studies. For example, (i) included participants were not necessarily interested in smoking cessation. Furthermore, participants were not necessarily from the same ethnicity, although mixing ethnicities in genetic studies could lead to population stratification. The majority of studies did not report HWE calculation. (ii) Inclusion criteria differed in number of CPD and none of the studies met the criterion of nicotine addiction, often defined as smoking more than 10 CPD for at least 12 months $[71,76,80]$. (iii) Control groups in the study design received either no intervention $[48,262]$ or clinical risk notification and no intervention [260, 261]. These factors may have affected our results. However, for example for the control groups, collapsing no intervention to clinical risk notification did not introduce a significant change in comparison to no interventions alone for both Audrain et al. [26o] or Lerman et al. [261]. Moreover, this also did not influence significantly the results of the total pooled analysis.

The reason why some results of the pooled-analysis were significant and others were not remains open to interpretation. The impact of genetic notification is likely to vary with individual characteristics (e.g. willingness to quit smoking, socioeconomic status or health literacy), with the distribution of high and low genetic risk notification, with the way in which the genetic notification is done (e.g. counseling before genetic notification, only oral explanation or leaflet with graphical illustration), the intervention in the control group, and the length of the follow-up. Due to the low number of included studies, it was not possible to stratify the pooledanalyses by time of follow-up ( 2,6 or 12 months). Stratification of the results before and after 6 months follow-up should be also of interest. However, after 6 months there were only 2 studies available for genetic notification versus control [48, 26o] and only one for the stratified analyses (high genetic risk versus control; low genetic risk versus control; high versus low genetic risk) [48]. This enhances the need of increasing the follow-up of studies about the impact of genetic notification on smoking cessation. The inclusion of new studies in the pooled-analysis could improve the power of the analyses. This could either confirm our current results or in the opposite, present a significant impact of genetic notification in long-term follow-up. However, current results do not demonstrate any evidence of long term effect of genetic notification on smoking cessation. And in a population receiving high genetic risk notification, only one study reported a marginally non-significant long-term effect [48].

In a recent meta-analysis the same primary outcome was studied, but there were differences in the method that was used [155]. In our study, we decided to focus on randomised trials only for their ability to minimise the likelihood of systematic errors. Smerecnik et al. included both randomised [48, 260-262] and non randomised 
trials $[45,46]$. In order to avoid overestimation of a single study we considered one follow-up of each study in the pooled analysis (last follow-up or short-term smoking cessation), whereas this was not the case in the other study. We decided to use RR rather than OR for their easiness of interpretation and for their improved accuracy in prospective studies. In our study we also assessed secondary outcomes (intention-to-quit smoking, emotional outcome and recall and understanding of the genetic information). Despite the differences the main outcome of both studies is similar, which reinforces the validity of the results.

Genetic notification did not influence intention-to-quit smoking, except in one study reporting motivation to quit at one week follow-up [262]. In hypothetical genetic tests, higher anticipated intention-to-quit was reported in genetic notification in comparison to the control group [50] and in the high genetic risk group in comparison to the low genetic risk group [51, 267-269]. This discrepancy seemed to demonstrate that the anticipated reaction in hypothetical genetic tests did not represent reality. However, this could be due to divergence in the presentation and the understanding of genetic notification or differences in the characteristics of the population. The emotional outcome could also influence this result. In hypothetical genetic tests, smokers will probably be less influenced by depression, anxieties and fear arousal than in real genetic tests. Thus, how smokers recall and understand genetic notification as well as how they are influenced by emotional outcome could improve the use of this intervention in smoking cessation. In the pooled-analysis, most participants were in quite strong intention-to-quit at baseline [48, 262]. However, this variable was not assessed in the 2 last studies [260, 261].

Most studies were testing only one single gene to determine smoking related disease risk. This posed also ethical questions because of the uncertainty of disease risk, which is enhanced when using only single gene test in common diseases.

Genetic notification is one possible intervention among others. At the individual level, the most well-known one are the pharmaceutical interventions (nicotine replacement therapies, Bupropion or Varenicline). However, nicotine dependence is not only a physical dependence but also a behavioural and a psychological dependence. Consequently, interventions might take into account these three types of dependence (e.g. multidisciplinary follow-up including psychological counseling and pharmaceutical treatments). Other interventions are also available at the household level (e.g. smoke-free home and partner support) and the society level (e.g. mass media, package warning, and bans). As the evidence for benefit of these interventions is strong and well-established, it is incumbent upon genetics to demonstrate additional benefit [243].

\section{Limitations of the review}

Regarding the pooled-analysis, the most important limitation was the low number of included studies, which did not allow us to determine whether the risk varied with particular conditions (e.g. history of smoking related disease or stage of 
behavioural change of Prochaska et al. [275]). This low number of included publications was even more present in the pooled-analysis. This is also explained by the fact that we would include only randomised trials that are known to be of higher quality [263]. Publication biases are in general the principal methodological limitation in meta-analyses. It is possible that we missed unpublished reports. The Egger statistical analysis, which is a test for publication biases, suggested that there were no small study effects ( $p$-value comprised between 0.11 and 0.76 ). However, the sensitivity of this test is generally low in meta-analyses based on fewer than 20 studies [276]. Finally, we did not control our results for multiple testing.

Some limitations pertained to the studies themselves. The outcome measures differed across the studies (e.g. smoking cessation: prolonged abstinence or different point prevalence abstinence). Moreover, adjusted RRs were rarely presented in the included studies, which prevented control for confounding factors. Another limitation was that interventions in the control groups were not similar in the different studies included in the pooled-analysis: two studies had a control group without any intervention $[48,262]$ and the two others had two control groups (no intervention and clinical risk notification) [260, 261]. The latter have been collapsed in the pooled-analyses, which might dilute the effect of genetic notification on smoking cessation.

Finally, limitations are also due to the heterogeneity between the included studies. This is due to the diversity in the inclusion criteria. For example, the mean number of CPD ranged from 15.5 [48] to $22.7[260,261]$ depending on the study. Nevertheless, to take this problem into consideration we used random effect models in the pooled-analyses.

\section{Implications for practice and research}

The results from this study suggest that genetic notification of smoking related disease risk could have a positive impact on smoking cessation, particularly in short-term follow-up. To determine the possible implications for practice, further research of the impact of genetic notification on smoking cessation is needed. There is also need to investigate the cost-effectiveness of this intervention. Studies should (i) focus on smokers that want to quit smoking, (ii) focus on population of regular smokers by level of severity of nicotine addiction, (iii) use combination of genetic tests for a single or multiple smoking related diseases, (iv) standardise different concepts (e. g. smoker, addiction, intention-to-quit, and smoking cessation) to minimise the heterogeneity and risk of bias between studies. 
SUPPLEMENTARY MATERIAL - CHAPTER 5

PubMed search

The research on Pubmed was realised using the following code:

((smoking cessation) AND ((genetic testing) OR genetic predisposition to disease)))

Quality assessment of studies included in the systematic review of genetic notification

Table 5.3: Quality assessment of studies included in the systematic review of genetic notification

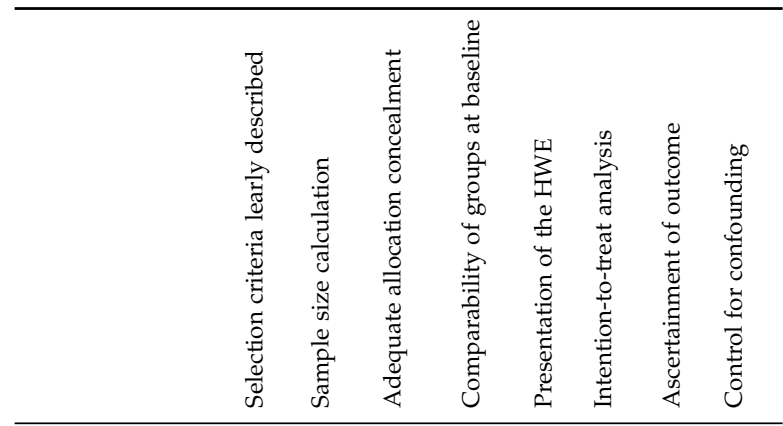

\begin{tabular}{|c|c|c|c|c|c|c|c|c|}
\hline \multicolumn{9}{|c|}{ Studies included in the systematic review and pooled analysis } \\
\hline Audrain 1997 & + & + & $\pm^{1}$ & + & - & - & -3 & + \\
\hline Lerman 1997 & + & + & $\pm^{1}$ & + & - & - & -3 & + \\
\hline McBride 2002 & + & - & $\pm^{1}$ & $\pm^{2}$ & - & + & + & + \\
\hline Sanderson 2008 & + & + & + & + & - & + & -3 & + \\
\hline \multicolumn{9}{|c|}{ Studies just included in the systematic review } \\
\hline Hamajima 2004 & - & - & $\varnothing$ & $\varnothing$ & ? & - & -3 & - \\
\hline Hishida 2010 & - & - & - & + & + & - & -3 & + \\
\hline Ito 2006 & \pm & + & - & + & - & + & -3 & + \\
\hline Kano 2007 & - & - & $\varnothing$ & $\varnothing$ & - & + & -3 & - \\
\hline
\end{tabular}

+ Yes; - No or not reported; ? unclear; ø Not applicable; ${ }^{1}$ No explanation on how the randomisation was processed; ${ }^{2}$ Significant difference in desire to quit at baseline (but not corrected for multiple testing); ${ }^{3}$ Not even try to confirm the smoking status biochemically 

COMMUNICATING GENETICS AND SMOKING THROUGH SOCIAL MEDIA: ARE WE THERE YET?

Sylviane de Viron L. Suzanne Suggs Herman Van Oyen Angela Brand

de Viron S, Suggs LS, Brand A, Van Oyen H (2013) Communicating genetics and smoking through social media: Are we there yet? J. Med. Internet Res. 15(9):e198. DOI: 10.2196/jmir.2653. 
Background Social media is a recent source of health information, which could disseminate new scientific research, such as genetics of smoking.

Objectives Therefore, the objectives were (1) to evaluate the availability of genetic information about smoking on different social media (YouTube, Facebook and Twitter) and (2) to assess the type and the content of the information displayed on the social media as well as the profile of people publishing this information.

Methods We screened the posts on YouTube, Facebook and Twitter with the following terms 'smoking' and 'genetic' in two time points (September 18, 2012 and May 7 , 2013). The first 100 posts were reviewed for each media for the time points. Google was searched during Time 2 as an indicator of available information on the web and the other social media discussing genetics and smoking. The source of information, the country of the publisher, characteristics of the posts, and content of the posts were extracted.

Results In YouTube, Facebook and Twitter, respectively, 31, o and 84 posts were included. Posts were mostly based on addiction smoking related diseases, referring to scientific publications and the publisher was mostly from the US. In Google search, most results were scientific databases. Six scientific publications referred to within the Google search were also retrieved on either YouTube or Twitter.

Conclusions Despite the importance of public understanding of smoking and genetics and the high use of social media, little information is present on social media. Therefore, there is a need to monitor the information and to evaluate the understanding of the population about information related to genetics and smoking displayed on social media. 


\section{INTRODUCTION}

Social media are increasingly recognised as important tools for information provision, gathering and transfer. Social media allow the spread of information to many people through different means [277]. For example, someone can publish a video on YouTube where anyone can view, listen, and even download the video. They can also create a group on Facebook to promote that video. In social media, every individual, regardless of credentials, is able to post and retrieve such information. Therefore, available information on social media is not exclusively based on expert's knowledge but both on experts' and laypersons' experiences [278].

Health consumers use social media for a variety of purposes. A recent consumer survey observed that $24 \%$ of consumers posted information about their health experiences on social media platforms. Sixteen percent of consumers posted reviews of medications, treatments, doctors or health insurers. Health symptoms or behaviour were traced and shared for $18 \%$ of the consumers. Health-related causes were joined by $20 \%$ of the consumers and supported by $28 \%$. Further, $16 \%$ of consumers share health-related videos or images on social media. Consumer trust in information posted on social media varied by messenger source ranging from hospitals $(55 \%)$, from others they know $(46 \%)$, health insurance companies $(42 \%)$, and from unknown patients $(25 \%)$. Regarding their susceptibility to share their own health information on social media, 30\% would share this information with other patients, $43 \%$ with hospitals, and $38 \%$ with health insurance companies [278, 279].

To date, most studies assessing the exposure to information about smoking on social media focused on pro- and anti-smoking information. Among adolescents, exposure to tobacco content appeared to be limited in volume with $43 \%$ of adolescents being exposed to a mean of 13 pages of pro-tobacco content during 1-month follow-up [28o]. The rate was nearly similar for anti-tobacco content, with $45 \%$ of the adolescents exposed to a mean of 10 pages of anti-tobacco content [280]. Some studies focused on the content of posts on YouTube specifically, most of them being on tobacco control. But other topics where also developed: anti-smoking and quit smoking posts as well as smoking sexual fetish posts [278]. Facebook and Twitter are important ways to monitor the tobacco industry and to facilitate tobacco control [281]. As proposed by Hefler et al. social media strategies may be integrated into tobacco control organisations [281]. Moreover, social media, such as Facebook, already include information about many disorders and genetic syndromes [282].

Given the use of social media for health purposes and the increasing research, academic papers, and public and policy attention to genetic testing and genetic relationships with disease, it is expected that social media are likely to become an important source for obtaining and disseminating genome-based information [33].

Despite the vast amount of research and efforts to prevent smoking and support cessation, smoking is still a major public health problem worldwide. Factors influencing smoking behaviour are both non-genetic and genetic. Non-genetic factors included a broad range of aspects, such as social factors (e.g. smoking status of 
peers), economic factors (e.g. level of income), or psychological factors (e.g. weight concerns). For genetic factors, the two main factors are the genes influencing the nicotine metabolism and genes influencing the cascade theory of reward [221].

Genetics and smoking are highly covered topics on different media. For example, a search for 'genetics' and 'smoking' on PubMed (including scientifically based content) resulted in 15,948 results. A search on Google (including both scientifically and non-scientifically based content) revealed 9,970,00o hits. On YouTube, 1,300,000 posts were obtained, on Facebook 472,000, and 8,020 on Twitter (using the searches, 'smoking + genetic + site: YouTube.com', 'smoking + genetic + site: facebook.com', and 'smoking + genetic + site: twitter.com'). We also conducted a search of a specialised social media platform, PatientsLikeMe, and found 167 hits. However, upon review of posts, only one publication about chronic obstructive pulmonary disease (COPD) and genetics was listed (and this publication was displayed multiple times). The high number of results in both scientific and non-scientific search sources suggests that such information could also be available in popular social media and could be found by lay public using typical simple search strategies.

Given the reach of social media and the growing reliance on it for health purposes, combined with the importance of genetics and smoking, this study aims to explore the availability of genome-based information about smoking on three popular social media platforms (YouTube, Facebook and Twitter). Questions examined include,

1. What type of information about genetics and smoking is displayed on social media?,

2. What is the source (scientific or non-scientific) of the posted information?,

3. What is the role of the publisher?, and

4. What countries is information being posted from?

We expected that the information would be posted primarily from scientific sources from research centers in the United States and Europe.

\section{METHODS}

\section{Sample}

Posts from YouTube, Facebook and Twitter were included. YouTube (video sharing), Facebook (social networking) and Twitter (social networking and microblogging) are each ranked among the top 10 most popular websites [283], Facebook was positioned second place just after Google. YouTube and Twitter were respectively at positions 3 and 8 . The other websites included in the top 1o were retail websites (e.g. Amazon . com) or Web-search engine (e.g. Google). Therefore, YouTube, Facebook and Twitter appeared to be the most relevant social media. The number of users on social media is growing daily, however Facebook is still the top used medium with 
around 1.01 billion active users in 2012 [284]. Twitter counts roughly 500 million users and 200 million active users [285], and over 800 million unique users visit YouTube [286] each month.

\section{Search strategy}

The terms 'genetic' and 'smoking' were searched for on each of the three social media. These terms were selected because they were simple terms that the general population may use to get information on the topic. The search was performed using two time points, the first one on September 18, 2012, and the second on May 7,2013 . The first 100 posts available for each social media platformwere examined. Posts on YouTube were searched with the 'relevance' option (the default option). On Facebook, posts were searched using both the total results and results by 'people', 'pages', 'groups', 'apps', 'events', 'music', 'public posts', and 'posts in groups'. On Twitter, only the posts of the previous days (approximately one week depending on the storage capacity of Twitter's database) were visible at Time 1 . This changed at Time 2, when posts from the previous months were available. Posts were excluded if they did not express the link between genetics and smoking tobacco (including smoking initiation, addiction, cessation and smoking related diseases). Figure 6.1 shows an example of the posts retrieved on YouTube and Twitter.

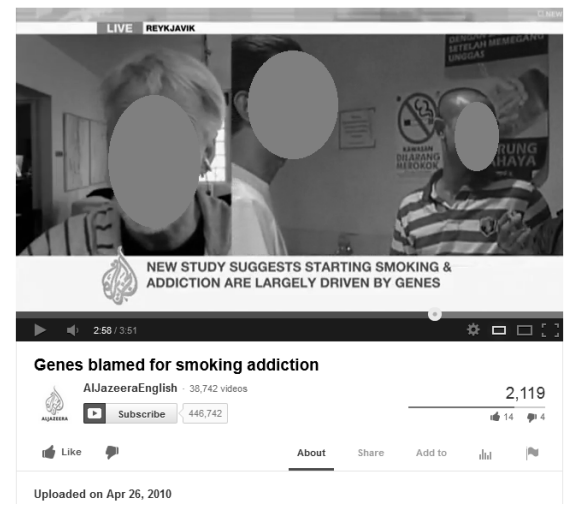

(a)

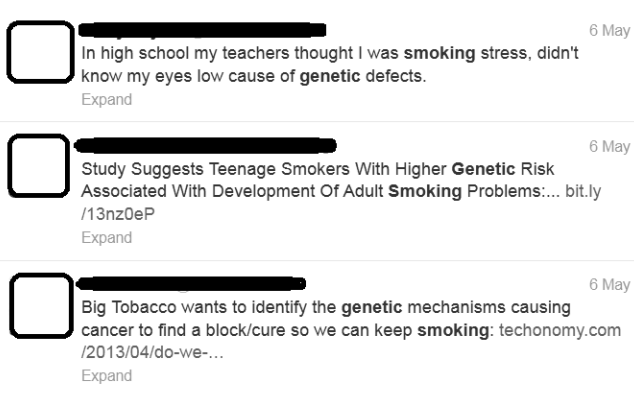

(b)

(a) is the screenshot of YouTube (b) is the screenshot of Twitter.

Figure 6.1: Screenshots of YouTube and Twitter results

Moreover, the first 100 posts were searched on Google at Time 2 using the same search terms. The aim of searching posts on Google was twofold, firstly to indicate the type of information available on the Internet and secondly to determine if there were other social media that discussed genetics and smoking (e.g. health forums or blogs). 


\section{Data extraction}

From the different posts, the date of the publication and the country of the publisher were extracted. To understand, at least partially, the credibility of the information provider, the role of the publisher was coded and classified as a research center, news, medical news, independent user (i.e. meaning that the person posting on the social media was acting as an individual citisen and not on behalf of a group of people or organisation or as a scientist) and other, if it belonged to none of the previous categories (e.g. foundation such as 'Arthritis Foundations' or companies such as '2zandme'). The source of posted information was categorised as 'scientific publication', 'referring to a scientific publication' and 'non-referring to a scientific publication'. This allowed us to understand the differences in types of content posted on the three social media. The content of the posts was classified into smoking initiation, addiction, cessation, and smoking related diseases. At least a link between genetics and the specific category must have been mentioned to allow the classification. Moreover, one post may have been classified in more than one category. For smoking related diseases, we extracted the disease of interest. When available, we extracted the number of views and the opinion (like or dislike) of the post.

\section{Statistical analyses}

Univariate analyses were assessed by the Pearson's Chi square for categorical data and the Kruskal-Wallis one-way analysis of variance for continuous data (because the continuous variables were not normally distributed). Tests were two-sided with a significance rate $\alpha$ of 0.05 . Tests were corrected for multiple testing through Bonferroni-Sidak. P-values lower than o.001 remained significant after correction for multiple testing. All statistical analyses were performed using STATA, version 10.1 (STATA Corporation Inc., College Station, TX, USA).

To show the content of the posts on Twitter, the titles of the posts on YouTube, and the most frequent words found in the titles of the Google search, word clouds were used. Word clouds visually represent the frequency of the words used in the posts with larger size for more frequent words. Word clouds were created with the 'wordcloud' package using the $\mathrm{R}$ project for statistical computing ( $\mathrm{R}$ version 2.14.1; http://www.R-project.org). 
Characteristics of the three social media

Across both data collection points, YouTube, Facebook and Twitter retrieved, respectively, a total of 200, 0 and 171 posts among them 31, o and 84 discussed genetics of smoking. On YouTube 16 posts were retrieved both at Time 1 and 2. Moreover, from the 9 posts selected at Time 2, three were published after Time 1. By contrast, Time 1 (September 2012) and 2 (January to May 2013) did not overlap on Twitter. Therefore, no posts were found at both data collection points on Twitter (Figure 6.2). The number of included and excluded posts was significantly different between the three different social media $(P<0.001)$. Twitter obtained a higher proportion of posts discussing genetics and smoking (49.1\% in comparison to $15.5 \%$ on YouTube).

a.

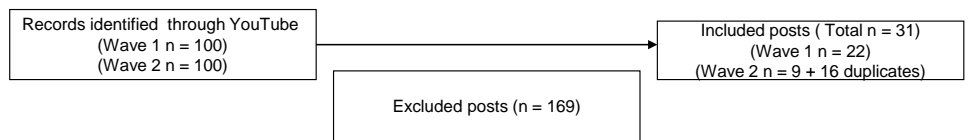

b. Records identified through Facebook

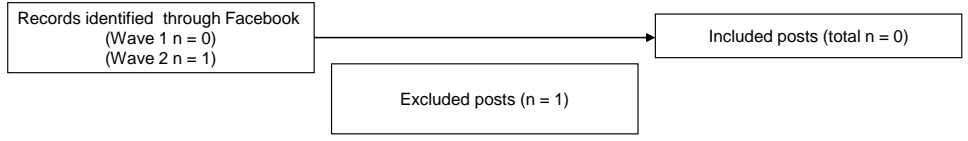

c.

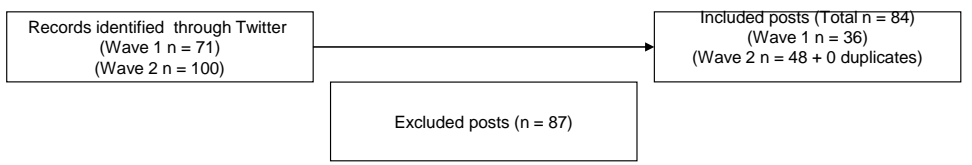

(a) is the YouTube flow chart; (b) is the Facebook flow chart; (c) is the Twitter flow chart.

Figure 6.2: Flow Chart of the post selection 


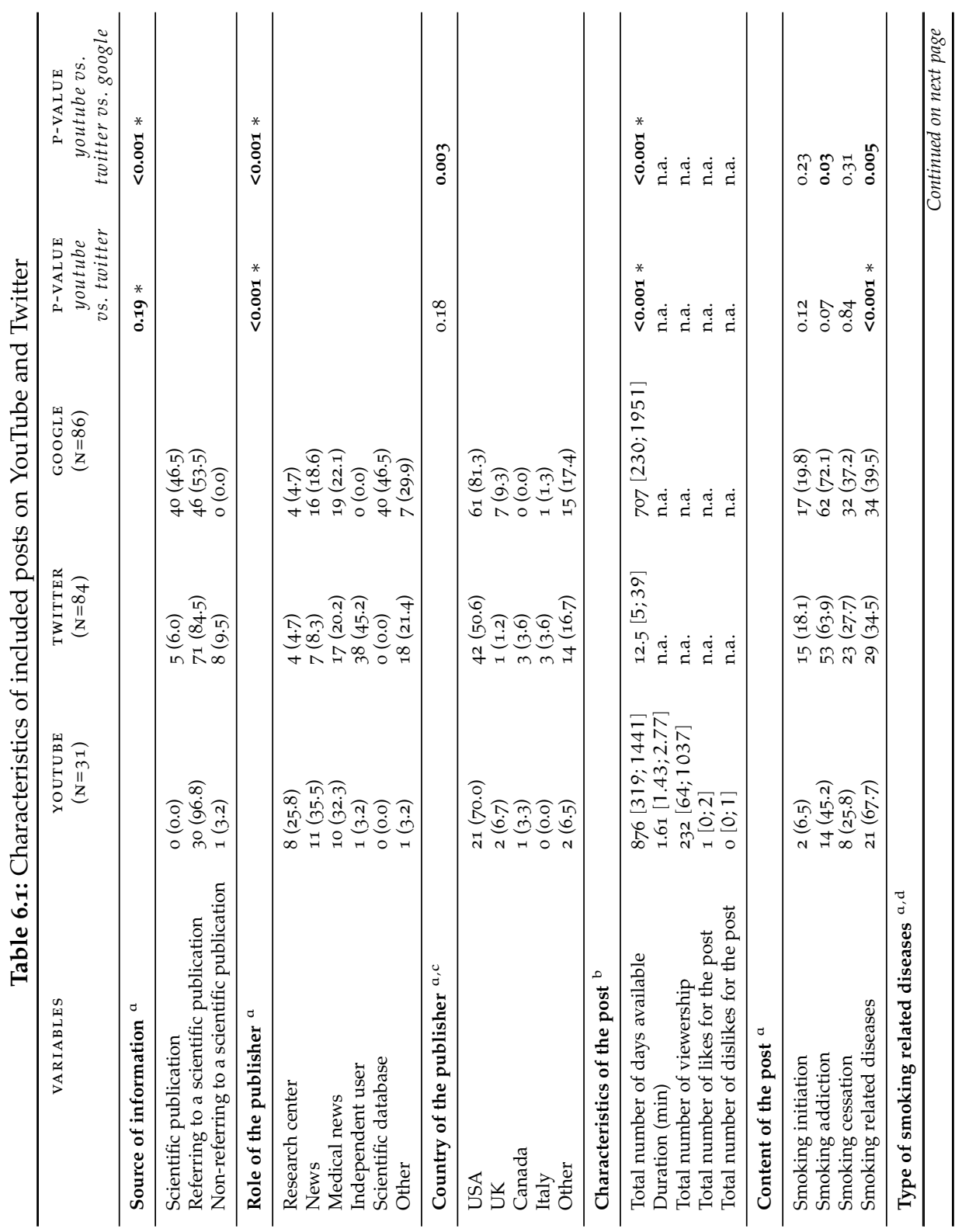


COMMUNICATING GENETICS AND SMOKING THROUGH SOCIAL MEDIA I IOI

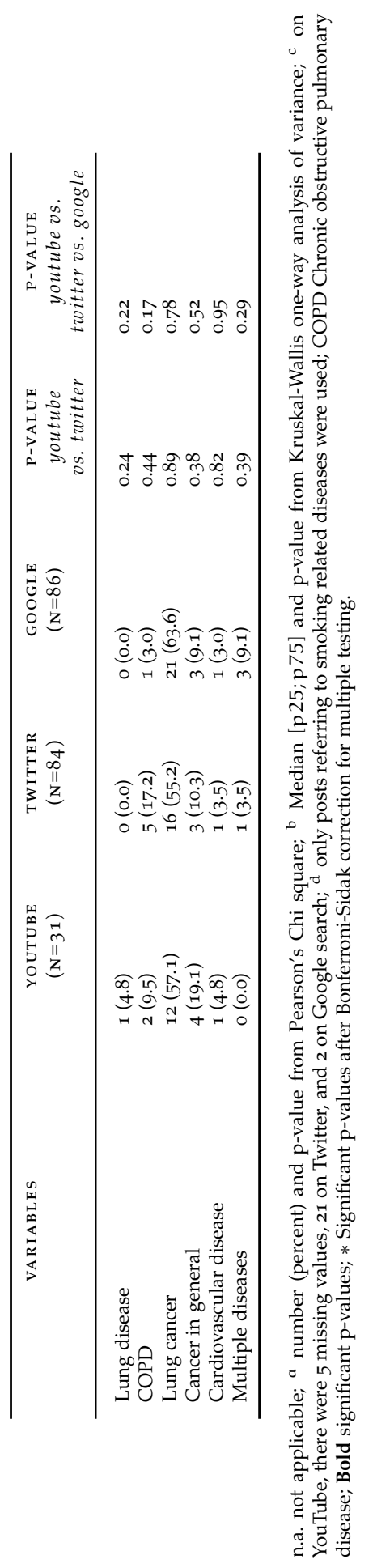


When comparing included posts obtained from Twitter and YouTube (Table 6.1), no differences in the source of information or in the country of the publisher were observed. However, the role of the publisher was significantly different between the two media $(P<0.001)$. Most publishers were independent users on Twitter $(45.2 \%)$ although it was the smallest role category $(3.2 \%)$ on YouTube. On YouTube, most posts were published by news or medical news instead of independent users on Twitter. For the content of the posts, a higher number of YouTube posts reported an impact of genetics on smoking related disease than on Twitter $(P=0.001)$. The other contents (smoking initiation, addiction and cessation) obtained similar results on YouTube and Twitter. For smoking related diseases, no comparison of the different types of disorders led to differences between YouTube and Twitter.

Between the two time points for both YouTube and Twitter, posts did not differ in the source of information, role of the publisher, country of the publisher, and characteristics of the posts. On YouTube, the content of the posts were not different between the two time points. By contrast, on Twitter, the content differed for smoking initiation $(P<0.001)$, addiction $(P<0.001)$, cessation $(P<0.001)$, and related disease $(P<0.001)$ (Table 6.2). Moreover, on Twitter, there was a significant difference in the number of days the posts were available $(P<0.001)$.

Table 6.2: Comparison of the content of the posts between Time 1 and 2

\begin{tabular}{|c|c|c|c|c|c|c|}
\hline & $\begin{array}{l}\text { youtube } \\
\text { Time } 1\end{array}$ & Time 2 & P-value & $\begin{array}{c}\text { TwITteR } \\
\text { Time } 1\end{array}$ & Time 2 & P-value \\
\hline Smoking initiation & o (o.o) & $2(22.2)$ & 0.02 & $\mathrm{o}(\mathrm{o.o})$ & $15(31.9)$ & $<0.001 *$ \\
\hline Smoking addiction & $8(36.4)$ & $6(66.7)$ & 0.12 & $13(36.1)$ & $40(85.1)$ & $<0.001 *$ \\
\hline Smoking cessation & $6(27 \cdot 3)$ & $2(22.2)$ & 0.77 & $1(2.8)$ & $22(46.8)$ & $<0.001 *$ \\
\hline Smoking related disease & $17(77 \cdot 3)$ & $4(44.4)$ & 0.08 & $22(61.1)$ & $7(14.6)$ & $<0.001 *$ \\
\hline
\end{tabular}

number (percent) and p-value from Pearson's Chi-square; Bold significant p-values; $*$ Significant p-values after Bonferroni-Sidak correction for multiple testing.

\section{Comparison between social media and Google search}

Of the first 100 websites retrieved from the Google search, 86 were related to genetics and smoking. No new social media channels were revealed from this search. Websites retrieved from Google search were different from the posts on YouTube and Twitter, both in source of information and role of publisher. On Google, websites were more often scientific publications $(46.5 \%)$ than on YouTube $(0.0 \%)$ or Twitter $(5.9 \%)$, explaining also the difference in the role of the publisher (scientific database, $46.5 \%$ ) (Table 6.1).

Some scientific publications referred to on YouTube were also found on the Google search (e.g. Amos et al. [287] was listed five times on YouTube and once on Google) and the same for Twitter (e.g. Govidan et al. [288] appeared 12 times on Twitter and 1 time on Google, and Belsky et al. [289], 38 times on Twitter and 9 on Google). However, no scientific publication found on YouTube was also retrieved on Twitter. 
Word clouds of the three social media and Google search

Further exploration of the Twitter posts and the post titles on YouTube and Google search through word clouds (Figure 6.3) showed that the words 'smoking', 'genetic' and 'cancer' were highly present. This result was in line with the high level of posts assessing smoking related diseases. On YouTube, the word 'Insidermedicine' was also highly reported. Over the 31 included posts, 6 were from Insidermedicine and summarised new studies published in scientific journals. The '2010' word in the graph is also due to Insidermedicine posts where the date of publication was written in the title. On Twitter, among others, the words 'lung', 'addiction' and 'teens' were frequently reported. This referred to two scientific publications that were reported multiple times; 12 Twitter posts (14.3\%) reported that smokers with lung cancer have tenfold genetic damage in comparison to never-smokers [288] and 38 posts $(45.2 \%)$ referred to the genetic factors influencing addiction in teens [289]. In the Google search, the words 'addiction', 'cessation' and 'risk' were most often used, giving an indication of the content of the websites.

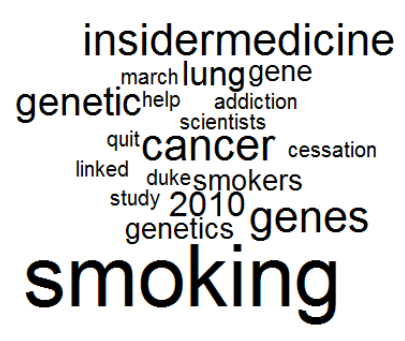

(a)

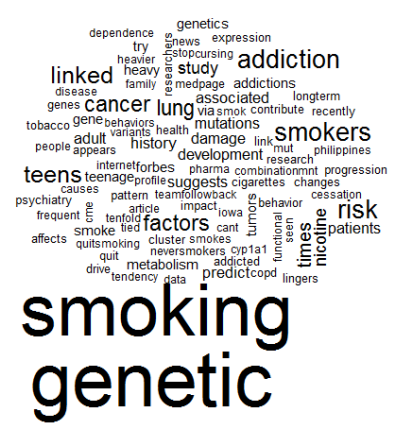

(b)

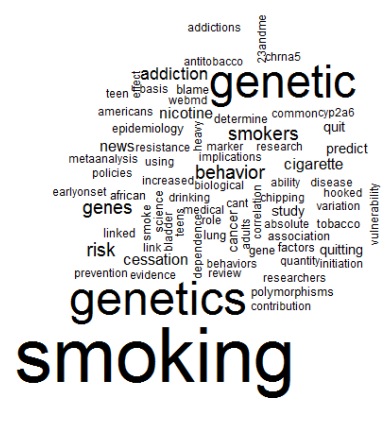

(c)

(a) presents the word cloud including the most used words in the title of YouTube posts; (b) the most used words from the posts on Twitter; (c) The most used words on Google. Each word's frequency is correlated with font size.

Figure 6.3: Word cloud presenting the most used words on YouTube, Twitter, and Google

\section{DISCUSSION}

The current study introduced the availability of information regarding genetics and smoking on three different social media (YouTube, Facebook and Twitter). This is, to our knowledge, the first study that investigated the availability of genetics and smoking on social media.

The results indicated that little information is available regarding the topic and even null on Facebook. The lack of posts on Facebook was not surprising given the specialised topic. However, we do expect the number of posts to grow dramat- 
ically as genetic testing and personalised medicine become more widely known. For YouTube and Twitter, most posts referred to scientific publications that were recently published.

The reason why no posts on genetics and smoking were retrieved using Facebook, versus the 472 ,ooo posts obtained by using the search 'smoking + genetic + site:facebook.com', is that Facebook is not a search engine and by contrast to Google is. Interestingly, the same search strategy in another search engine resulted in a different number of retrieved posts (e.g. using Bing.com 37,000 posts were found). Nonetheless, social media do not allow for such advanced search strategies and users do not typically use such search strings. Our objective was to know what information lay people find on genetics and smoking when using social media, so we developed our search within the social media and used typical search strategies.

The reason for the highest number of posts coming from the United States in comparison to other countries may be explained by the size of the country and high Web adoption rate [290], rather than the importance of the topic in any country. For example, Facebook had the highest number of users in the United States $(167,554,700$ users). Brazil was second with only 60,665,740 users. In comparison, the United Kingdom had 33,227,180 users and was in sixth position [291]. Despite the European ITFoM project (IT Future of Medicine) [292], PHGEN project (Public Health Genomics European Network) [4], the activities of the EAPM (European Alliance for Personalised Medicine) [293], and the high policy attention of it in Europe, the mere size of the continents and way in which each respective public accesses science information, probably helps to explain why the posts from Europe were fewer than in the USA.

On Twitter, the period of availability of the posts changed between Time 1 and 2 (from approximately one week to several months). This may explain the highest number posts included in Time 2.

The contents of the posts mostly focused on genetics of addiction and smoking related diseases. This result is consistent with the higher number of scientific publications on both topics than on any other topic linking genetics and smoking. The smoking related disease of interest was mainly lung cancer; this is also in line with the knowledge of the general population. Simon et al. reported that smoking as a risk factor for lung cancer was recalled by $85 \%$ of the population and recognised in a list of factors by $94 \%$ of the population [294].

The opinion about the posts on YouTube (measured by the number of likes and dislikes, as reported on Table 6.1) should be viewed cautiously due to the small number of observations. The range of 'like' posts was between o and 16 and 'dislike' between $o$ and 4 while the number of viewers ranged from 17 to 10,350. Moreover, for the posts that were retrieved in both time points, the number of likes and dislikes did not change significantly.

The difference between YouTube and Twitter for the number of days of a post's availability on the Web $(P<0.001)$ is explained by the fact that the Twitter search was performed by date and that posts on YouTube were classified by relevance. The 
search by date for Twitter and relevance for YouTube were used because they were the default options and therefore, probably the most frequently used by the population. Nevertheless, no differences between YouTube and Twitter were reported in the source of information and the country of the publisher, making the information comparable.

When using Google search as an indicator of the information about genetics and smoking available on the Web, we first observed differences between the posts retrieved on social media. As expected, most websites were scientific publications per se $(46.5 \%)$ or referred to scientific publications $(53.5 \%)$. Six scientific publications were referred at least once on the Google search and either on YouTube or Twitter $[68,287-289,295]$. The second objective of searching Google was to discover any other social media that included discussions of genetics and smoking. Despite our expectations, no social media, such as health forums or blogs, were retrieved. This might be explained by the novelty of the topic.

Information on genetic testing and smoking is already on the Web with direct-toconsumer testing such as 23andMe where they look for the CHRNA3 gene, more specifically the variant rsio51730. $\mathrm{CHRNA}_{3}$ is a nicotinic receptor. Tests on smoking related disorders are also available on 23andMe for lung cancer. As proposed by Pray in 2008, "imagine reading this warning on a cigarette package: Smokers with a particular mutation have a dramatically higher risk of developing lung cancer. Would you get tested for this mutation?" [296]. In the future, this kind of message may also be displayed on social media.

The way that individuals understand the posts on YouTube and Twitter should be assessed in a further study. Indeed, it is becoming more common that people are looking to different social media to get information about their health [279]. As public health genomics, personalised medicine, and personalised health terms become more commonly known terms, the public, including the general population, patients, and health professionals, will certainly look to social media to learn more about them and discuss them. Hence, information must be translated and communicated in a way for the general public to understand, especially since genome-based information, which includes genetic information, is a complicated topic for the nonscientific population.

Providing health information via social media and developing methods to evaluate their impact may help in effectively increasing health literacy and risk awareness in an innovative way, with attention to avoiding the introduction or widening of health inequalities. However, to generate effective health information messages, different conditions may be needed depending, for example, on the topic, target group, or society, taking into account both environment (internal and external) and the process of information (automatic and rational) [39]. Therefore, there is a real need to develop efficient communication tools to improve the health and genetic literacy of the population. Moreover, any message should aim to be correct, clear and adapted to the target population to maximise understanding of the content. Also, any ethical and legal issues of displaying such messages should be considered. Achieving 
these conditions is of critical importance to develop quality information that can be obtained and understood by those accessing it and those who need it.

Future research should examine the impact that information about genetics and smoking on popular social media has on target population literacy and behaviours. Exposure to genetic information about smoking in social media might be examined in various target populations (e.g. university students and pregnant women) in controlled settings where the target population is exposed to different genetic information about smoking during a certain period of time (e.g. one week). After the exposure step, the impact of the information and information channel on different outcomes (e.g. behaviour change, genetics and smoking knowledge) would be assessed. The exposure-outcome relationship might then be evaluated using advanced statistical methods, such as structural equation modeling. Finally, as with any communication channel, content spread through social media channels should be carefully scrutinised by the reader. All media have the potential to include biased and misleading information, but social media platforms can spread such information rapidly. At the same time, social media also allow for corrections and dialog about content to occur quickly and transparently. Moreover information may or may not be beneficial, but the ability to understand if the information is credible and ways in which to improve critical thinking and appraisal skills of social media users should be a priority of research and practice as well as codes of conduct for posting information.

\section{Limitations}

The most important limitation of our study is that we collected data from channels that change rapidly, at only two points in time. Consequently, the posts that were selected in our review may not be reflective of what is posted at another time. The collection of data at time points separated by 9 months, may give better insights of the evolution of posts over time. However, as the content posted on social media happens constantly, data collected over time points may yield different results. Particularly on Twitter, our results are likely to be different depending on the time of the search. For example, at Time 1, we observed that $35.2 \%$ of the posts were based on the lyrics of a song, which are likely to be ephemeral. At Time 2, only $16.0 \%$ of the posts referred to that song.

The search strategy may have resulted in posts being missed. We limited the search to two search terms ('genetic' and 'smoking') and the first hundred posts, which may not have captured all the relevant posts. However, the results obtained in our search provide a reasonable perspective of what someone interested in smoking and genetics would find on the topic on YouTube, Facebook and Twitter. Facebook, as a relatively closed system, did not allow an indeepth overview look at the posts of users.

The limits on the three selected social media may influence the obtained results. Other social media such as health forums might lead to different results. 


\section{Implications for practice and research}

This study focused on the availability of information on genetics and smoking and serves as a baseline measure from September 2012 and May 2013. Given the growing use of social media for health purposes, there is a need to monitor this situation over time to avoid the dispersion of false information. The topic of genetics and smoking is not currently widely discussed on the three social media platforms chosen. However, this is expected to change due to growing concerns about genetics in other media such as newspapers. This study did not provide any information on the profile of the viewers (e.g. smokers or non-smokers) or the use of that information (e.g. subsequent change in smoking behaviour). A future study assessing the habits and the characteristics of the population looking for health information (e.g. general population, patients, and health professionals) and more specifically information about genetics and smoking, will be needed. Moreover, a better overview of the users' understanding of the displayed information will be of high importance. Also, from the scientific point of view, the concept of 'genetic information' needs to be broadened towards 'genome-based information', taking into account emerging knowledge from the whole 'omics' field including epigenomics and the interaction of genomics and environment, such as in the case of smoking.

This study suggests that most of the information about genetics and smoking available on social media referred to scientific publications displayed by different kind of publishers (research center, news, and medical news). Increasing access to such information might improve the health and the genomic literacy of the population and, therefore, enhance smoking prevention and cessation. 

WHICH INTERNET TOOLS ARE SEARCHED BY STUDENTS AT UNIVERSITY TO OBTAIN INFORMATION ABOUT HEALTH, SMOKING AND GENETICS? A PILOT STUDY

Sylviane de Viron Angela Brand Servaas A. Morré Kasia Czabanowska Herman Van Oyen 
Objectives The Internet is a new tool that provides health information and therefore may enhance the health literacy of the population. The study aims were twofold, to explore what Internet tools are used and what factors influence the search of information about health, smoking and genetics among Belgian university students on the Internet.

Methods In this pilot study, 9 out of 14 conveniently selected faculties of the Catholic University of Louvain in Belgium sent out a web-based questionnaire based on the Information seeking behaviour model to their students. The questionnaire was available from the $1^{\text {st }}$ of March till the $31^{\text {st }}$ of May 2013. Factors assessed were general demographics, smoking behaviour, and the use of social media to obtain information about health, smoking and genetics. Statistical analyses included Chi-square, t-test, Spearman correlations and multivariate logistics regressions.

Results Out of 2,171 students who answered the full questionnaire, 1,937 were searching for information about health, smoking and/or genetics on Internet tools. Students were mainly searching for information about health, smoking and/or genetics on web search engine $(92.9 \%)$ and Forum $(44.6 \%)$. Factors influencing the search of information about health were monthly budget, ethnicity, smoking status and the faculty of study $(P<0.001)$.

Conclusions University students are already used to searching for information about health, smoking and genetics on different Internet tools. Health topics are more frequently questioned than smoking and genetics. Therefore, monitoring and developing targeted messages on such media may improve the health and genetic literacy. 


\section{INTRODUCTION}

With the growing access to the Internet and the ability to provide, gather and share information, Internet tools became a new source of health information. Indeed, the Internet is an easy tool allowing dissemination of health information to a large population in an inexpensive way [297]. Moreover, in comparison to other media such as television or newspaper, it allows the immediate information access [298]. Information may be gathered in an anonymous way and tailored for specific populations of interest (e.g. young adults and pregnant women) [299].

In 2010 , about $80 \%$ of the American population connected to the Internet was searching for health information on the Internet [30o]. The Internet is used for different purposes such as buying medicine and vitamins, communicating with physicians and participating in group discussion about medical conditions [301]. Genetics, as a field in huge expansion, represents new kinds of information available on the Internet. The use of genetic information leads to more personalized healthcare by, for example, communicating genetic risk of diseases or personalizing treatment based on genetic criteria. Direct-to-consumer genetic testing were read or heard by $29.2 \%$ of the population connected to the Internet in an American survey and around $8.1 \%$ bought the genetic test [301]. Despite the popularity of searching for health information on the Internet, people are still going to their physicians for confirmation or affirmation of what they searched [301, 302].

Research indicates that online communication is effective at improving health literacy of the population on specific health topics. Moreover, it appears to induce effective behaviour change that may have an impact on short and long-term followup. The use of theory (e.g. theory of planned behaviour), behaviour change techniques (e.g. emotional control setting) and methods of interaction with participants appear to improve the effectiveness of the online intervention [303].

The most important challenge facing the Internet tools, which serve as a source of health information, is to make Internet users access and return to a specific Internet page. To enhance this point, rapidly evolving and dynamic content might be adopted. Moreover, promoting active users is also a positive factor [299].

However, some concerns regarding the display of health information on the Internet are evidenced. They included questions about the quality of the information, the quantity of the information that may lead to confusion and reduced trust in physicians [39]. Moreover, a recent study assessed the availability of genetic information about smoking on three different social media (Twitter, Facebook and Twitter). The available information mostly referred to scientifically based publications [304]. Therefore, there is a need to assess the type of Internet tools used by the population, to develop new health information on Internet tools and to monitor the quality and the quantity of the information displayed. Indeed, improving health information on the Internet might enhance the health literacy of the population [254].

Given the importance of smoking as a major public health problem and the novelty of the genetics field, these two specific areas were used in combination with 
health to have a broader idea of the state of the search on health information related to Internet tools. The aim of the study is twofold: to explore what Internet tools are used and what factors influence the search of information about health, smoking and genetics among Belgian university students on the Internet.

\section{METHODS}

\section{Study design and study population}

A cross-sectional study was conducted between the $1^{\text {st }}$ of March and the $31^{\text {st }}$ of May 2013 among students registered in 9 conveniently selected faculties out of the 14 faculties of the UCL. The 9 included Faculties were: law; polytechnic; political, economic and social science; pharmacy and biomedical science; philosophy, arts and letter; motor functions science; public health; architecture; psychology and education. The UCL is the largest French university in Belgium including more than 28, ooo students. To get a higher visibility and therefore a potentially higher response rate, students got informed about the present study by a single email sent by the Dean of the Faculty. The Deans sent a single email to their students.

\section{Questionnaire}

A web-based questionnaire was formatted using LimeSurvey. LimeSurvey is an open source tool for online surveys that allows the storage of the answers on personal servers. The questionnaire was based on the conceptual frameworks of Johnson et al. [305] and Niedwiedzka et al. [306]. The questionnaire was developed in French and included 37 questions arranged in four groups: (i) socio-demographics, (ii) tobacco consumption, (iii) opinion and knowledge about tobacco and genetics, and (iv) the search of information about health smoking and genetics on Internet tools. Questions were either multiple choice, true-false or 5-point Likert scale questions. The questionnaire was pre-tested on a sample of 10 university students, allowing the adaptation of questions. The adapted questionnaire was used in the present study.

\section{Data and measures}

Only fully answered questionnaires were used in the calculations. However, as some questions were not mandatory or followed a filter the sample size may differ for some analyses.

Socio-demographic questions were used to control the statistical calculations. For ethnicity, three options were available: Belgian (coded as o), European excluding Belgian (coded as 1) and non-European (coded as 2). Variables about education (bachelor, master and doctoral students) and the Faculty of study were extracted from one single open-question. The measure of monthly budget was an average of 
the monthly net residual income including both pocket money and money earned by working and excluding the amount of money used to pay the housing. Seven options were proposed: less than 50 euros (coded as 1); between 50 and 99 euros (coded as 2); between 100 and 199 euros (coded as 3); between 200 and 399 euros (coded as 4); between 400 and 599 euros (coded as 5); between 600 and 899 euros (coded as 6); more than 899 euros (coded as 7).

The smoking status, as self-reported by students, was separated in the following categories: never smoker (coded as o), former smoker (coded as 1), occasional smoker (not smoking every day) (coded as 2) and current smoker (smoking every day) (coded as 3). The score of genetic knowledge, ranging from o to 3, was calculated based on the three following true/false questions: Each individual, carrier of a genetic disorder whichever it is, will develop the symptoms of the disorder (False); Most human characteristics are controlled by a single gene (False); A cell from a nail contains all genetic information necessary to create a human (True). Each right answer increased the score by one.

Internet tools used by students were assessed for the three topics (health, smoking and genetics) using the following options: no tool; web-search engine (e. g. Google); Forum (e.g. Doctissimo); Facebook; Twitter; YouTube. Moreover, students had the opportunity to report other Internet tools. The frequency in the use of social media was evaluated with the following options: never (coded as o), less than once a year (coded as 1), more than once a year (coded as 2); more than once a month (coded as 3), more than once a week (coded as 4 ), and more than once a day (coded as 5 ).

\section{Outcome variables}

The three different outcomes variables were searching for information about health, smoking and genetics using Internet tools. The outcomes were dichotomous (Yes or No, respectively coded as 1 and o).

\section{Statistical analyses}

Univariate analyses were assessed by the Pearson's Chi square for categorical data and the t-test for continuous data. Spearman correlations were developed using Bonferroni correction for multiple testing.

Stepwise logistic regression models were used to determine explanatory variables of searching for information about health, smoking and genetics on different types of Internet tools. For each of the model, a forward and a backward selection were conducted, using a significance level of, respectively, 0.05 and 0.10 . Independent variables were selected if the correlation with the outcome was higher than 0.05 . This method was used due to the low current knowledge of factors influencing the search of information about health, smoking and genetics on Internet tools. Moreover, interactions between reasons to quit smoking and other variables were examined. 
Analyses were two-sided with a significance rate $\alpha$ of 0.05 . All statistical analyses were performed using STATA, version 10.1 (STATA Corporation Inc., College Station, TX, USA).

\section{RESULTS}

Initially 2,574 students answered the questionnaire, 403 answers were removed due to (i) incomplete questionnaire, (ii) inconsistent answers and (iii) not being a student at the UCL or from one of the nine selected Faculties. The final sample included 2,171 participants (Figure 7.1), which represents about $10 \%$ response rate of the 9 included Faculties (the same response rate was observed in each Faculty separately). As in the total population of students from the UCL, the mean age was about 22 years and women were more represented (63.9\%). Most students were actually doing their bachelor studies $(55.2 \%)$ and living in student housing (49.4\%). About $12.8 \%$ of students were currently smoking and $11.4 \%$ occasional smokers. Moreover, most students had the maximum score of genetic knowledge (59.9\%). Additionally, students mostly used social media more than once a day (79.2\%) (Table 7.1).

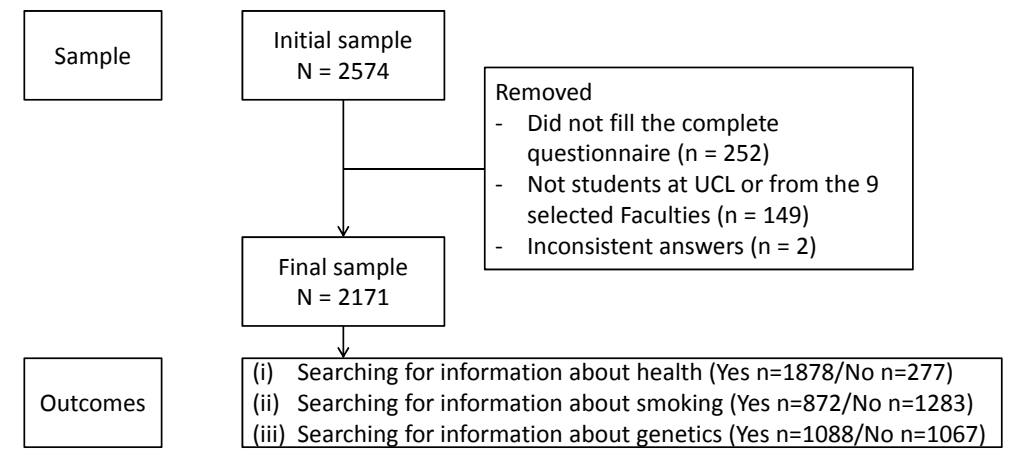

Figure 7.1: Flow diagram of data of the study exploring the Internet tools used by students at the Catholic University of Louvain to search for information about health, smoking and genetics

Most students were searching for information about at least health, smoking or genetics on Internet tools $(89.2 \%)$. Those students searching for information on Internet tools were mostly women $(P<0.001)$, living in student housing $(P=0.003)$ and using social media more than once a day $(P<0.001)$ (Table 7.1).

Among different Internet tools, students were mainly searching for information about health on web-search engine $(78.7 \%)$; this result was observed in a lower case for tobacco $(34.4 \%)$ and genetics $(45.0 \%)$. Web-search engine was followed by, respectively, Forum, YouTube, Facebook and Twitter. Moreover, students were more frequently using no tools to search for information about smoking (59.5\%) or genetics $(49.5 \%)$ than health (12.9\%) (Table 7.2$)$. 
Table 7.1: General characteristics of the student's population by searching or not for information about at least health, smoking or genetics in Internet tools

\begin{tabular}{|c|c|c|c|c|}
\hline & $\begin{array}{c}\text { SEARCHING } \\
\text { FOR INFORMATION } \\
(\mathrm{n}=1932)\end{array}$ & $\begin{array}{l}\text { NOT SEARCHING } \\
\text { FOR INFORMATION } \\
(\mathrm{n}=239)\end{array}$ & $\begin{array}{c}\text { TOTAL } \\
(\mathrm{N}=2171) \\
\end{array}$ & P-VALUE \\
\hline Male gender ${ }^{a}$ & $658(34.1)$ & $126(52.7)$ & $784(36.1)$ & $<0.001$ \\
\hline Age $^{b}$ & $21.8 \pm 3.8$ & $21.8 \pm 4.6$ & $21.8 \pm 3.9$ & 0.95 \\
\hline Ethnicity ${ }^{a}$ & & & & 0.37 \\
\hline $\begin{array}{r}\text { Belgian } \\
\text { European } \\
\text { Non-European }\end{array}$ & $\begin{array}{c}1687(87.3) \\
190(9.8) \\
49(2.5)\end{array}$ & $\begin{array}{c}209(87.5) \\
25(10.5) \\
3(1.3)\end{array}$ & $\begin{array}{c}1896(87.3) \\
215(9.9) \\
52(2.4)\end{array}$ & \\
\hline Monthly budget a & & & & 0.40 \\
\hline $\begin{array}{r}\text { Less than } 50 \text { euros } \\
\text { Between } 50 \text { and } 99 \text { euros } \\
\text { Between } 100 \text { and } 199 \text { euros } \\
\text { Between } 200 \text { and } 399 \text { euros } \\
\text { Between } 400 \text { and } 599 \text { euros } \\
\text { Between } 600 \text { and } 899 \text { euros } \\
\text { More than } 899 \text { euros }\end{array}$ & $\begin{array}{l}186(9.8) \\
333(17.6) \\
522(27.6) \\
561(29.7) \\
141(7.5) \\
34(1.8) \\
87(4.6)\end{array}$ & $\begin{array}{l}30(13.2) \\
48(21.1) \\
56(24.6) \\
58(25.4) \\
21(9.2) \\
3(1.3) \\
8(3.5)\end{array}$ & $\begin{array}{l}216(10.2) \\
381(18.0) \\
578(27.3) \\
619(29.2) \\
162(7.7) \\
37(1.8) \\
95(4.5)\end{array}$ & \\
\hline Education $^{a}$ & & & & 0.76 \\
\hline $\begin{array}{l}\text { Bachelor students } \\
\text { Master students } \\
\text { Doctoral students }\end{array}$ & $\begin{array}{c}1026(55.0) \\
766(41.0) \\
19(1.0)\end{array}$ & $\begin{array}{c}131(57.5) \\
90(39.5) \\
1(0.4)\end{array}$ & $\begin{array}{c}1157(55.2) \\
856(40.9) \\
20(1.0)\end{array}$ & \\
\hline Faculty ${ }^{a}$ & & & & 0.14 \\
\hline $\begin{array}{r}\text { Law } \\
\text { Polytechnic } \\
\text { Political, economic, and social science } \\
\text { Pharmacy and biomedical science } \\
\text { Philosophy, arts and letter } \\
\text { Motor functions science } \\
\text { Public health } \\
\text { Architecture } \\
\text { Psychology and education }\end{array}$ & $\begin{array}{l}246(12.7) \\
223(11.5) \\
692(35.8) \\
104(5.4) \\
280(14.5) \\
120(6.2) \\
56(2.9) \\
96(5.0) \\
115(6.0)\end{array}$ & $\begin{array}{c}29(12.1) \\
39(16.3) \\
93(38.9) \\
9(3.8) \\
30(12.6) \\
11(4.6) \\
3(1.3) \\
16(6.7) \\
9(3.8)\end{array}$ & $\begin{array}{c}275(12.7) \\
262(12.1) \\
785(36.2) \\
113(5.2) \\
310(14.2) \\
131(6.0) \\
59(2.7) \\
112(5.2) \\
124(5.7)\end{array}$ & \\
\hline Smoking status ${ }^{a}$ & & & & 0.06 \\
\hline $\begin{array}{r}\text { Never smoker } \\
\text { Former smoker } \\
\text { Occasional smoker } \\
\text { Current smoker }\end{array}$ & $\begin{array}{l}1366(70.7) \\
110(5.7) \\
222(11.5) \\
234(12.1)\end{array}$ & $\begin{array}{l}154(64.4) \\
16(6.7) \\
26(10.9) \\
43(18.0)\end{array}$ & $\begin{array}{l}1520(70.0) \\
126(5.8) \\
248(11.4) \\
277(12.8)\end{array}$ & \\
\hline Use of social media ${ }^{a}$ & & & & $<0.001$ \\
\hline $\begin{array}{r}\text { Never } \\
<1 \text { time per year } \\
>1 \text { time per year } \\
>1 \text { time per month } \\
>1 \text { time per week } \\
>1 \text { time per day }\end{array}$ & $\begin{array}{c}10(0.5) \\
7(0.4) \\
22(1.1) \\
34(1.8) \\
299(15.5) \\
1560(80.8)\end{array}$ & $\begin{array}{c}16(6.7) \\
1(0.4) \\
2(0.8) \\
5(2.1) \\
55(23.0) \\
160(67.0)\end{array}$ & $\begin{array}{c}26(1.2) \\
8(0.4) \\
24(1.1) \\
38(1.8) \\
354(16.3) \\
1720(79.2)\end{array}$ & \\
\hline Genetic knowledge score $^{a}$ & & & & 0.39 \\
\hline $\begin{array}{l}0 \\
1 \\
2 \\
3\end{array}$ & $\begin{array}{c}3(0.2) \\
97(7 \cdot 3) \\
426(32.0) \\
804(60.5)\end{array}$ & $\begin{array}{c}1(0.7) \\
15(9.7) \\
52(34.8) \\
84(54.8)\end{array}$ & $\begin{array}{c}4(0.3) \\
112(7.5) \\
480(32.3) \\
889(59.9)\end{array}$ & \\
\hline
\end{tabular}

$\overline{\mathrm{a}}$ number (percent) and p-value from Pearson's Chi-square; ${ }^{\mathrm{b}}$ mean \pm standard deviation and p-value from t-test; Bold significant $\mathrm{p}$-values 
Multivariate models of explanatory variables of the search for information about health, smoking and genetics are reported in Table 7.3. The two Internet tools included and combined in the model were web-search engine and Forum. Predictors of searching for information about health were monthly budget, ethnicity, faculty of study and smoking status. Those predictors explained $77.2 \%$ of the variability of searching for information about health $\left(\mathrm{LR} \mathrm{Chi}^{2}=1195.1, P<0.001\right)$. In smoking, $75.1 \%$ of the variability of searching for information about smoking was explained by budget and the Faculty of study. Web-search engine and Forum, as Internet tool, were included in the model $\left(\mathrm{LR} \mathrm{Chi}^{2}=1,669.8, P<0.001\right)$. The last model assessing the search of information about genetics included budget and web-search engine as Internet tool (LR Chi $\left.{ }^{2}=885.7, P<0.001\right)$.

Table 7.2: Types of internet-based tools used to obtain information about health, smoking and genetics

\begin{tabular}{rccc}
\hline & HEALTH & SMOKING & GENETICS \\
\hline No tool & $277(12.9)$ & $1283(59.5)$ & $1067(49.5)$ \\
Web-search engine & $1696(78.7)$ & $741(34.4)$ & $970(45.0)$ \\
Forum & $837(38.8)$ & $192(8.9)$ & $190(8.8)$ \\
Facebook & $32(1.5)$ & $14(0.7)$ & $9(0.4)$ \\
Twitter & $7(0.3)$ & $3(0.1)$ & $3(0.1)$ \\
YouTube & $72(3.4)$ & $40(1.9)$ & $51(2.4)$ \\
\hline \multicolumn{5}{c}{ number (percent) }
\end{tabular}

Table 7.3: Multivariate logistic regressions about the use of Internet tool to search for information about health, smoking and genetics

\begin{tabular}{|c|c|c|c|}
\hline VARIABLES & $\begin{array}{l}\text { HEALTH } \\
(\mathrm{n}=2012)\end{array}$ & $\begin{array}{l}\text { SMOKING } \\
(\mathrm{n}=1660)\end{array}$ & $\begin{array}{l}\text { GENETICS } \\
(\mathrm{n}=908)\end{array}$ \\
\hline Monthly budget ${ }^{a}$ & $1.38(1.13-1.68)$ & $1.31(1.13-1.53)$ & $1.31(1.04-1.64)$ \\
\hline Ethnicity ${ }^{a}$ & $1.93(0.99-3.75)$ & & \\
\hline Faculty ${ }^{a}$ & $1.13(1.00-1.29)$ & $1.11(1.00-1.22)$ & \\
\hline Smoking status a & 0.79 (0.61-1.01) & & \\
\hline \multirow{2}{*}{\multicolumn{4}{|c|}{$\begin{array}{l}\text { Searching for information } \\
\text { on web-search engine } a, b\end{array}$}} \\
\hline & $827.16(358.20-1910.07)$ & $1300.62(550.93-3070.54)$ & $706.42(294 \cdot 36-1695 \cdot 32)$ \\
\hline & $266.54(78.94-899.92)$ & $122.24(34 \cdot 57-432.22)$ & \\
\hline$L R C h i^{2}$ & 1195.1 & 1669.8 & 885.7 \\
\hline P-value & $<0.001$ & $<0.001$ & $<0.001$ \\
\hline Pseudo $R^{2}$ & $77.2 \%$ & $75.1 \%$ & $70.6 \%$ \\
\hline
\end{tabular}

a Odds ratio (95\% confidence interval); ${ }^{b}$ Search for information about health in the health model, smoking in the smoking model and genetics in the genetic model.

\section{DISCUSSION}

This is, to our knowledge, the first study that explored the types of Internet tools and different factors influencing the search of information about health, smoking and genetics on the Internet. 
Results indicated that Internet tools were mainly used to search for information about health and in a lower case about smoking and genetics. A previous study reported that around $60 \%$ of the population searched for health information at least once a month. The topic of health information search was mostly symptoms, treatment and diseases/conditions [302, 307]. Moreover, people were searching for health information for themselves $(37 \%)$, for someone else $(49 \%)$ and both $(8 \%)$ [307].

Regarding the Internet tools used, the dominance of web-search engine to search for information about health, smoking and genetics is in line with results reported by Elkin et al. [302]. Web-search engine gained the trust of a large audience. Moreover, they reported an emergence of search on social media including e. g. Wikipedia, Facebook or YouTube [302]. In the present study, the emergence of search on social media was mainly observed in Forum followed by YouTube, Facebook and lastly Twitter.

Factors explaining the search for information about health, smoking and genetics on Internet tools were gender, smoking status and the use of social media in univariate analyses and monthly budget, ethnicity, Faculty, and smoking status in multivariate analyses. These results are in line with those find in the literature $[298,302,307,308]$. Women were already reported to have an increased use of Internet tools to search health information in comparison to men [302]. Cotten et al. reported that age, education and income appeared to influence the search of health information on the Internet. Internet seekers were younger, with a higher degree of education and with higher incomes than non-seekers [298]. Additionally, Chou et al. reported that education, race and healthcare access did not have an impact on social media use in the context of health [308]. It is why, the current study targeted a population of university students inducing a more homoogeneous population in age but also education and income.

The reason why the smoking status variable was not included in the multivariate model of searching for information about smoking and the score of genetic knowledge variable in the multivariate model of searching for information about genetics remains open to interpretation. Either these two variables are truly not influencing the outcomes or they might be avoided due to power issues.

In the future, a better monitoring of the information about health, smoking, genetics and all other health topics displayed on Internet tools might be developed. Moreover, methods to improve translation from evidence-based health information including or not genetic information is of high importance to improve the health [307] and the genetic literacy of the population [254]. To develop such methods, first questionnaires assessing the types of preferred Internet tools (e.g. Forum or YouTube), the types of relied publisher (e. g. scientific publisher or peer), the types of preferred message (e.g. paternalistic or autonomy) and disorders of interest might be sent to targeted populations. Moreover, to improve the content of the message, questions might measure the knowledge, the understanding and the attention of the population to that type of message. Thereafter, the effectiveness of the developed 
messages might be evaluated in real life. The assessment of the effectiveness will be an important challenge due to the fact that there is no control on who actually search for the information.

\section{Limitations}

The present study, as a pilot study, did not aim to be representative of the total population of university students. Other studies will be needed in different universities to be able to generalise the results. However, even if the study was a pilot, a relatively high response rate was obtained (10\%) when knowing that students received only one email presenting the study. This response rate was comparable with studies assessing the effect of invitation on response rates, e. g. Kaplowitz et al. reported a response rate of $15 \%$ when sending two emails to the target population [309].

In multivariate analyses, the wide confidence intervals of Internet tools (websearch engine and Forum) to search for information about health, smoking and genetics (Table 7.3) indicated a limited precision of the odds ratios, likely due to the small sample size or residual confounding by unmeasured factors. Indeed the addition of interaction factors did not improve the confidence interval width.

Due to the cross-sectional design of our study, there might be recall biases. This might have an impact on the estimation. Nevertheless, the use of Internet tools to search for information about health is already suggested in different studies [298, 300, 302]. Future prospective studies should be able to control for the recall bias.

\section{Implications for practice and research}

Internet tools, as new sources for health communication, might be used in public health and health promotion to improve the health and the genetic literacy of the population. This kind of tools for health information should help, in a further step, in enhancing behaviour change. Due to the important search of health information on Internet tools and more specifically on web-search engine and forum, future research might monitor and develop adapted health messages for the population. To obtain faster translation from the scientific background to the population, methods of translation should be developed. Moreover, the effectiveness of such message must be further assessed in real life. 


GENERAL DISCUSSION 
Public Health Genomics (PHG), defined as 'the responsible and effective translation of genome-based knowledge and technologies into public policy and health services for the benefit of population health' $[1,2]$, is the field of translational research from basic sciences into healthcare systems, which give new insights in the prevention and the treatment of complex disorders and traits such as smoking.

Despite the widespread knowledge of consequences of smoking on health, smoking still remains a major public health problem worldwide. Indeed, tobacco killed around 6 million people worldwide from direct use or second-hand smoking in 2011 [19]. Moreover, it is an important risk factor for the four leading non-communicable diseases (cardiovascular diseases, diabetes, cancers, and chronic respiratory disorders) [19].

Like all complex disorders and behaviours, smoking is a multifactorial trait including both non-genomic and genomic factors. Genomic factors seem to be an important part of the causal mechanisms leading to smoking addiction and influencing smoking cessation. This is why, by improving the translation from bench to bedside and beyond to healthcare, PHG highly contributes to all health interventions tackling smoking.

Therefore, approaches and methods are needed to enhance the translation from bench to bedside and even beyond healthcare. Multiple approaches and methods are already developed such as the PHG enterprise (Figure 1.1) [1, 2], which is a tool that represents a way of approaching problems. It includes multiple factors necessary in the translation to the healthcare system, such as the role of genome-based science and technology and knowledge integration. Moreover, the wheel of public health (Figure 1.2) [10] is also an effective tool including different dimensions: assessment, policy development and assurance. Lal et al. [16] proposed a model to enhance translation using both technology transfer activity and public health assessment technologies in relationship to public-private partnerships. Khoury et al. [3] proposed a framework of translation aiming to move promising genomic applications to clinical and public health practice by using four different phases (from gene discovery to population health impact). Compared to LAL model, the Khoury framework does not include the technology transfer. These approaches and methods might be applied to all types of disorders and traits to enhance the translation of genomic research that is most often slow. For example, currently in smoking, no genomic applications are already in use in the daily clinical practice. However, in the near future, we could imagine to personalise treatment (in the type of drugs, dose, and duration) of smokers attempting to quit depending on their genes.

In the present thesis, we investigated innovative tools to enhance the translation of smoking from basic sciences into healthcare systems. For this, we explored and developed new tools to improve healthcare for smokers by taking both genomic and non-genomic factors into account. Furthermore, we investigated original ways to communicate genome-based information about smoking through genetic risk notification, the use of social media, and other Internet tools. Indeed, the Internet is an innovative medium for health information provision, gathering and transfer. 
This final chapter gives an overview of the main findings, the limitations, and implications for public health practice and future research for each of the two parts of the thesis (i) improving healthcare for smokers, and (ii) enhancing the communication of genomic risks about smoking in the general population. Thereafter, a final conclusion is formulated.

IMPROVING HEALTHCARE FOR SMOKERS

\section{Overview of the main findings}

In order to improve the health for smokers and healthcare interventions related to smoking, we first reviewed genetic predictors for smoking initiation and cessation (Chapter 2). Two main pathways of interest were associated with smoking initiation and cessation: (i) the cascade theory of reward (influencing the pharmacodynamics of nicotine), and (ii) the nicotine metabolism (influencing the pharmacokinetics of nicotine). The cascade theory of reward includes four main neurotransmitters: serotonin, opioid receptors, gamma-aminobutyric acid (GABA), and dopamine. Serotoninergic neurons release serotonin $(5-\mathrm{HT})$. This activates opioidergic neurons that, at the same time, release endogenous opioid. The opioid inhibits the release of GABA, and finally this inhibition increases the release of dopamine in the nucleus accumbens in the brain [26]. Genes influencing nicotine metabolism mostly include cytochrome $\mathrm{P}_{450}$ genes (e.g. $\mathrm{CY}_{2}$ B6). Moreover, nicotinic acetylcholine receptors are also of high importance in predicting smoking initiation and cessation. It includes two kinds of subunits: alpha $\left(\alpha_{1}-\alpha_{1}\right)$ ) and beta $\left(\beta_{2}-\beta_{4}\right)$. Genetic predictors of smoking cessation were found to interact with interventions used in smoking cessation (e.g. DRD2 Taq1A and bupropion) [73-75] and with other genes (DRD2-141C with FREQ rs1054879 and SLC6A3 with DRD2 Taq1A) [86, 87].

In Chapter 3, a systematic review of reviews of environmental factors, genomic factors and interventions influencing smoking cessation in the general population was carried out. This review reported eight categories of factors influencing smoking cessation: smoking behaviour (e.g. past quit attempts), demography (e.g. gender), socioeconomic status (e.g. income), social (e.g. marital status), health (e.g. health status), biology (e.g. menstrual phase), psychology (e. g. self-efficacy), and genomics (e.g. reward cascade). No factor presented a predominant effect on smoking cessation in previous meta-analyses. Relative risk ratios and odds ratios reported in meta-analyses ranged from 1.26 to 2.56 . These factors were dynamically linked to interventions, as presented by three target levels (individual, neighbourhood and society), in order to lead to smoking cessation as presented in the developed working model of smoking cessation (Figure 3.2). Factors included in the working model of smoking cessation were quite similar to the ones used in the integrative model of tobacco use and dependence developed by Swan et al. in 2002 [112], except intervention factors in the latter model. 
Based on a literature review and data-mining analyses, 32 genes influencing tobacco use disorder (TUD) were selected in Chapter 4. TUD, as defined by the Medical Subject Heading (MeSH) index, is the 'Tobacco used to the detriment of a person's health or social functioning. Tobacco dependence is included'. Most selected genes were nicotinic receptors. However, the cascade theory of reward and nicotine metabolism were well represented. Disorders associated with these genes were selected through data-mining and represented all expected disease categories (addiction, mental disorders, cardiovascular disorders, and cancer). When observing genetic similarities between selected disorders, two main clusters of disorders were observed: the first one including coexisting disorders of smoking (e.g. alcoholism and depressive disorder) and the second one, disorders that are side-effects of smoking (e.g. stroke and oesophageal cancer). This raises the question about the relationship between TUD and disorders. The relationship may either be due to causality, common pathways in systems medicine, or pleiotropy. The field of systems medicine takes into account multiple interactions, such as those retrieved in epigenomics. However, pleiotropy occurs when a single gene variant influences multiple phenotypic traits [310]. Nevertheless, genetics is only one part of the mechanism explaining the association between TUD and other disorders. Among other environmental and genomic factors, epigenomics and exposomics are plausibly influencing the association between TUD and other disorders.

\section{Limitations}

In both systematic reviews of Chapter 2 and 3, inclusion criteria might have introduced limitations in our results. We focused on prospective studies (except for the systematic review on smoking initiation) to reduce recall bias and for their better accuracy in giving information about causality. Nevertheless, this might have narrowed our obtained results due to the lack of publications. In Chapter 3 , restriction on prospective studies including at least 6-month follow-up even increased this limitation. However, most smoking relapses take place during the first six months after quitting [311]. Therefore, factors influencing smoking cessation might differ depending on the time to follow-up after quitting [311]. For example, self-efficacy and frequency of urges to smoke were related to smoking relapse only during the first month of cessation [311].

In Chapter 4, the search strategy to collect both genes and disorders associated to TUD might induce limitations in the study. Indeed, the search on Gene2MeSH, limited the review to publications available on PubMed and Ingenuity Pathway Analysis (IPA) uses data-mining as sources of information. It includes various source of information such as text-mining research and multiple databases. However, datamining may introduce false positivity because it does not take into account the true biological relation between elements. Moreover, genetics is only one part of the mechanism leading to both coexisting disorders of smoking and disorders that are side-effects of smoking. Therefore, further studies will have to include epigenomic 
factors to improve the understanding of the mechanisms linking TUD to other disorders.

\section{Implications for clinical practice and future research}

In order to improve healthcare for smokers, different strategies were proposed in Chapter 2, 3, 4. Understanding smoking in a holistic way including both environmental and genomic factors is essential to enhance smoking prevention and smoking cessation. Moreover, personalised interventions through, for example, individualised interventions for addicted smokers using pharmacogenetic tests to target the most effective and efficient drug, dose and duration based on multiple genetic variants are major approaches to improve healthcare for smokers.

In direct link to the work developed in the present thesis, a statistical validation of the working model of smoking cessation developed in Chapter 3 is needed. This validation should assess the interactions between the different factors and the effect size of each factor. A way to validate this model would be to develop a prospective cohort of smokers that are willing to quit smoking and to follow them for at least 6 months after quitting. The statistical technique to deal with this is structural equation modelling, in which the number of observations is based on the number of variables. In the proposed working model, around 40 variables are included, which implies that the minimum number of observations should be 820 . The strength of that kind of model would be that it allows to evaluate the effect of each single factor and consequently, to quantify the effect of the components [108]. Model testing through simulations under different conditions will also help in understanding complex systems behaving in an unexpected fashion [107].

In the future, nicotine might not be the only target for therapeutic interventions, but researcher will have to include multiple other chemicals (xenobiotica) contained in the cigarette. Deeper evaluation of epigenetic factors leading to behaviour change and how to communicate them to the general population are needed. Future research is required to better understand the biological mechanisms leading to smoking addiction and smoking related disorder. This better understanding will help in developing prediction models of smoking initiation, addiction, cessation or smoking related disease risks.

Moreover, the analyses developed in Chapter 4 might be extended to individual characteristics, effects of treatment, or severity of treatment. Analyses of the most significant disorders associated to TUD in micro-array online databases may highlight whether the expression of the genes are similar or different between disorders. Furthermore, experiments should be developed to highlight the mechanisms leading to the association between TUD and other disorders. This might be developed in either twin studies or prospective studies. In those studies, not only genetic factors might be analysed but also environmental and genomic factors.

Finally, in the present thesis, the target population was the general population. Therefore, applications of the research developed on Chapter 2, 3 and 4 on specific 
populations (e.g. pregnant women, multi-addict population, or population with a cardiovascular disease) might provide additional knowledge.

ENHANCING THE COMMUNICATION OF GENOMIC RISKS ABOUT SMOKING IN THE GENERAL POPULATION

\section{Overview of the main findings}

From the systematic review and the pooled analysis, developed in Chapter 5, genetic notification of smoking related disorders was suggested to improve smoking cessation in comparison to control at short-term follow-up, less than 6 months (relative risk ratio $=1.55,95 \%$ confidence interval 1.o9-2.21). A recent meta-analysis reported the same primary outcome [155]. However, differences were observed in the methods used. In our study, we decided to focus on randomised controlled trials for their ability to minimise the likelihood of systematic errors. Smerecnik et al. [155] included both randomised and non-randomised trials. In order to avoid overestimation of a single study, we considered one follow-up of each study in the pooled analysis (last follow-up or short-term smoking cessation), whereas this was not the case in the other study. We decided to use relative risk ratios (RR) rather than odds ratios (OR) for their easiness of interpretation and for their improved accuracy in prospective studies. In our study we also assessed secondary outcomes (intention-to-quit smoking, emotional outcome and recall and understanding of the genomic information). Despite these differences the main outcome of both studies is similar, which reinforces the validity of the results.

In Chapter 6, a review of posts discussing genetics and smoking was conducted on YouTube, Facebook and Twitter using two different time points (September 18, 2012 and May 7, 2013). Little information about genetics and smoking was reported in these three social media and even none in Facebook. However, this is expected to change in the near future due to the growing interest in genetic research in the general population. Google search was used as an indicator of the information available on the Internet and a source of other social media than YouTube, Facebook or Twitter. Websites retrieved through the Google search were more often scientific publications per se $(46.5 \%)$ or referring to scientific publications $(53.5 \%)$ than on YouTube or Twitter. Moreover, no social media such as blogs or Forum were identified in the Google search.

A survey in university students assessing the search of information about health, smoking and genetics was described in Chapter 7. Results indicated that Internet tools were mainly used to search for information about health and in much less case to search about smoking and genetics. Web-search engine and Forum were the two mostly used Internet tools followed by, respectively, YouTube, Facebook and Twitter. Moreover, factors explaining the search for information about health, smoking and genetics on Internet tools were gender, smoking status and the use of social media 
in univariate analyses and monthly budget, ethnicity, faculty, and smoking status in multivariate analyses.

\section{Limitations}

In Chapter 5, the main limitation of the pooled-analysis about the impact of genetic notification on smoking cessation was the low number of included studies inducing lower sensitivity in the test. Moreover, the outcome measures differed across studies (e.g. prolonged or point-prevalence smoking cessation) being one factor inducing heterogeneity between the included studies.

Social media, due to their easiness to create new information, are rapidly changing channels as reported in Chapter 6. Consequently, the posts selected in our review may not be reflective of what is posted at another time. Therefore, two different time points separated at 9 months in the time were used to collect information. Moreover, the search strategy using only two terms 'smoking' and 'genetic' and the first hundred posts may appear to be limited. However, the results obtained in our search provide a reasonable perspective of what someone interest in smoking and genetics would find on the topic on YouTube, Facebook and Twitter.

In Chapter 7, the sample of students having answered to the web-questionnaire might not be representative of the total population of university students. Indeed, 9 faculties were conveniently selected over the 14. Moreover, students only received one email inviting them to answer to the web-questionnaire. However, around 10\% of students answered to the questionnaire, which is comparable to other studies [309]. Furthermore, the cross-sectional design used in the study might have induced recall biases. Therefore, future prospective studies will be needed to confirm our results even if Internet tool were already reported to be used to search for information about health [298, 300, 302].

\section{Implications for clinical practice and future research}

Genetic notification mostly focused on one single variant in the literature. However, many variants are suggested to be associated with smoking initiation, addiction, cessation and smoking related disorders. Therefore, future study assessing the impact of genetic notification should include multiple variants. Genetic notification appeared to enhance smoking cessation only at short-term follow-up. It may suggest that reminders of the results might be necessary at longer term follow-up. In the future, genetic notification may also apply to epigenomics. By using epigenomic factors, the notification may evolve during time depending, for example, on the actual smoking status of the smoker. Indeed, methylations are found to be rapidly reversible and, therefore, remethylations are suggested after smoking cessation [31]. Consequently, impact on longer follow-up are plausible due to the constant and dynamic change in the test results over time. 
As reported in Chapter 7, students are often searching for information about health, smoking and genetics on Internet tools. Consequently, Internet tools might be used in public health to enhance the health and the genetic literacy of the population and thus to induce behaviour changes. As the population is more and more searching for information about health, smoking and genetics on the Internet and on social media, doctors and clinical researchers have to be part of the information displayed on the Internet. Moreover, they have to be ready to answer questions of the population based on what people might have read on the web.

As Internet tools and social media allow everybody to create new information, there is a need to monitor health information displayed over time to avoid the dispersion of false or misleading information and to manage the quantity of the information. Moreover, to enhance and develop faster translation of genome-based information from bench to bedside and even beyond to healthcare, methods may be developed. These methods might assess the type of Internet tools preferred to obtain health information (e. g. YouTube or Forum), the type of believed publisher (e.g. scientific or peers), and the preferred message content (e.g. paternalistic versus autonomy or positive-framed versus negative-framed messages). A web-questionnaire sent to one or different target populations (e. g. pregnant women or university students) may help in developing methods. Indeed, most likely the type of preferred Internet tools, the type of believed publisher and the preferred message content will differ from one target population to another one. Finally, the effectiveness of displaying such health messages on Internet tools might be assessed in real life. However, the control of people searching for such information remains challenging due to the anonymous access of information on the Internet.

\section{CONCLUSION}

Based on the results of the thesis, conclusions can be drawn with regard to the two main parts of the thesis: improving healthcare for smokers and enhancing the communication of genomic risks about smoking in the general population.

\section{Improving healthcare for smokers}

The use of genome-based information, in combination with a better overview of environmental factors influencing smoking, may improve the prevention of smoking initiation and addiction, as well as the treatment of smoking cessation. For example, in smoking cessation, genome-based information might be used to personalise and individualise interventions based on the choice of the drug, dose and duration. Improving healthcare for smokers is not only limited to research on smoking initiation, addiction and cessation but also on smoking related disorders like cancer or cardiovascular diseases as we observed genetic similarities between TUD and other disorders. 
Enhancing communication of genomic risks about smoking in the general population

The communication of genome-based information about smoking is of main importance to improve the health and the genomic literacy of the population. Multiple variants and epigenomic markers might be used to be closer to the reality and to enhance long-term impact of the notification. Both personalised (based on the genetic susceptibility of the individual) and generalised (based on the genetic risk associated to smoking or smoking related disorders) information to the population may be helpful. Therefore, developing reliable information through highly used media such as the Internet and social media is needed. Health information displayed on such media might be monitored and methods should be developed to increase the translation of scientific results for the general population. 



\section{INTRODUCTION}

Public Health Genomics (PHG) is the field of translating genome-based information and technologies into policies and healthcare. Among its many tasks, PHG provides also new insights in the prevention and the treatment of complex disorders and traits such as smoking. Despite the widespread knowledge of consequences of smoking, smoking still remains a major public health problem worldwide. Genomic factors and their interactions with environmental factors seem to be part of the causal mechanisms leading to smoking addiction and influencing smoking cessation. This is why, by improving the translation from bench to bedside and even beyond healthcare, PHG is a very innovative approach for tackling smoking in a most holistic way leading to targeted and personalised interventions.

Two broad groups of genes influence smoking initiation, addiction, cessation, and smoking related disorders. The first one includes genes influencing nicotine metabolism, such as $C Y P_{2} A 6$. Those genes are enzymes responsible for the metabolisation of nicotine into cotinine. The second group of genes consists of four different neurotransmitters (serotonin, mu-opioid, gamma-aminobutyric acid, and dopamine) influencing the reward.

The main objective of the present thesis is to demonstrate that the use of genomebased information, which goes beyond the influence of single or combined genes, is essential for further improvement in preventing and treating smoking. This thesis meets the challenge to use genome-based information arising from basic science in order to improve public health. The first aim of the present thesis was to provide research on how to improve healthcare for smokers (Chapter 2, Chapter 3 and Chapter 4). The second aim was to enhance the communication on the evidence of genomic risks about smoking to the population (Chapter 5 , Chapter 6 and Chapter 7).

\section{IMPROVING HEALTHCARE FOR SMOKERS}

Chapter 2 provides a literature review of the genetic predictors for smoking initiation and smoking cessation. Two main classes of genes influence smoking initiation and cessation (i) genes influencing the cascade theory of reward and (ii) genes influencing nicotine metabolism. Those genes were also reported to interact with intervention during smoking cessation. Moreover, 'omics' factors are also suggested to influence smoking initiation and cessation even if to date, few studies did such research. 
The environmental and genomic factors as well as interventions influencing smoking cessation were reviewed and visually displayed through the development of a working model described in Chapter 3. Smoking cessation was dynamically influenced by a broad range of factors and interventions. This includes genomic, smoking behaviour, demographic, socioeconomic status, health, psychological, biological, and social factors. Interventions were classified in three levels of target being individual, neighbourhood and society level.

The genetic similarities between disorders associated with tobacco use disorder are reported in Chapter 4. Based on a literature review and data-mining, 32 genes and 14 disorders met our inclusion criteria. Disorders included an extensive range of categories such as addictions, mental disorders, cardiovascular disorders, and cancer. Genetic similarities were observed between two main clusters of disorders. The first one included mostly disorders that are side effects of smoking (e. g. vascular disorder and oesophageal cancer). The second one targeted coexisting disorders of smoking (e.g. alcoholism and depressive disorder).

ENHANCING THE COMMUNICATION OF GENOMIC RISKS ABOUT SMOKING IN THE GENERAL POPULATION

Chapter 5 provides a systematic review and a meta-analysis describing the impact of genetic notification on smoking cessation. Eight papers, assessing the impact of cancer genetic risk communication, were included in the review. In short-term follow-up (less than 6 months), the pooled-analysis suggested an improvement of smoking cessation in people receiving genetic notification in comparison to controls (relative risk ratio $=1.55,95 \%$ confidence interval 1.09-2.21).

Chapter 6 describes the availability of genetic information about smoking in three different social media (YouTube, Facebook, and Twitter). Over 31, o and 84 posts were retrieved from, respectively, YouTube, Facebook and Twitter. Most posts referred to scientific publications and focused on smoking addiction and smoking related disorders. Improving access to such information might improve the health and the genomic literacy of the population and, therefore enhance smoking prevention and cessation.

Chapter 7 reports the Internet tools used and the factors explaining the search of information about health, smoking and genetics on the Internet. Out of the 2,171 students that answered the full questionnaire, 1,937 searched for information about at least health, smoking or genetics on Internet tools. The two mostly used Internet tools were web-search engine and Forum. The other factors explaining the search of information about health, smoking or genetics were gender, student's housing, smoking status and the use of social media in univariate analyses; and monthly budget, nationality and the faculty of study in multivariate analyses. Due to the important search of such information on Internet tools, further research should assess and monitor the quality of the information displayed. 
Chapter 8 summarises the main findings and the implications for public health practice and future research of the present thesis for the two main parts of the study (i) improving healthcare for smokers and (ii) enhancing the communication of genomic risks about smoking in the general population. Results of our research indicated an important need (a) to include both environmental and genomic factors associated to smoking, (b) to better understand the biological mechanisms leading to smoking and smoking related disorders to improve healthcare for smokers by targeted interventions, and (c) to develop methods and communication strategies that may enhance the translation of genome-based information on smoking to the general population. Two different types of communication were developed, the use of genetic notification to boost smoking cessation and the use of Internet tools and social media to improve the genomic literacy of the population. Genetic notification is, to date, mainly studied with one single gene variant. In the future, multiple genetic variants and most importantly epigenomic markers and exposomics might be used to be closer to the reality and to enhance long-term impact of the notification. The Internet and social media, as tools for information displaying, sharing and gathering, will become an increasingly important place to improve the health and the genomic literacy of the population. Therefore, there is a need to monitor the displayed health information and to develop methods that may increase the translation of results from basic science to the general population. 


\section{INTRODUCTIE}

'Public Health Genomics' (PHG) is de manier om genomische informatie en technologieën in het beleid en de gezondheidszorg te vertalen. Onder haar vele taken biedt PHG ook nieuwe inzichten in de preventie en behandeling van complexe aandoeningen en verslavingen zoals roken. Ondanks de wijdverspreide kennis van de gevolgen van roken blijft roken een belangrijk probleem voor de wereldwijde volksgezondheid. Genomische factoren en hun interacties met omgevingsfactoren lijken een deel te zijn van de causale mechanismen die tot een rookverslaving leiden en die de efficiëntie van het stoppen met roken beïnvloeden. Dit is de reden waarom - met een verbeterde toepassing van praktische en theoretische concepten bij patiënten en zelfs buiten gezondheidszorg - PHG een zeer innovatieve benadering is voor de aanpak van roken op een zeer holistische wijze die tot gerichte en gepersonaliseerde interventies kan leiden.

Twee grote groepen van genen beïnvloeden het starten met roken, de verslaving, het stoppen en aan roken gerelateerde aandoeningen. De eerste groep bevat genen die het metabolisme van de nicotine beïnvloeden zoals $C Y P_{2} A 6$. Deze genen zijn enzymen die verantwoordelijk zijn voor het metabolisme van de nicotine in cotinine. De tweede groep genen bestaat uit vier verschillende neurotransmitters (serotonine, mu-opioid, gamma-aminoboterzuur en dopamine) die de 'beloning' van het roken beïnvloeden.

Deze thesis stelt tot doel de op de genoom-gebaseerde informatie uit de fundamentele wetenschap te gebruiken ter verbetering van de volksgezondheid. De eerste doelstelling van dit proefschrift was het genomische onderzoek te gebruiken om te bezien hoe de gezondheidszorg voor rokers verbeterd kan worden (Hoofdstuk 2, Hoofdstuk 3 en Hoofdstuk 4). De tweede doelstelling was de communicatie met de bevolking over het bewijs van genomische risico's bij roken te verbeteren (Hoofdstuk 5, Hoofdstuk 6 en Hoofdstuk 7).

IMPROVING HEALTHCARE FOR SMOKERS

Hoofdstuk 2 betreft een literatuuroverzicht van de genetische voorspellers die een rol bij het starten en stoppen met roken spelen. Twee belangrijke klassen van genen beïnvloeden het starten en stoppen met roken: (i) genen die de cascade van de beloning beïnvloeden en (ii) genen die betrokken zijn bij het nicotinemetabolisme. Deze genen werden ook gerapporteerd als betrokken zijnde bij de interacties die het stoppen met roken beïnvloeden. Bovendien worden 'omics'-factoren er ook van 
verdacht het starten en het stoppen met roken te beïnvloeden, hoewel tot nog toe weinig studies een dergelijk onderzoek uitvoerden.

De milieu- en genomische factoren evenals de interventies die het stoppen met roken beïnvloeden werden beoordeeld en visueel weergegeven in een werkmodel. Dit wordt in Hoofdstuk 3 beschreven. Stoppen met roken is via een breed scala aan factoren en interventies dynamisch te beïnvloeden. Deze omvatten genomica, rookgedrag, demografie, sociaaleconomische status, gezondheid en psychologische-, biologische - en sociale factoren. De interventies werden in drie doelgroepen ingedeeld: het individuele-, het buurt- en het maatschappelijke niveau.

De genetische overeenkomsten tussen de verschillende aandoeningen die met tabaksmisbruik worden geassocieerd worden in Hoofdstuk 4 beschreven. Op basis van een literatuurstudie en data-extractie voldeden 32 genen en 14 aandoeningen aan onze inclusiecriteria. De betreffende aandoeningen omvatten een breed scala aan categorieën zoals verslaving, psychische stoornissen, hart- en vaatziekten en kanker. Genetische overeenkomsten werden bij twee belangrijke clusters van aandoeningen gevonden. De eerste cluster betrof voornamelijk aandoeningen die een bijwerking van roken zijn (bv. vasculaire aandoeningen en slokdarmkanker). De tweede cluster was op de coëxisterende aandoeningen van roken gericht (bv. alcoholisme en depressieve stoornis).

VERBETERING VAN DE COMMUNICATIE OVER GENOMISCHE RISICO'S VAN ROKEN IN DE ALGEMENE BEVOLKING

Hoofdstuk 5 betreft een systematisch overzicht en een meta-analyse die de invloed van genetische kennisgeving op het stoppen met roken beschrijven. In het overzicht werden acht artikelen over de impact van communicatie over het genetische risico op kanker opgenomen. In een korte termijn follow-up (minder dan zes maanden) constateerde de pool-analyse een verbeterd resultaat ten aanzien van het stoppen met roken bij de mensen met een genetische kennisgeving in vergelijking tot de controlegroep (relatieve risico ratio $=1,55,95 \%$ betrouwbaarheidsinterval 1,09-2,21).

Hoofdstuk 6 beschrijft de beschikbaarheid van genetische informatie over roken in drie verschillende sociale media (YouTube, Facebook en Twitter). Bovendien 31, o en 84 berichten werden respectievelijk uit YouTube, Facebook en Twitter opgehaald. De meeste berichten refereerden aan wetenschappelijke publicaties en waren op rookverslaving en aan roken gerelateerde aandoeningen gericht. Een verbeterde toegankelijkheid tot dergelijke informatie kan de gezondheid en de 'genomische' geletterdheid van de bevolking verbeteren. Dit kan rookpreventie bevorderen en de resultaten bij het stoppen met roken verbeteren.

Hoofdstuk 7 beschrijft de middelen die via het Internet gebruikt zijn en de verklarende factoren voor het zoeken van informatie over gezondheid, roken en genetica op het Internet. Van de 2.171 studenten die de volledige vragenlijst beantwoord hebben zochten ten minste 1.937 studenten naar informatie over gezondheid, roken of genetica op het Internet. De twee meest gebruikte Internet tools waren 
web-zoekmachines en Fora. De andere factoren die het zoeken naar informatie over gezondheid, roken of genetica verklaarden waren het geslacht, de huisvesting van de student, de rookstatus en het gebruik van sociale media in de univariate analyses en het maandelijkse budget, de nationaliteit en de studiefaculteit in de multivariate analyses. Vanwege het belang van de zoektocht naar dergelijke informatie op Internet zou verder onderzoek deze informatie moeten beoordelen en de kwaliteit van de getoonde informatie moeten bewaken.

\section{CONCLUSIONS AND PERSPECTIVES}

Hoofdstuk 8 is een samenvatting van de belangrijkste bevindingen, de implicaties voor de volksgezondheidspraktijk en het gewenste vervolgonderzoek voor de twee belangrijkste onderdelen van het onderzoek: (i) de verbetering van de gezondheidszorg voor rokers en (ii) het verbeteren van de communicatie over genomische risico's van roken in de algemene populatie. De resultaten van ons onderzoek wezen op een belangrijke behoefte (a) om zowel milieu- als genomische factoren die met roken verband houden te betrekken, (b) een beter inzicht te hebben in de biologische mechanismen die leiden tot roken en aan roken gerelateerde aandoeningen om de gezondheidszorg voor rokers door gerichte interventies te verbeteren en (c) om methoden en communicatiestrategieën te ontwikkelen die de genoom-gebaseerde informatie over roken naar de algemene bevolking kunnen vertalen. Twee verschillende vormen van communicatie werden ontwikkeld; het gebruik van genomische kennisgeving bij het stoppen met roken en het gebruik van Internet tools en sociale media om de genomische geletterdheid van de bevolking stimuleren. Genetische kennisgeving is tot op heden vooral bij een enkel gen variant bestudeerd. In de toekomst zouden verschillende genetische varianten alsook epigenomische markers en exposomics kunnen worden bestudeerd om een completer beeld te krijgen en om de langetermijneffecten van roken op een zo efficiënt mogelijk manier te verminderen. Het Internet en de sociale media als instrumenten voor het weergeven, delen en verzamelen van informatie zullen steeds belangrijker worden om de gezondheid en de genomische geletterdheid van de bevolking te verbeteren. Daarom bestaat de behoefte om de weergegeven informatie over gezondheid te monitoren en methoden te ontwikkelen die resulteren in een heldere en begrijpelijke vertaling van fundamentele wetenschappelijke resultaten naar de algemene bevolking 
RÉSUMÉ

\section{INTRODUCTION}

La Santé Publique Génomique (PHG) a pour objectif de traduire les informations et technologies basées sur la génomique en pratique clinique et politiques de santé. Parmi ses multiples tâches, la PHG propose un nouveau regard sur la prévention et le traitement de maladies complexes telles que l'addiction au tabac. Malgré l'importante connaissance des conséquences du tabac sur la santé, l'addiction au tabac reste un problème majeur de santé publique. Les facteurs génomiques ainsi que les interactions avec les facteurs environnementaux semblent faire partie du mécanisme causal menant à l'addiction au tabac et influençant l'arrêt du tabac. C'est pourquoi, en améliorant la traduction de la recherche, du laboratoire au chevet du patient et même au-delà de la pratique clinique, la PHG aborde le problème de la consommation de tabac de manière innovative et holistique menant à des interventions plus ciblées et personnalisées.

Deux grands groupes de gènes influencent l'initiation, l'addiction, l'arrêt du tabac ainsi que les maladies liées au tabac. Le premier groupe inclut les gènes influençant le métabolisme de la nicotine tels que $C Y P_{2} A 6$. Ces gènes sont des enzymes responsables de la métabolisation de la nicotine en cotinine. Le second groupe de gènes contient quatre neurotransmitteurs (la sérotinine, les mu-opioid, les acides gamma-aminobutyriques et la dopamine) influençant le sentiment de récompense.

L'objectif principal de cette thèse est de démontrer que l'utilisation d'informations basées sur la génomique, allant au-delà de l'influence d'un seul ou d'une combinaison de gènes, est essentiel afin d'améliorer la prévention ainsi que l'arrêt du tabac. Cette thèse rencontre donc un défi, celui d'utiliser les informations basées sur la génomique résultant de la recherche scientifique de base afin d'améliorer la santé publique. Le premier objectif de cette thèse est, dans ce cadre, d'améliorer les soins de santé des fumeur (Chapitre 2, Chapitre 3 et Chapitre 4). Le second objectif est d'accroître la communication à propos de l'évidence des risques génomiques concernant le tabac (Chapitre 5, Chapitre 6 et Chapitre 7).

\section{AMÉLIORER LES SOINS DE SANTÉ DES FUMEURS}

Le Chapitre 2 décrit, sur base d'une revue de la littérature, les prédicteurs génétiques de l'initiation et l'arrêt du tabac. Deux classes de gènes influencent principalement l'initiation et l'arrêt du tabac (i) les gènes influençant le sentiment de récompense et (ii) les gènes influençant le métabolisme de la nicotine. Ces gènes ont aussi présenté des interactions avec différentes interventions lors de l'arrêt du tabac. De plus, une influence des facteurs '-omique' est aussi suggérée lors de l'initiation 
et l'arrêt du tabac même si, actuellement, peu d'études se sont penchées sur la question.

Les facteurs génomiques et environnementaux ainsi que les interventions influençant l'arrêt du tabac ont été examinés via une revue systématique de la littérature et présentés visuellement à l'aide d'un modèle de travail décrit au Chapitre 3 . L'arrêt du tabac est dynamiquement influencé par une large gamme de facteurs et d'interventions. Ceci inclut les facteurs génomiques, démographiques, socio-économiques, psychologiques, biologiques, sociaux et de santé ainsi que les facteurs déterminant le comportement tabagique. Les interventions ont été classifiées en trois niveaux de cibles: le niveau individuel, du voisinage et de la société.

Les similarités génétiques entre les maladies associées à l'utilisation du tabac sont rapportées au Chapitre 4. Sur base d'une revue de la littérature et de datamining, 32 gènes et 14 maladies ont recontré nos critères d'inclusion. Les maladies se rapportent à une gamme étendue de catégories telles que les addictions, les maladies mentales, les maladies cardiovasculaires et le cancer. Principalement deux clusters de maladies présentent des similarités génétiques. Le premier inclut des maladies se présentant comme des effets secondaires de la consommation de tabac (p. ex. les maladies vasculaires et le cancer de l'oesophage). Le deuxième cible des maladies coexistantes au tabac (p. ex. l'alcoolisme et les maladies dépressives).

ACCROÂTRE LA COMMUNICATION À PROPOS DE L'ÉVIDENCE DES RISQUES GÉNOMIQUES CONCERNANT LE TABAC DANS LA POPULATION GÉNÉRALE

Le Chapitre 5 présente une revue systématique de la littérature et une méta-analyse décrivant l'impact de la notification génétique sur l'arrêt du tabac. Huit publications évaluant l'impact de la communication de risques génétiques ont été inclues dans notre revue. La méta-analyse suggère une amélioration de l'arrêt du tabac à court terme (moins de 6 mois) chez les personnes recevant une information génétique en comparaison des contrôles (risque relatif $=1.55$, Intervalle de confiance à $95 \%$ 1.09-2.21).

Le Chapitre 6 décrit les informations disponibles à propos de la génétiques et du tabac dans trois média sociaux (YouTube, Facebook et Twitter). YouTube, Facebook et Twitter ont permis de sélectionner, respectivement, 31, o and 84 postes. La plupart des postes font référence à des publications scientifiques et se concentrent sur l'addiction au tabac et les maladies liées au tabac. Améliorer l'accès à de telles informations devrait permettre d'améliorer la santé ainsi que la connaissance en génomique de la population et, par conséquent, accroître la prévention ainsi que l'arrêt du tabac.

Le Chapitre 7 présente les outils Internet et les facteurs expliquant la recherche d'informations sur la santé, le tabac et la génétique sur Internet. Parmi les 2.171 étudiants qui ont répondu entièrement au questionnaire, 1.937 ont, par le passé, cherché de l'information sur la santé, le tabac ou sur la génétique via des outils Internet. Les deux outils Internet les plus utilisés sont les moteurs de recherche et 
les Forum. Les autres facteurs expliquant la recherche d'information sur la santé, le tabac ou la génétique sont le sexe, le type d'habitation, le statut tabagique et l'utilisation des média sociaux en analyses univariées; ainsi que le budget mensuel, la nationalité ainsi que la faculté d'étude en analyses multivariées. Compte tenue de l'importance de la recherche d'informations sur les outils Internet, davantage de recherches devraient évaluer et monitorer la qualité des informations disponibles.

\section{CONCLUSIONS ET PERSPECTIVES}

Le Chapitre 8 résume les principaux résultats de cette thèse ainsi que les implications pour la pratique en santé publique et les futures recherches concernant les deux parties principales de cette étude (i) améliorer les soins de santé des fumeurs et (ii) accroître la communication à propos de l'évidence des risques génomiques concernant le tabac dans la population générale. Les résultats de notre recherche indiquent un important besoin (a) d'inclure à la fois les facteurs environnementaux et les facteurs génomiques associés à la consommation de tabac, (b) d'améliorer la compréhension des mécanismes biologiques menant à la consommation de tabac et aux maladies liées au tabac afin d'améliorer les soins de santé des fumeurs à l'aide d'interventions ciblées et (c) de développer des méthodes ainsi que des stratégies de communication afin d'accroître la traduction des informations génomiques à propos du tabac dans la population générale. Deux différents types de communications ont été développés dans cette thèse, l'utilisation de notification génétique afin d'améliorer l'arrêt du tabac et l'utilisation d'outils Internet et de média sociaux pour accroître la connaissance en génomique de la population. La notification génétique est, actuellement, principalement étudiée à l'aide d'un seul variant génétique. Dans le futur, de multiples variants génétiques ainsi que des marqueurs épigénomiques et exposomiques devraient être utilisés afin d'être au plus proche de la réalité et donc d'améliorer l'impact à long terme de la notification. Internet et les media sociaux, en tant qu'outils d'information permettant de développer, partager et rassembler de l'information, représentent un espace important afin d'améliorer la santé et les connaissances en génétique de la population. Par conséquent, il y a un besoin de monitorer les informations de santé disponibles et de développer des méthodes permettant d'augmenter la traduction des résultats de la recherche scientifique de base pour la population générale. 



\section{ACKNOWLEDGEMENTS}

First, I want to thank the Scientific Institute of Public Health (WIV-ISP), and more specifically Prof. Dr. Herman Van Oyen and Dr. Johan Van der Heyden, who offered me the possibility to do this PhD thesis on Public Health Genomics.

Thank you Herman for your guidance, your openness in all my projects and your constructive advices. Johan, it was a real pleasure to work with you. Thank you for your continued support and your advices at times of critical need. Marc, thank you for our different discussions about genomics!

I also want to thank different colleagues without whom I would probably not have achieved this thesis. A special thanks to Rana, your experience as former PhD candidate was really important to me. Helene, thanks for our discussions on how to change the world. Thanks to many other colleagues who were on my road during those three last years.

Then, I want to thank Maastricht University, the Institute for Public Health Genomics (IPHG) and more specifically Prof. Dr. Angela Brand and Prof. Dr. Servaas Morré for being my host University.

Angela, thank you for the autonomy given during this thesis and your confidence in my work. Servaas, thanks for your support during these three years and your help in all my daily life questions, problems, ... ! Now that I am finished with this $\mathrm{PhD}$, I am thinking to develop research on Coke addiction. Shall we use Pepsi addiction for the control group?

Thanks to all of you within the IPHG for making my days in Maastricht funny, scientifically, and culturally interesting. A special thanks to Yvonne who took care of all the administrative points such as scheduling my meetings. You really made my stays in Maastricht more 'cost-efficient'. And another special thanks to my two paranymphs, Tomris and Ivan.

I also want to thank all my co-authors for the nice team working and their help in making my publications possible.

Less scientific but almost equally important to achieve the end of this thesis: A special thanks to all the singers that made me concentrated when I was working. Thanks to Béa, the FASC, the GFB, ... who made my leisure time SUPERproductive! Thanks to my parents and family for their presence.

Finally, I want to thank YOU for being there and supporting me during this last stage of my PhD! 
Sylviane de Viron was born in Brussels, Belgium, on the $11^{\text {th }}$ of July 1986. In 2004, she acquired her secondary school diploma at the Institut Saint André (Brussels). In September of the same year, she started a bachelor in Nursing followed by a specialization in Paediatrics in Brussels. In September 2008, she started a master of Public Health at the Catholic University of Louvain (UCL), Brussels. As part of this master, she followed several elective courses and more particularly a specialization on genetic epidemiolgy at the Ludwig Maximilians University (LMU), Munich. During her master, she assessed the genetic and clinical determinants of pulse wave velocity in renal transplant patients. She obtained her degree in September 2010. After her master, she worked as teaching and research assistant at the public health faculty of the UCL.

In January 2011, Sylviane started working as scientific researcher within the Scientific Institute of Public Health (WIV-ISP), Brussels, and as PhD candidate within the Institute for Public Health Genomics (IPHG) at Maastricht University on the project described in this dissertation. The project was granted by the Belgian Federal Science Policy Office. During her PhD, she presented orally the results of her research to different national and international congresses such as the World Cancer Congress 2012 and the European Public Health Conference 2012. She also took part in the group of Public Health Genomics within the Belgian Health Council and the Public Health Genomics European Network coordinated by Prof. Dr. Angela Brand. 
de Viron S, Van der Heyden J, Ambrosino E, Arbyn M, Brand A, Van Oyen H: Impact of genetic notification on smoking cessation: Systematic review and pooledanalysis. Plos One. 2012. 7: e40230. DOI: 10.1371/journal.pone.0040230.

de Viron S: Genetic predictors for smoking initiation and cessation. In Advances in smoking cessation. Edited by Polosa R, Caponnetto P. London, UK: Future Medicine. 2013. 98-114. DOI: 10.2217/EBO.12.233.

de Viron S, Malats N, Van der Heyden J, Van Oyen H, Brand A: Environmental and Genomic Factors as well as Interventions Influencing Smoking Cessation: A Systematic Review of Reviews and A Proposed Working Model. Public Health Genomics. 2013. 16(4): 159-173. DOI: 10.1159/000351453.

de Viron S, Suggs LS, Brand A, Van Oyen H: Communicating genetics and smoking through social media: Are we there yet? J. Med. Internet Res. 2013. 15(9):e198. DOI: 10.2196/jmir.2653.

de Viron S, Morré SA, Van Oyen H, Brand A, Ouburg S: Genetic similarities between comorbidities of tobacco use disorders: an exploratory study. [Submitted].

de Viron S, Brand A, Morré SA, Czabanowska K, Van Oyen H: Which Internet tools are searched by students at University to obtain information about health, smoking and genetics? A pilot study. [Submitted].

\section{ADDITIONAL PUBLICATIONS AND REPORTS}

de Viron S, Van der Heyden J, Ambrosino E, Arbyn M, Brand A, Van Oyen H: Impact of genetic notification on smoking cessation: Pooled analysis. In WIV-ISP scientific report 2010-2011. Edited by Peeters J. Brussels. 2012:45-47.

de Viron S, Van Oyen H: How Public Health Genomics could enhance smoking cessation? The Newsletter of the British Society for Human Genetics. 2013, 58. 



\section{REFERENCES}

[1] Genome-based research and population Health.Report of an expert workshop held at the rockefeller foundation study and conference centre bellagio, italy, 14-20 april. Technical report, 2005.

[2] W. Burke, M.J. Khoury, A. Stewart, and R.L. Zimmern. The path from genome-based research to population health: development of an international public health genomics network. Genet.Med., 8(1098-3600 (Print)):451458 , July 2006.

[3] M.J. Khoury, M. Gwinn, P.W. Yoon, N. Dowling, C.A. Moore, and L. Bradley. The continuum of translation research in genomic medicine: how can we accelerate the appropriate integration of human genome discoveries into health care and disease prevention? Genet.Med., 9(1530-0366 (Electronic)):665-674, October 2007.

[4] A. Brand and J.A. Lal. European best practice guidelines for quality assurance, provision and use of genomebased information and technologies: the 2012 declaration of rome. Drug Metabol.Drug Interact., 27:177-182, 2012.

[5] A. Jemal, M.J. Thun, L.A. Ries, H.L. Howe, H.K. Weir, M.M. Center, E. Ward, X.C. Wu, C. Eheman, R. Anderson, Ajani U.A., B. Kohler, and Edwards B.K. Annual report to the nation on the status of cancer, 1975-2005, featuring trends in lung cancer, tobacco use, and tobacco control. J.Natl Cancer Inst., 100:1672-1694, 2008.

[6] E. Cleeren, J. Van der Heyden, A. Brand, and H. Van Oyen. Public health in the genomic era: will public health genomics contribute to major changes in the prevention of common diseases? Archives of Public Health, 69(1), 2011.

[7] R. Zimmern and A. Stewart. Public health genomics: origins and basic concepts. IJPH, 3(3-4):9-15, 2006.

[8] A. Brand. Public health and genetics-a dangerous combination? Eur.J.Public Health, 15(1101-1262 (Print)):114-116, April 2005.

[9] G.D. Smith, S. Ebrahim, S. Lewis, A.L. Hansell, L.J. Palmer, and P.R. Burton. Genetic epidemiology and public health: hope, hype, and future prospects. Lancet, 366(1474-547X (Electronic)):1484-1498, October 2005.

[10] L.M. Beskow, M.J. Khoury, T.G. Baker, and J.F. Thrasher. The integration of genomics into public health research, policy and practice in the united states. Community Genet., 4(1422-2833 (Electronic)):2-11, July 2001.

[11] T.A. Pearson and T.A. Manolio. How to interpret a genome-wide association study. Journal of the American Medical Association, 299(1538-3598 (Electronic)):1335-1344, March 2008.

[12] M.J. Khoury and G.A. Mensah. Genomics and the prevention and control of common chronic disease emerging priorities for public health action. Prev Chronic Dis, 2(2):A05, April 2005.

[13] M.J. Khoury and J.S. Dorman. The human genome epidemiology network. American Journal of Epidemiology, 148 (1):4, July 1998.

[14] Asthma genomics: implications for public health. Technical report, University of Washington, Center of genomics and public health, 2004 .

[15] G.D. Smith, M. Gwinn, S. Ebrahim, L.J. Palmer, and M.J. Khoury. Make it HuGE: human genome epidemiology reviews, population health, and the IJE. Int.J.Epidemiol., 35(0300-5771 (Print)):507-510, June 2006.

[16] J.A. Lal, T. Schulte In den Baumen, S.A. Morre, and A. Brand. Public health and valorization of genome-based technologies: a new model. J.Trasl.Med, 9:207, 2011.

[17] A. Brand. Public health genomics and personalized healthcare: a pipeline from cell to society. Drug Metab. Drug Interact., 27:121-123, 2012.

[18] Institude of Medicine (IOM). Who will keep the public healthy? educating public health professionals for the 21st century. Technical report, Institute of Medicine (IOM), Bethesda (MD), 2003.

[19] M. Eriksen, J. Mackay, and H. Ross. The tobacco atlas: Fourth ed., 2012. 
[20] H.H. Lin, M. Ezzati, and M. Murray. Tobacco smoke, indoor air pollution and tuberculosis: a systematic review and meta-analysis. PloS Med., 4:e2o, 2007.

[21] S. Neubauer, R. Welte, A. Beiche, H.H. Koenig, K. Buesh, and R. Leidl. Mortality, morbidity and costs attributable to smoking in germany: update and a 10-year comparison. Tob. Control, 15:464-471, 2006.

[22] World Health Organisation. The world health report 2002. Technical report, Geneva, 2002.

[23] M.D. Li, R. Cheng, J.Z. Ma, and G.E. Swan. A meta-analysis of estimated genetic and environmental effects on smoking behavior in male and female adult twins. Addiction, 98(0965-2140 (Print)):23-31, January 2003.

[24] J.M. Vink, G. Willemsen, and D.I. Boomsma. Heritability of smoking initiation and nicotine dependence. Behav.Genet., 35(Ooo1-8244 (Print)):397-406, July 2005.

[25] H. Xian, J.F. Scherrer, P.A. Madden, M.J. Lyons, M. Tsuang, W.R. True, and S.A. Eisen. The heritability of failed smoking cessation and nicotine withdrawal in twins who smoked and attempted to quit. Nicotine.Tob.Res., 5 (1462-2203 (Print)):245-254, April 2003.

[26] K. Blum, E.R. Braverman, J.M. Holder, J.F. Lubar, V.J. Monastra, D. Miller, J.O. Lubar, T.J. Chen, and D.E. Comings. Reward deficiency syndrome: a biogenetic model for the diagnosis and treatment of impulsive, addictive, and compulsive behaviors. J.Psychoactive Drugs, 32 Suppl(o279-1072 (Print)):i-112, November 2000.

[27] M. Munafo, T. Clark, E. Johnstone, M. Murphy, and R. Walton. The genetic basis for smoking behavior: a systematic review and meta-analysis. Nicotine.Tob.Res., 6(1462-2203 (Print)):583-597, August 2004.

[28] Mechanistic links between copd and lung cancer. Nature Reviews Cancer, author $=$ Houghton, A., year $=2013$, pages $=233-245,13$.

[29] K. Wanke and E. Augustson. Epidemiological analysis of variation in phenotypic definitions: a proof of concept using an example of a cessation phenotype, pages 339-402. NCI Tobacco Control Monographs. U.S. Department of Health and Human Services, National Institutes of Health, National Cancer Institute, Bethesda, MD, August 2009.

[30] J.S. Brody. Transcriptome alterations induced by cigarette smoke. Int.J.Cancer, 131(12):2754-2762, 2012.

[31] E.S. Wan, W. Qiu, A. Baccarelli, V.J. Carey, H. Bacherman, S.I. Rennard, A. Agusti, W. Anderson, D.A. Lomas, and D.L. Demeo. Cigarette smoking behaviors and time since quitting are associated with differential DNA methylation across the human genome. Hum.Mol.Genet., 21(13), April 2012.

[32] W.H. Berrettini, L. Bierut, T.J. Crowleyr, J.F. Cubells, J. Frascella, J. Gelernter, J.K. Hewitt, M.J. Kreek, Lachman H., M. Leppert, M.D. Li, P. Madden, C. Miner, J.D. Pollock, O. Pomerleau, J.P. Rice, J.L. Rutter, D. Shurtleff, G.E. Swan, J.A. Tischfield, M. Tsuang, G.R. Uhl, M. Vanyukov, N.D. Volkow, and K. Wanke. Setting priorities for genomics research. Science, 304:1445-1446, 2004.

[33] C.M. McBride, D. Bowen, L.C. Brody, C.M. Condit, R.T. Croyle, M. Gwinn, M.J. Khoury, L.M. Koehly, B.R. Korf, T.M. Marteau, K. McLeroy, K. Patrick, and T.W. Valente. Future health applications of genomics: priorities for communication, behavioral, and social sciences research. Am.J.Prev.Med., 38(1873-2607 (Electronic)):556-565, May 2010.

[34] A.D. Bryan and K.E. Hutchison. The role of genomics in health behavior change: challenges and opportunities. Public Health Genomics, 15:139-145, 2012.

[35] M.J. Dingel, A.D. Hicks, M.E. Robinson, and B.A. Koenig. Integrating genetic studies of nicotine addiction into public health practice: stakeholder views on challenges, barriers and opportunities. Public Health Genomics, 15: 46-55, 2012.

[36] M. Gwinn, M. Bowen, and M. Khoury. Genomics and public health at CDC. MMWR Morb.Mortal.Wkly.Rep., 55 Suppl 2(1545-861X (Electronic)):20-21, December 2006.

[37] J.E. Rose, F.M. Behm, T. Drgon, C. Johnson, and G.R. Uhl. Personalized smoking cessation: interactions between nicotine dose, dependence and quit-success genotype score. Mol.Med., 16(1528-3658 (Electronic)):247-253, July 2010.

[38] H. Li, L. Yangn, X. Zhao, J. Wang, J. Qian, H. Chen, W. Fan, H. Liu, L. Jin, W. Wang, and D. Lu. Prediction of lung cancer risk in a chinese population using a multifactorial genetic model. BMC Med. Genet., 13:118, 2012.

[39] B.W. Hesse, N.K. Arora, and M.J. Khoury. Implications of internet availability of genomic information for public health practice. Public Health Genomics, 15:201-208, 2012. 
[40] B.B. McGrath. Advancing the post-genomic era agenda: contributions from public health. Public Health Genomics, 15:125-131, 2012.

[41] I. Ishiyama, A. Nagai, K. Muto, A. Tamakoshi, M. Kokado, K. Mimura, T. Tanzawa, and Z. Yamagata. Relationship between public attitudes toward genomic studies related to medicine and their level of genomic literacy in japan. American Journal of Medical Genetics Part A, 146:1696-1706, 2008.

[42] L. Wagner, R. Paquin, and S. Persky. Genetics blogs as a public health tool: assessing credibility and influence. Public Health Genomics, 15:218-225, 2012.

[43] N. Hamajima, Y. Atsuta, Y. Goto, and H. Ito. A pilot study on genotype announcement to induce smoking cessation by japanese smokers. Asian Pacific journal of cancer prevention : APJCP, 5(1513-7368 (Print)):409-413, October 2004.

[44] N. Hamajima, K. Suzuki, Y. Ito, and T. Kondo. Genotype announcement to japanese smokers who attended a health checkup examination. J.Epidemiol., 16(0917-5040 (Print)):45-47, January 2006.

[45] A. Hishida, T. Terazawa, T. Mamiya, H. Ito, K. Matsuo, K. Tajima, and N. Hamajima. Efficacy of genotype notification to japanese smokers on smoking cessation-an intervention study at workplace. Cancer Epidemiology, 34(1):96-100, 2010. ISSN 1877-7821.

[46] H. Ito, K. Matsuo, K. Wakai, T. Saito, H. Kumimoto, K. Okuma, K. Tajima, and N. Hamajima. An intervention study of smoking cessation with feedback on genetic cancer susceptibility in japan. Prev.Med., 42(oog1-7435 (Print)):102-108, February 2006.

[47] M. Kano, Y. Goto, Y. Atsuta, M. Naito, and N. Hamajima. Smoking cessation after genotype notification: pilot studies of smokers employed by a municipal government and those on nagoya university medical campus. Nagoya journal of medical science, 69(0027-7622 (Print)):149-156, October 2007.

[48] C.M. McBride, G. Bepler, I.M. Lipkus, P. Lyna, G. Samsa, J. Albright, S. Datta, and B.K. Rimer. Incorporating genetic susceptibility feedback into a smoking cessation program for african-american smokers with low income. Cancer Epidemiology Biomarkers and Prevention, 11(1055-9965 (Print)):521-528, June 2002.

[49] C.M. McBride, L.M. Koehly, S.C. Sanderson, and K.A. Kaphingst. The behavioral response to personalized genetic information: will genetic risk profiles motivate individuals and families to choose more healthful behaviors? Annual Review of Public Health, 31:89-103, 2010.

[50] A.J. Wright, D.P. French, J. Weinman, and T.M. Marteau. Can genetic risk information enhance motivation for smoking cessation? an analogue study. Health Psychol., 25(0278-6133 (Print)):740-752, November 2006.

[51] A.J. Wright, C. Takeichi, S.C. Whitwell, M. Hankins, and T.M. Marteau. The impact of genetic testing for crohn's disease, risk magnitude and graphical format on motivation to stop smoking: an experimental analogue study. Clin.Genet., 73(1399-0004 (Electronic)):306-314, April 2008.

[52] A.J. Wright, J. Weinman, and T.M. Marteau. The impact of learning of a genetic predisposition to nicotine dependence: an analogue study. Tob.Control, 12(1468-3318 (Electronic)):227-230, June 2003.

[53] R.L. Zimmern and M.J. Khoury. The impact of genomics on public health practice: the case for change. Public Health Genomics, 15:118-124, 2012.

[54] A.A. Vinkhuyzen, van der Sluis S., and Posthuma D. Behavioral genetics. In Carlstedt R.A., editor, Handbook of integrative clinical Psychology, Psychiatry and Behavioral medicine: Perspectives, Practices, and Research. Cambridge university press, 2010.

[55] L.J. Palmer, P.R. Burton, and G.D. Smith. An introduction to genetic epidemiology. The Policy Press Unversity of Bristol, 2011.

[56] F. Caraci and F. Drago. Nicotine addictions: neurobiological circuits and pharmacological targets, pages 24-37. Future Medicine, London, UK, 2013.

[57] Race Ethnicity and Genetics Working Group. The use of racial, ethnic, and ancestral categories in human genetics research. Am.J.Hum.Genet., 77(0002-9297 (Print)):519-532, October 2005.

[58] R. Ray, R.F. Tyndale, and C. Lerman. Nicotine dependence pharmacogenetics: role of genetic variation in nicotinemetabolizing enzymes. J.Neurogenet., 23(1563-526o (Electronic)):252-261, 2009. 
[59] Giovanni G. Di, E. Esposito, and Matteo Di. Role of serotonin in central dopamine dysfunction. CNS.Neurosci.Ther., 16(1755-5949 (Electronic)):179-194, June 2010.

[6o] F. Berrendero, P. Robledo, J.M. Trigo, E. Martin-Garcia, and R. Maldonado. Neurobiological mechanisms involved in nicotine dependence and reward: participation of the endogenous opioid system. Neurosci.Biobehav.Rev., 35 (1873-7528 (Electronic)):220-231, November 2010.

[61] C.G. Wong, T. Bottiglieri, and O.C. Snead. GABA, gamma-hydroxybutyric acid, and neurological disease. Ann.Neurol., 54 Suppl 6(0364-5134 (Print)):S3-12, 2003.

[62] A. Barbon and S. Barlati. Glutamate receptor RNA editing in health and disease. Biochemistry (Mosc.), 76(16083040 (Electronic)):882-889, August 2011.

[63] R. Ray, R.A. Schnoll, and C. Lerman. Pharmacogenetics and smoking cessation with nicotine replacement therapy. Cns.Drugs, 21(1172-7047 (Print)):525-533, 2007.

[64] J.T. Hays and J.O. Ebbert. Bupropion for the treatment of tobacco dependence: guidelines for balancing risks and benefits. Cns.Drugs, 17(1172-7047 (Print)):71-83, 2003.

[65] K. Cahill, L.F. Stead, and T. Lancaster. Nicotine receptor partial agonists for smoking cessation. Cochrane.Database.Syst.Rev., 4(1469-493X (Electronic)):CDoo6103, 2012.

[66] M. Iordanidou, A. Tavridou, I. Petridis, S. Kyroglou, L. Kaklamanis, D. Christakadis, and V.G. Manolopoulos. Association of polymorphisms of the serotonergic system with smoking initiation in caucasians. Drug Alcohol Depend., 108:70-76, 2010. ISSN 1879-0046.

[67] D. Li, S.J. London, J. Liu, W. Lee, X. Jiang, D. Van Den Berg, A.W. Bergen, D. Nishita, N. Waleh, G.E. Swan, P. Gallaher, C.P. Chou, J.C. Shih, J.B. Unger, W.J. Gauderman, F. Gilliland, C.A. Johnson, and D.V. Conti. Association of the calcyon neuron-specific vesicular protein gene (CALY) with adolescent smoking initiation in china and california. Am.J.Epidemiol., 173(1476-6256 (Electronic)):1039-1048, May 2011.

[68] The Tobacco and Genetics consortium. Genome-wide meta-analyses identify multiple loci associated with smoking behavior. Nat.Genet., 42(1546-1718 (Electronic)):441-447, May 2010.

[69] C.C. Fernandes, A. Pinto-Duarte, J.A. Ribeiro, and A.M. Sebastiao. Postsynaptic action of brain-derived neurotrophic factor attenuates alpha7 nicotinic acetylcholine receptor-mediated responses in hippocampal interneurons. J.Neurosci., 28(1529-2401 (Electronic)):5611-5618, May 2008.

[70] C. Lerman, P.G. Shields, E.P. Wileyto, J. Audrain, A. Pinto, L. Hawk, S. Krishnan, R. Niaura, and L. Epstein. Pharmacogenetic investigation of smoking cessation treatment. Pharmacogenetics, $12(0960-314 \mathrm{X}$ (Print)):627-634, November 2002.

[71] C. Lerman, E.P. Wileyto, F. Patterson, M. Rukstalis, J. Audrain-McGovern, S. Restine, P.G. Shields, V. Kaufmann, D. Redden, N. Benowitz, and W.H. Berrettini. The functional mu opioid receptor (OPRM1) Asn4oAsp variant predicts short-term response to nicotine replacement therapy in a clinical trial. Pharmacogenomics.J., 4(1470-269X (Print)):184-192, 2004.

[72] M.R. Munafo, I.J. Matheson, and J. Flint. Association of the DRD2 gene Taq1A polymorphism and alcoholism: a meta-analysis of case-control studies and evidence of publication bias. Mol.Psychiatry, 12(1359-4184 (Print)): 454-461, May 2007.

[73] S.P. David, D.R. Strong, M.R. Munafo, R.A. Brown, E.E. Lloyd-Richardson, P.E. Wileyto, E.A. Evins, P.G. Shields, C. Lerman, and R. Niaura. Bupropion efficacy for smoking cessation is influenced by the DRD2 Taq1A polymorphism: analysis of pooled data from two clinical trials. Nicotine.Tob.Res., 9(1462-2203 (Print)):1251-1257, December 2007 .

[74] S.P. David, R.A. Brown, G.D. Papandonatos, C.W. Kahler, E.E. Lloyd-Richardson, M.R. Munafo, P.G. Shields, C. Lerman, D. Strong, J. McCaffery, and R. Niaura. Pharmacogenetic clinical trial of sustained-release bupropion for smoking cessation. Nicotine.Tob.Res., 9(1462-2203 (Print)):821-833, August 2007.

[75] M.A. Styn, T. Nukui, M. Romkes, K. Perkins, S.R. Land, and J.L. Weissfeld. The impact of genetic variation in DRD2 and SLC6A3 on smoking cessation in a cohort of participants 1 year after enrollment in a lung cancer screening study. Am.J.Med.Genet.B Neuropsychiatr.Genet., 150B(1552-485X (Electronic)):254-261, March 2009. 
[76] C. Lerman, C. Jepson, E.P. Wileyto, L.H. Epstein, M. Rukstalis, F. Patterson, V. Kaufmann, S. Restine, L. Hawk, R. Niaura, and W. Berrettini. Role of functional genetic variation in the dopamine d2 receptor (DRD2) in response to bupropion and nicotine replacement therapy for tobacco dependence: results of two randomized clinical trials. Neuropsychopharmacology, 31(0893-133X (Print)):231-242, January 2006.

[77] S.P. David, M.R. Munafo, M.F. Murphy, M. Proctor, R.T. Walton, and E.C. Johnstone. Genetic variation in the dopamine $\mathrm{d}_{4}$ receptor $\left(\mathrm{DRD}_{4}\right)$ gene and smoking cessation: follow-up of a randomised clinical trial of transdermal nicotine patch. Pharmacogenomics.J., 8(1473-1150 (Electronic)):122-128, April 2008.

[78] S. Colilla, C. Lerman, P.G. Shields, C. Jepson, M. Rukstalis, A. DeMichele, G. Bunin, B.L. Strom, and T.R. Rebbeck. Association of catechol-o-methyltransferase with smoking cessation in two independent studies of women. Pharmacogenet. Genomics, 15:393-398, June 2005.

[79] M.R. Munafo, E.C. Johnstone, D. Walther, G.R. Uhl, M.F. Murphy, and P. Aveyard. CHRNA3 rs1051730 genotype and short-term smoking cessation. Nicotine.Tob.Res., 13(1469-994X (Electronic)):982-988, October 2011.

[8o] D.V. Conti, W. Lee, D. Li, J. Liu, D. Van Den Berg, P.D. Thomas, A.W. Bergen, G.E. Swan, R.F. Tyndale, N.L. Benowitz, and C. Lerman. Nicotinic acetylcholine receptor beta2 subunit gene implicated in a systems-based candidate gene study of smoking cessation. Hum.Mol.Genet., 17(1460-2083 (Electronic)):2834-2848, September 2008.

[81] K.A. Perkins, C. Lerman, M. Mercincavage, C.A. Fonte, and J.L. Briski. Nicotinic acetylcholine receptor beta2 subunit (CHRNB2) gene and short-term ability to quit smoking in response to nicotine patch. Cancer Epidemiology Biomarkers and Prevention, 18(1538-7755 (Electronic)):2608-2612, October 2009.

[82] A.M. Lee, C. Jepson, E. Hoffmann, L. Epstein, L.W. Hawk, C. Lerman, and R.F. Tyndale. CYP2B6 genotype alters abstinence rates in a bupropion smoking cessation trial. Biol.Psychiatry, 62(ooo6-3223 (Print)):635-641, September 2007.

[83] M. Quaak, C.P. van Schayck, D.S. Postma, E.J. Wagena, and F.J. van Schooten. Genetic variants in the serotonin transporter influence the efficacy of bupropion and nortriptyline in smoking cessation. Addiction, 107(1360-0443 (Electronic)), June 2011.

[84] S.S. Yoon, Y.K. Kwon, M.R. Kim, I. Shim, K.J. Kim, M.H. Lee, Y.S. Lee, G.T. Golden, and C.H. Yang. Acupuncturemediated inhibition of ethanol-induced dopamine release in the rat nucleus accumbens through the GABAB receptor. Neurosci.Lett., 369(0304-3940 (Print)):234-238, October 2004.

[85] H.J. Park, S.T. Kim, D.H. Yoon, S.H. Jin, S.J. Lee, H.J. Lee, and S. Lim. The association between the DRD2 TaqI a polymorphism and smoking cessation in response to acupuncture in koreans. J.Altern.Complement Med., 11 (1075-5535 (Print)):401-405, June 2005.

[86] J.P. Dahl, C. Jepson, R. Levenson, E.P. Wileyto, F. Patterson, W.H. Berrettini, and C. Lerman. Interaction between variation in the $\mathrm{d} 2$ dopamine receptor (DRD2) and the neuronal calcium sensor-1 (FREQ) genes in predicting response to nicotine replacement therapy for tobacco dependence. Pharmacogenomics.J., 6(1470-269X (Print)):194199, May 2006.

[87] C. Lerman, P.G. Shields, E.P. Wileyto, J. Audrain, L.H. Hawk, A. Pinto, S. Kucharski, S. Krishnan, R. Niaura, and L.H. Epstein. Effects of dopamine transporter and receptor polymorphisms on smoking cessation in a bupropion clinical trial. Health Psychol., 22(o278-6133 (Print)):541-548, September 2003.

[88] Advancing knowledge on regulating tobacco products, oslo, norway. Tob.Control, 9(2):224-226, June 2000.

[89] M. Rabinoff, N. Caskey, A. Rissling, and C. Park. Pharmacological and chemical effects of cigarette additives. Am.J.Public Health, 97(1541-0048 (Electronic)):1981-1991, November 2007.

[9o] S. Kassin. Psychology (Fourth Edition. Prentice-Hall, 2003.

[91] R.R. McCrae and P.T. Costa. Validation of the five-factor model of personality across instruments and observers. J.Pers.Soc.Psychol., 52(0022-3514 (Print)):81-90, January 1987.

[92] P.T. Costa and R.R. McCrae. Four ways five factors are basic. Personality and Individual Differences, 13(6):653-665, 1992.

[93] S. de Viron, J. Van der Heyden, E. Ambrosino, M. Arbyn, A. Brand, and H. Van Oyen. Impact of genetic notification on smoking cessation: Systematic review and pooled-analysis. Plos One, 7(7):e40230, July 2012. 
[94] WHO. Who report on the global tobacco epidemic. implementing smoke-free environments. Technical report, WHO, 2009

[95] N.S. Godtfredsen, M. Osler, J. Vestbo, I. Andersen, and E. Prescott. Smoking reduction, smoking cessation, and incidence of fatal and non-fatal myocardial infarction in denmark 1976-1998: a pooled cohort study. J.Epidemiol.Community Health, 57:412, 2003.

[96] W.B. White. Smoking-related morbidity and mortality in the cardiovascular setting. Prev.Cardiol., 10(1520-037X (Print)):1-4, 2007.

[97] B.W. Willemse, D.S. Postma, W. Timens, and N.H. ten Hacken. The impact of smoking cessation on respiratory symptoms, lung function, airway hyperresponsiveness and inflammation. Eur.Respir.J., 23(0903-1936 (Print)):464476, March 2004

[98] U. Broms, K. Silventoinen, P.A. Madden, A.C. Heath, and J. Kaprio. Genetic architecture of smoking behavior: a study of finnish adult twins. Twin.Res.Hum.Genet., 9(1832-4274 (Print)):64-72, February 2006.

[99] K.S. Kendler, L.M. Thornton, and N.L. Pedersen. Tobacco consumption in swedish twins reared apart and reared together. Arch.Gen.Psychiatry, 57(0003-990X (Print)):886-892, September 2000.

[10o] Y. Du and Y.J. Wan. The interaction of reward genes with environmental factors in contribution to alcoholism in mexican americans. Alcohol Clin.Exp.Res., 33(1530-0277 (Electronic)):2103-2112, December 2009.

[101] R. Plomin, J.C. Defries, G.E. McClearn, and P. McGuffin. Behavioral genetics. Worth Publishers, 2008.

[102] G.T. Fong, K.M. Cummings, R. Borland, G. Hastings, A. Hyland, G.A. Giovino, D. Hammond, and M.E. Thompson. The conceptual framework of the international tobacco control (ITC) policy evaluation project. Tob.Control, 15 Suppl 3(1468-3318 (Electronic)):iii3-11, June 2006.

[103] C. Manfredi, Y.I. Cho, K.S. Crittenden, and T.A. Dolecek. A path model of smoking cessation in women smokers of low socio-economic status. Health Education Research, 22(o268-1153 (Print)):747-756, October 2007.

[104] M. Talikka, N. Sierro, N.V. Ivanov, N. Chaudgary, M.J. Peck, J. Hoeng, C.R. Coggins, and M.C. Peitsch. Genomic impact of cigarette smoke, with application to three smoking-related diseases. Crit.Rev.Toxicol., 42:877-889, 2012.

[105] CDC. Cigarette smoking among adults — united states, 2007. MMWMR, 57(45):1221-1226, November 2008.

[106] J.R. Hughes, J. Keely, and S. Naud. Shape of the relapse curve and long-term abstinence among untreated smokers. Addiction, 99(0965-2140 (Print)):29-38, January 2004.

[107] S. Galea, M. Riddle, and G.A. Kaplan. Causal thinking and complex system approaches in epidemiology. Addiction, 39:97-106, 2010

[108] K.J. Rothman and S. Greenland. Causation and causal inference in epidemiology. Am.J.Public Health, 95 Suppl 1 (oogo-0036 (Print)):S144-S150, 2005.

[109] M.F. Hovell and S.C. Hughes. The behavioral ecology of secondhand smoke exposure: A pathway to complete tobacco control. Nicotine.Tob.Res., 11(1469-994X (Electronic)):1254-1264, November 2009.

[110] K. Honjo, A. Tsutsumi, I. Kawachi, and N. Kawakami. What accounts for the relationship between social class and smoking cessation? results of a path analysis. Soc.Sci.Med., 62(0277-9536 (Print)):317-328, January 2006.

[111] C.E. Lerman, R.A. Schnoll, and M.R. Munafo. Genetics and smoking cessation improving outcomes in smokers at risk. Am.J.Prev.Med., 33(0749-3797 (Print)):S398-S405, December 2007.

[112] G.E. Swan, K.S. Hudmon, L.M. Jack, K. Hemberger, D. Carmelli, T.V. Khroyan, H.Z. Ring, H. Hops, J.A. Andrews, E. Tildesley, D. McBride, N. Benowitz, C. Webster, K.C. Wilhelmsen, H.S. Feiler, B. Koenig, L. Caron, J. Illes, and L.S. Cheng. Environmental and genetic determinants of tobacco use: methodology for a multidisciplinary, longitudinal family-based investigation. Cancer Epidemiology Biomarkers and Prevention, 12(1055-9965 (Print)):9941005, October 2003

[113] P. Caponnetto and R. Polosa. Common predictors of smoking cessation in clinical practice. Respir. Med., 102: 1182-1192, 2008.

[114] J. Bryant, B. Bonevski, C. Paul, P. Mcelduff, and J. Attia. A systematic review and meta-analysis of the effectiveness of behavioural smoking cessation interventions in selected disadvantaged groups. Addiction, 106(1360-0443 (Electronic)):1568-1585, September 2011. 
[115] D. Wang, M. Connock, P. Barton, A. Fry-Smith, P. Aveyard, and D. Moore. 'Cut down to quit' with nicotine replacement therapies in smoking cessation: a systematic review of effectiveness and economic analysis. Health Technol.Assess., 12(1366-5278 (Print)):iii-xi, 1, February 2008.

[116] T. Coleman, S. Agboola, J. Leonardi-Bee, M. Taylor, A. McEwen, and A. McNeill. Relapse prevention in UK stop smoking services: current practice, systematic reviews of effectiveness and cost-effectiveness analysis. Health Technol.Assess., 14(1366-5278 (Print)):1-iv, October 2010.

[117] A. Cepeda-Benito, J.T. Reynoso, and S. Erath. Meta-analysis of the efficacy of nicotine replacement therapy for smoking cessation: differences between men and women. J.Consult Clin.Psychol., 72(0022-0o6X (Print)):712-722, August 2004.

[118] N.F. Woolacott, L. Jones, C.A. Forbes, L.C. Mather, A.J. Sowden, F.J. Song, J.P. Raftery, P.N. Aveyard, C.J. Hyde, and P.M. Barton. The clinical effectiveness and cost-effectiveness of bupropion and nicotine replacement therapy for smoking cessation: a systematic review and economic evaluation. Health Technol.Assess., 6(1366-5278 (Print)): $1-245,2002$.

[119] D. Moore, P. Aveyard, M. Connock, D. Wang, A. Fry-Smith, and P. Barton. Effectiveness and safety of nicotine replacement therapy assisted reduction to stop smoking: systematic review and meta-analysis. British Medical Journal, 338(1756-1833 (Electronic)):b1024, 2009.

[120] L.F. Stead, R. Perera, C. Bullen, D. Mant, and T. Lancaster. Nicotine replacement therapy for smoking cessation. Cochrane.Database.Syst.Rev., 23(1469-493X (Electronic)):CDooo146, 2008.

[121] J.R. Hughes, S. Shiffman, P. Callas, and J. Zhang. A meta-analysis of the efficacy of over-the-counter nicotine replacement. Tob.Control, 12:21-27, 2003.

[122] K.A. Perkins and J. Scott. Sex differences in long-term smoking cessation rates due to nicotine patch. Nicotine.Tob.Res., 10(1462-2203 (Print)):1245-1250, July 2008

[123] M.J. Eisenberg, K.B. Filion, D. Yavin, P. Belisle, S. Mottillo, L. Joseph, A. Gervais, J. O'Loughlin, G. Paradis, S. Rinfret, and L. Pilote. Pharmacotherapies for smoking cessation: a meta-analysis of randomized controlled trials. CMAJ., 179(1488-2329 (Electronic)):135-144, July 2008.

[124] S. Shiffman and S.G. Ferguson. Nicotine patch therapy prior to quitting smoking: a meta-analysis. Addiction, 103 (og65-2140 (Print)):557-563, April 2008.

[125] N. Lindson and P. Aveyard. An updated meta-analysis of nicotine preloading for smoking cessation: investigating mediators of the effect. Psychopharmacology (Berl), 214(1432-2072 (Electronic)):579-592, April 2011.

[126] J.R. Hughes, L.F. Stead, and T. Lancaster. Antidepressants for smoking cessation. Cochrane.Database.Syst.Rev., (1469-493X (Electronic)):CDoooo31, 2007.

[127] J.F. Etter. Cytisine for smoking cessation: a literature review and a meta-analysis. Arch.Intern.Med., 166(0003-9926 (Print)):1553-1559, August 2006.

[128] S. David, T. Lancaster, L.F. Stead, and A.E. Evins. Opioid antagonists for smoking cessation. Cochrane.Database.Syst.Rev., (1469-493X (Electronic)):CDoo3086, 2006.

[129] S.G. Gourlay, L.F. Stead, and N.L. Benowitz. Clonidine for smoking cessation. Cochrane.Database.Syst.Rev., (1469493X (Electronic)):CDoooo58, 2004.

[130] T. Asfar, J.O. Ebbert, R.C. Klesges, and G.E. Relyea. Do smoking reduction interventions promote cessation in smokers not ready to quit? Addict.Behav, 36:764-768, 2011.

[131] S. Papadakis, P. Mcdonald, K.A. Mullen, R. Reid, K. Skulsky, and A. Pipe. Strategies to increase the delivery of smoking cessation treatments in primary care settings: a systematic review and meta-analysis. Prev.Med., 51 (1096-0260 (Electronic)):199-213, September 2010.

[132] S.D. Shah, L.A. Wilken, S.R. Winkler, and S.J. Lin. Systematic review and meta-analysis of combination therapy for smoking cessation. J.Am.Pharm.Assoc.(2003.), 48(1544-3450 (Electronic)):659-665, September 2008.

[133] T. Lancaster and L.F. Stead. Individual behavioural counselling for smoking cessation. Cochrane.Database.Syst.Rev., (1469-493X (Electronic)):CDoo1292, 2005. 
[134] S. Mottillo, K.B. Filion, P. Belisle, L. Joseph, A. Gervais, J. O'Loughlin, G. Paradis, R. Pihl, L. Pilote, S. Rinfret, M. Tremblay, and M.J. Eisenberg. Behavioural interventions for smoking cessation: a meta-analysis of randomized controlled trials. Eur.Heart J., 30(1522-9645 (Electronic)):718-730, March 2009.

[135] L.F. Stead and T. Lancaster. Group behaviour therapy programmes for smoking cessation. Cochrane.Database.Syst.Rev., (1469-493X (Electronic)):CDoo1007, 2005.

[136] L.F. Stead, R. Perera, and T. Lancaster. Telephone counselling for smoking cessation. Cochrane.Database.Syst.Rev., 3(1469-493X (Electronic)):CDoo2850, 2006.

[137] L.F. Stead, R. Perera, and T. Lancaster. A systematic review of interventions for smokers who contact quitlines. Tob.Control, 16 Suppl 1(1468-3318 (Electronic)):i3-i8, December 2007.

[138] J.E. Hettema and P.S. Hendricks. Motivational interviewing for smoking cessation: a meta-analytic review. J.Consult Clin.Psychol., 78(1939-2117 (Electronic)):868-884, December 2010.

[139] C.J. Heckman, B.L. Egleston, and M.T. Hofmann. Efficacy of motivational interviewing for smoking cessation: a systematic review and meta-analysis. Tob.Control, 19(1468-3318 (Electronic)):410-416, October 2010.

[140] M.E. Bodner and E. Dean. Advice as a smoking cessation strategy: a systematic review and implications for physical therapists. Physiother.Theory.Pract., 25(1532-5040 (Electronic)):369-407, July 2009.

[141] P. Aveyard, R. Begh, A. Parsons, and R. West. Brief opportunistic smoking cessation interventions: a systematic review and meta-analysis to compare advice to quit and offer of assistance. Addiction, 107(1360-0443 (Electronic)): 1066-1073, June 2012.

[142] W.A. Mojica, M.J. Suttorp, S.E. Sherman, S.C. Morton, E.A. Roth, M.A. Maglione, S.L. Rhodes, and P.G. Shekelle. Smoking-cessation interventions by type of provider: a meta-analysis. Am.J.Prev.Med., 26(o749-3797 (Print)):391401, June 2004.

[143] K.V. Carson, M.E. Verbiest, M.R. Crone, M.P. Brinn, A.J. Esterman, W.J. Assendelft, and B.J. Smith. Training health professionals in smoking cessation. Cochrane.Database.Syst.Rev., 5(1469-493X (Electronic)):CDooo214, 2012.

[144] L.F. Stead, G. Bergson, and T. Lancaster. Physician advice for smoking cessation. Cochrane.Database.Syst.Rev., (1469-493X (Electronic)):CDooo165, 2008.

[145] V.H. Rice and L.F. Stead. Nursing interventions for smoking cessation. Cochrane.Database.Syst.Rev., (1469-493X (Electronic)):CDoo1188, 2008.

[146] V.H. Rice and L. Stead. Nursing intervention and smoking cessation: meta-analysis update. Heart Lung, 35 (o147-9563 (Print)):147-163, May 2006.

[147] R. Bize, B. Burnand, Y. Mueller, and J. Cornuz. Effectiveness of biomedical risk assessment as an aid for smoking cessation: a systematic review. Tob.Control, 16(1468-3318 (Electronic)):151-156, June 2007.

[148] R. Bize, B. Burnand, Y. Mueller, Walther M. Rege, and J. Cornuz. Biomedical risk assessment as an aid for smoking cessation. Cochrane.Database.Syst.Rev., (1469-493X (Electronic)):CDoo4705, 2009.

[149] P. Hajek and L.F. Stead. Aversive smoking for smoking cessation. Cochrane.Database.Syst.Rev., (1469-493X (Electronic)):CDooo546, 2004.

[150] M. Tahiri, S. Mottillo, L. Joseph, L. Pilote, and M.J. Eisenberg. Alternative smoking cessation aids: a meta-analysis of randomized controlled trials. Am.J.Med., 125:576-584, 2012.

[151] A.R. White, H. Rampes, and J.L. Campbell. Acupuncture and related interventions for smoking cessation. Cochrane database of systematic reviews (Online), (1):CDooooo9, 2011. ISSN 1469-493X.

[152] L. Shabab and A. McEwen. Online supportfor smoking cessation: a systematic review of the literature. Tob.Control, 13:1792-1804, 2009 .

[153] S.K. Myung, D.D. McDonnell, G. Kazinets, H.G. Seo, and J.M. Moskowitz. Effects of web- and computer-based smoking cessation programs: meta-analysis of randomized controlled trials. Arch.Intern.Med., 169(1538-3679 (Electronic)):929-937, May 2009.

[154] T. Lancaster and L.F. Stead. Self-help interventions for smoking cessation. Cochrane.Database.Syst.Rev., (1469-493X (Electronic)):CDoo1118, 2005. 
[155] C. Smerecnik, J.E. Grispen, and M. Quaak. Effectiveness of testing for genetic susceptibility to smoking-related diseases on smoking cessation outcomes: a systematic review and meta-analysis. Tob.Control, 21(3), May 2012.

[156] E.W. Park, J.K. Schultz, F. Tudiver, T. Campbell, and L. Becker. Enhancing partner support to improve smoking cessation. Cochrane.Database.Syst.Rev., (1469-493X (Electronic)):CDoo2928, 2012.

[157] E.W. Park, F. Tudiver, J.K. Schultz, and T. Campbell. Does enhancing partner support and interaction improve smoking cessation? a meta-analysis. Ann.Fam.Med., 2(1544-1709 (Print)):170-174, March 2004.

[158] K.D. Leeks, D.P. Hopkins, R.E. Soler, A. Aten, and S.K. Chattopadhyay. Worksite-based incentives and competitions to reduce tobacco use. a systematic review. Am.J.Prev.Med., 38(1873-26o7 (Electronic)):S263-S274, February 2010.

[159] G. Smedslund, K.J. Fisher, S.M. Boles, and E. Lichtenstein. The effectiveness of workplace smoking cessation programmes: a meta-analysis of recent studies. Tob.Control, 13(1468-3318 (Electronic)):197-204, June 2004.

[16o] S. Cengelli, J. O'Loughlin, B. Lauzon, and J. Cornuz. A systematic review of longitudinal population-based studies on the predictors of smoking cessation in adolescent and young adult smokers. Tob.Control, 21(1468-3318 (Electronic)):355-362, May 2012.

[161] P. Bader, H.E. Travis, and H.A. Skinner. Knowledge synthesis of smoking cessation among employed and unemployed young adults. Am.J.Public Health, 97(1541-0048 (Electronic)):1434-1443, August 2007.

[162] E. Vangeli, J. Stapleton, E.S. Smit, R. Borland, and R. West. Predictors of attempts to stop smoking and their success in adult general population samples: a systematic review. Addiction, 106(1360-0443 (Electronic)):21102121, December 2011.

[163] K. Cahill, T. Lancaster, and N. Green. Stage-based interventions for smoking cessation. Cochrane.Database.Syst.Rev., (1469-493X (Electronic)):CDoo4492, 2010.

[164] R.P. Riemsma, J. Pattenden, C. Bridle, A.J. Sowden, L. Mather, I.S. Watt, and A. Walker. Systematic review of the effectiveness of stage based interventions to promote smoking cessation. British Medical Journal, 326(1756-1833 (Electronic)):1175-1177, May 2003.

[165] C.J. Gwaltney, J. Metrik, C.W. Kahler, and S. Shiffman. Self-efficacy and smoking cessation: a meta-analysis. Psychol.Addict.Behav., 23(0893-164X (Print)):56-66, March 2009.

[166] I. Torchalla, C.T. Okoli, J.L. Bottorff, A. Qu, N. Poole, and L. Greaves. Smoking cessation programs targeted to women: a systematic review. Women Health, 52(1541-0331 (Electronic)):32-54, February 2012.

[167] S.P. David, M.R. Munafo, M.F. Murphy, R.T. Walton, and E.C. Johnstone. The serotonin transporter 5-HTTLPR polymorphism and treatment response to nicotine patch: follow-up of a randomized controlled trial. Nicotine.Tob.Res., 9(1462-2203 (Print)):225-231, February 2007.

[168] S.P. David, E.C. Johnstone, M.F. Murphy, P. Aveyard, B. Guo, C. Lerman, and M.R. Munafo. Genetic variation in the serotonin pathway and smoking cessation with nicotine replacement therapy: new data from the patch in practice trial and pooled analyses. Drug Alcohol Depend., 98(0376-8716 (Print)):77-85, November 2008.

[169] M.R. Munafo, E.C. Johnstone, E.P. Wileyto, P.G. Shields, K.M. Elliot, and C. Lerman. Lack of association of 5-HTTLPR genotype with smoking cessation in a nicotine replacement therapy randomized trial. Cancer Epidemiology Biomarkers and Prevention, 15(1055-9965 (Print)):398-400, February 2006.

[170] M.R. Munafo, E.C. Johnstone, M.F. Murphy, and P. Aveyard. Lack of association of DRD2 rs1800497 (Taq1A) polymorphism with smoking cessation in a nicotine replacement therapy randomized trial. Nicotine.Tob.Res., 11 (1469-994X (Electronic)):404-407, April 2009.

[171] G.E. Swan, A.M. Valdes, H.Z. Ring, T.V. Khroyan, L.M. Jack, C.C. Ton, S.J. Curry, and T. McAfee. Dopamine receptor DRD2 genotype and smoking cessation outcome following treatment with bupropion SR. Pharmacogenomics.J., 5(1470-269X (Print)):21-29, 2005.

[172] C.S. Wilcox, E.P. Noble, and N. Oskooilar. ANKK1/DRD2 locus variants are associated with rimonabant efficacy in aiding smoking cessation: pilot data. J.Investig.Med., 59(1708-8267 (Electronic)):1280-1283, December 2011.

[173] A.M. Leventhal, S.P. David, M. Brightman, D. Strong, J.E. McGeary, R.A. Brown, E.E. Lloyd-Richardson, M. Munafo, G.R. Uhl, and R. Niaura. Dopamine $\mathrm{d}_{4}$ receptor gene variation moderates the efficacy of bupropion for smoking cessation. Pharmacogenomics.J., 12(1473-1150 (Electronic)):86-92, February 2012. 
[174] M.R. Munafo, E.C. Johnstone, B. Guo, M.F. Murphy, and P. Aveyard. Association of COMT Val1o8/158Met genotype with smoking cessation. Pharmacogenetics and genomics, 18(1744-6872 (Print)):121-128, February 2008.

[175] E.C. Johnstone, K.M. Elliot, S.P. David, M.F. Murphy, R.T. Walton, and M.R. Munafo. Association of COMT Val108/158Met genotype with smoking cessation in a nicotine replacement therapy randomized trial. Cancer Epidemiology Biomarkers and Prevention, 16(1055-9965 (Print)):1065-1069, June 2007.

[176] W.H. Berrettini, E.P. Wileyto, L. Epstein, S. Restine, L. Hawk, P. Shields, R. Niaura, and C. Lerman. Catecholo-methyltransferase (COMT) gene variants predict response to bupropion therapy for tobacco dependence. Biol.Psychiatry, 61(0006-3223 (Print)):111-118, January 2007.

[177] T. Spruell, G. Colavita, T. Donegan, M. Egawhary, M. Hurley, P. Aveyard, E.C. Johnstone, M.F. Murphy, and M.R. Munafo. Association between nicotinic acetylcholine receptor single nucleotide polymorphisms and smoking cessation. Nicotine Tob.Res., 14:993-997, 2012.

[178] A.B. Gold, E.P. Wileyto, A. Lori, D. Conti, J.F. Cubells, and C. Lerman. Pharmacogenetic association of the galanin receptor (GALR1) SNP rs2717162 with smoking cessation. Neuropsychopharmacology, 37(1740-634X (Electronic)): 1683-1688, June 2012.

[179] R. Ray, C. Jepson, E.P. Wileyto, J.P. Dahl, F. Patterson, M. Rukstalis, A. Pinto, W. Berrettini, and C. Lerman. Genetic variation in mu-opioid-receptor-interacting proteins and smoking cessation in a nicotine replacement therapy trial. Nicotine.Tob.Res., 9(1462-2203 (Print)):1237-1241, November 2007.

[180] G.R. Uhl, Q.R. Liu, T. Drgon, C. Johnson, D. Walther, and J.E. Rose. Molecular genetics of nicotine dependence and abstinence: whole genome association using 520,000 SNPs. BMC.Genet., 8(1471-2156 (Electronic)):10, 2007.

[181] T. Drgon, C. Johnson, D. Walther, A.P. Albino, J.E. Rose, and G.R. Uhl. Genome-wide association for smoking cessation success: participants in a trial with adjunctive denicotinized cigarettes. Mol.Med., 15(1528-3658 (Electronic)):268-274, July 2009.

[182] G.R. Uhl, Q.R. Liu, T. Drgon, C. Johnson, D. Walther, J.E. Rose, S.P. David, R. Niaura, and C. Lerman. Molecular genetics of successful smoking cessation: convergent genome-wide association study results. Arch.Gen.Psychiatry, 65(1538-3636 (Electronic)):683-693, June 2008.

[183] G.R. Uhl, T. Drgon, C. Johnson, M.F. Ramoni, F.M. Behm, and J.E. Rose. Genome-wide association for smoking cessation success in a trial of precessation nicotine replacement. Mol.Med., 16(1528-3658 (Electronic)):513-526, November 2010.

[184] D.F. Heitjan, M. Guo, R. Ray, E.P. Wileyto, L.H. Epstein, and C. Lerman. Identification of pharmacogenetic markers in smoking cessation therapy. Am.J.Med.Genet.B Neuropsychiatr.Genet., $147 \mathrm{~B}(1552-485 \mathrm{X}$ (Electronic)):712719, September 2008 .

[185] R. Ray, N. Mitra, D. Baldwin, M. Guo, F. Patterson, D.F. Heitjan, C. Jepson, E.P. Wileyto, J. Wei, T. Payne, J.Z. Ma, M.D. Li, and C. Lerman. Convergent evidence that choline acetyltransferase gene variation is associated with prospective smoking cessation and nicotine dependence. Neuropsychopharmacology, 35(1740-634X (Electronic)): 1374-1382, May 2010.

[186] W. Lee, A.W. Bergen, G.E. Swan, D. Li, J. Liu, P. Thomas, R.F. Tyndale, N.L. Benowitz, C. Lerman, and D.V. Conti. Gender-stratified gene and gene-treatment interactions in smoking cessation. Pharmacogenomics, 12:521-532, 2012.

[187] J.R. Hughes, L.F. Stead, and T. Lancaster. Nortriptyline for smoking cessation. Nicotine Tob.Res., 7:491-499, 2005.

[188] S. Holmes, N. Zwar, C.A. Jimenez-Ruiz, P.J. Ryan, D. Browning, L. Bergmann, and J.A. Johnston. Bupropion as an aid to smoking cessation: a review of real-life effectiveness. Int.J.Clin.Pract., 58(1368-5031 (Print)):285-291, March 2004 .

[189] N. Zwar and R. Richmond. Bupropion sustained release. a therapeutic review of zyban. Aust.Fam.Physician, 31: 443-447, 2002.

[19o] S.L. Zierler-Brown and J.A. Kyle. Oral varenicline for smoking cessation. Ann.Pharmacother., 41(1542-6270 (Electronic)):95-99, January 2007.

[191] T. Lancaster and L.F. Stead. Mecamylamine (a nicotine antagonist) for smoking cessation. Cochrane.Database.Syst.Rev., (1469-493X (Electronic)):CDoo10o9, 2000.

[192] K. Cahill and M.H. Ussher. Cannabinoid type 1 receptor antagonists for smoking cessation. Cochrane.Database.Syst.Rev., (1469-493X (Electronic)):CDoo5353, 2011. 
[193] K. Cahill and R. Perera. Competitions and incentives for smoking cessation. Cochrane.Database.Syst.Rev., (1469493X (Electronic)):CDoo4307, 2011.

[194] H.E. Hutton, L.M. Wilson, B.J. Apelberg, E.A. Tang, O. Odelola, E.B. Bass, and G. Chander. A systematic review of randomized controlled trials: Web-based interventions for smoking cessation among adolescents, college students, and adults. Nicotine.Tob.Res., 13(1469-994X (Electronic)):227-238, April 2011.

[195] M. Civljak, A. Sheikh, L.F. Stead, and J. Car. Internet-based interventions for smoking cessation. Cochrane.Database.Syst.Rev., (1469-493X (Electronic)):CDoo7078, 2010.

[196] F. Tzelepis, C.L. Walsh, P. Mcelduff, and J. Knight. Proactive telephone counseling for smoking cessation: metaanalyses by recruitment channel and methodological quality. J.Nat.Cancer, 103:922-941, 2011.

[197] W. Pan. Proactive telephone counseling as an adjunct to minimal intervention for smoking cessation: a metaanalysis. Health Education Research, 21(0268-1153 (Print)):416-427, June 2006.

[198] T. Mantler, J.D. Irwin, and D. Morrow. Motivational interviewing and smoking behaviors: a critical appraisal and literature review of selected cessation initiatives. Psychol.Rep., 110(0033-2941 (Print)):445-460, April 2012.

[199] J.B. McClure. Are biomarkers a useful aid in smoking cessation? a review and analysis of the literature. Behav.Med., 27(0896-4289 (Print)):37-47, 2001.

[200] K. Cahill and R. Perera. Quit and win contests for smoking cessation. Cochrane.Database.Syst.Rev., (1469-493X (Electronic)):CDoo4986, 2008.

[201] J.P. Green, Lynn S. Jay, and G.H. Montgomery. A meta-analysis of gender, smoking cessation, and hypnosis:a brief communication. International Journal of Clinical and Experimental Hypnosis, 54(0020-7144 (Print)):224-233, April 2006.

[202] J.P. Green, S.J. Lynn, and G.H. Montgomery. Gender-related differences in hypnosis-based treatments for smoking: a follow-up meta-analysis. Am.J.Clin.Hypn., 50(0002-9157 (Print)):259-271, January 2008.

[203] M.H. Ussher, A. Taylor, and G. Faulkner. Exercise interventions for smoking cessation. Cochrane.Database.Syst.Rev., 1(1469-493X (Electronic)):CDoo2295, 2012.

[204] M.H. Ussher, A.H. Taylor, R. West, and A. McEwen. Does exercise aid smoking cessation? a systematic review. Addiction, 95(0965-2140 (Print)):199-208, February 2000.

[205] S.M. Zbikowski, B. Magnusson, J.R. Pockey, H.A. Tindle, and K.E. Weaver. A review of smoking cessation interventions for smokers aged 50 and older. Maturitas, 71(1873-4111 (Electronic)):131-141, February 2012.

[206] S. May and R. West. Do social support interventions (buddy systems) aid smoking cessation? Tob.Control, 9: 415-422, 2000.

[207] K. Cahill, M. Moher, and T. Lancaster. Workplace interventions for smoking cessation. Cochrane.Database.Syst.Rev., (1469-493X (Electronic)):CDoo3440, 2008.

[208] L.M. Wilson, Tang E. Avila, G. Chander, H.E. Hutton, O.A. Odelola, J.L. Elf, B.M. Heckman-Stoddard, E.B. Bass, E.A. Little, E.B. Haberl, and B.J. Apelberg. Impact of tobacco control interventions on smoking initiation, cessation, and prevalence: a systematic review. J.Environ.Public Health, 2012(1687-9813 (Electronic)):961724, 2012.

[209] M. Bala, L. Strzeszynski, and K. Cahill. Mass media interventions for smoking cessation in adults. Cochrane.Database.Syst.Rev., (1469-493X (Electronic)):CDoo4704, 2008.

[210] J. Niederdeppe, X. Kuang, B. Crock, and A. Skelton. Media campaigns to promote smoking cessation among socioeconomically disadvantaged populations: what do we know, what do we need to learn, and what should we do now? Soc.Sci.Med., 67(0277-9536 (Print)):1343-1355, November 2008.

[211] D.P. Hopkins, S. Razi, K.D. Leeks, Kalra G. Priya, S.K. Chattopadhyay, and R.E. Soler. Smokefree policies to reduce tobacco use. a systematic review. Am.J.Prev.Med., 38(1873-2607 (Electronic)):S275-S289, February 2010.

[212] J.A. Alegria-Torres, A. Baccarelli, and V. Bollati. Epigenetics and lifestyle. Epigenomics., 3(1750-192X (Electronic)): 267-277, June 2011.

[213] M. Parascandola. Causes, risks, and probabilities: probabilistic concepts of causation in chronic disease epidemiology. Prev.Med., 53(1096-0260 (Electronic)):232-234, October 2011. 
[214] M.L. Pianezza, E.M. Sellers, and R.F. Tyndale. Nicotine metabolism defect reduces smoking. Nature, 393(00280836 (Print)):750, June 1998.

[215] R. Mermelstein. Ethnicity, gender and risk factors for smoking initiation: an overview. Nicotine.Tob.Res., 1 Suppl 2(1462-2203 (Print)):S39-43, discussion, 1999.

[216] J.D. Flom, J.S. Ferris, Y. Liao, P. Tehranifar, C.B. Richards, Y.H. Cho, K. Gonzalez, R.M. Santella, and M.B. Terry. Prenatal smoke exposure and genomic DNA methylation in a multiethnic birth cohort. Cancer Epidemiology Biomarkers and Prevention, 20(1538-7755 (Electronic)):2518-2523, December 2011.

[217] M.R. Munafo, T.G. Clark, L.R. Moore, E. Payne, R. Walton, and J. Flint. Genetic polymorphisms and personality in healthy adults: a systematic review and meta-analysis. Mol.Psychiatry, 8(1359-4184 (Print)):471-484, May 2003.

[218] J.M. Fletcher. Why have tobacco control policies stalled? using genetic moderation to examine policy impacts. PLoS One, 7:e50576, 2012.

[219] R. Pechey, D. Spiegelhalter, and T.M. Marteau. Impact of plain packaging of tobacco products pn smoking in adults and children: an elicitation of international experts' estimates. BMC Public Health, 13:18, 2013.

[220] MeSH, 2013. URL http://www.ncbi.nlm.nih.gov/mesh. accessed in April 2013.

[221] S. de Viron. Genetic predictors for smoking initiation and cessation, pages 98-114. DOI: 10.2217/EBO.12.233. Future Medicine, London, UK, 2013.

[222] A. Brand. Public health genomics - public health goes personalized? Eur.J.Public Health, 21(1464-360X (Electronic)):2-3, February 2011.

[223] K.L. Noblett and E.F. Coccaro. Molecular genetics of personality. Curr.Psychiatry Rep., 7(1523-3812 (Print)):73-80, March 2005.

[224] R.P. Ebstein. The molecular genetic architecture of human personality: beyond self-report questionnaires. Mol.Psychiatry, 11(1359-4184 (Print)):427-445, May 2006.

[225] A.J. Sasco, M.B. Secretan, and K. Straif. Tobacco smoking and cancer: a brief review of recent epidemiological evidence. Lung Cancer, 45 Suppl 2(0169-5002 (Print)):S3-S9, August 2004.

[226] J. de Leon and F.J. Diaz. A meta-analysis of worldwide studies demonstrates an association between schizophrenia and tobacco smoking behaviors. Schizophr.Res., 76(2-3):135-157, 2005.

[227] M.R. Munafo and R. Araya. Cigarette smoking and depression: a question of causation. Br.J.Psychiatry, 196(6): 425-426, 2010.

[228] A.S. Ade, Z.C. Wright, and D.J. States. Gene2MeSH [internet]. ann arbor (MI): national center for integrative biomedical informatics, 2007 .

[229] L.J. Jensen, M. Kuhn, M. Stark, S. Chaffron, C. Creevey, J. Muller, T. Doerks, P. Julien, A. Roth, M. Simonovic, P. Bork, and Mering C. von. STRING 8-a global view on proteins and their functional interactions in 630 organisms. Nucleic Acids Res., 37(1362-4962 (Electronic)):D412-D416, January 2009.

[230] Y. Kaneko, Y. Yatagai, H. Yamada, H. Iijima, H. Masuko, T. Sakamoto, and N. Hizawa. The search for common pathways underlying asthma and copd. Int.J.Chron.Obstruct.Pulmon.Dis., 8:65-78, 2013.

[231] S.N. Grief. Nicotine dependence: health consequences, smoking cessation therapies, and pharmacotherapy. Prim.Care, 38(1):23-39, 2011.

[232] D. Morisano, I. Bacher, J. Audrain-McGovern, and T.P. George. Treatment of smokers with co-occurring disorders: emphasis on integration in mental health and addiction treatment settings. Can.J.Psychiatry, 54(6), 2009.

[233] S.M. Hall and J.J. Prochaska. Mechanisms underlying the comorbidity of tobacco use in mental health and addictive disorders. Annu.Rev.Clin.Psychol., 5, 2009.

[234] C. Gotti, M. Zoli, and F. Clementi. Brain nicotinic acetylcholine receptors: native subtypes and their relevance. Trends Pharmacol.Sci., 27(0165-6147 (Print)):482-491, September 2006.

[235] T. Hayashi and T.P. Su. Sigma-1 receptor ligands: potential in the treatment of neuropsychiatric disorders. CNS.Drugs, 18(5):269-284, 2004. 
[236] L. Zeng, P. Zhang, L. Shi, V. Yamamoto, W. Lu, and K. Wang. Functional impacts of nrxn1 knockdown on neurodevelopment in stem cell models. PloS One, 8(3):e59685, 2013.

[237] F.J. McClernon and S.H. Kollins. ADHD and smoking: from genes to brain to behavior. Ann.N.Y.Acad.Sci., 1141 (1749-6632 (Electronic)):131-147, October 2008.

[238] R.C. McEachin, N.L. Saccone, S.F. Saccone, Y.D. Kleyman-Smith, T. Kar, R.K. Kare, A.S. Ade, M.A. Sartor, J.D. Cavalcoli, and M.G. McInnis. Modeling complex genetic and environmental influences on comorbid bipolar disorder with tobacco use disorder. BMC.Med.Genet., 11(1471-2350 (Electronic)):14, 2010.

[239] K.E. Joynt, D.J. Whellan, and C.M. O'Connor. Depression and cardiovascular disease: mechanisms of interaction. Biol.Psychiatry, 54(3):248-261, 2003.

[240] A. Meyer-Lindenberg and D.R. Weinberger. Intermediate phenotypes and genetic mechanisms of psychiatric disorders. Nat.Rev.Neurosci, 7(10):818-827, 2006.

[241] K. Blum, A.L. Chen, M. Oscar-Berman, T.J. Chen, J. Lubar, N. White, J. Lubar, A. Bowirrat, E. Braverman, J. Schoolfield, R.L. Waite, B.W. Downs, M. Madigan, D.E. Comings, C. Davis, M.M. Kerner, J. Knopf, T. Palomo, J.J. Giordano, S.A. Morse, F. Fornari, D. Barh, J. Femino, and J.A. Bailey. Generational association studies of dopaminergic genes in reward deficiency syndrome (RDS) subjects: selecting appropriate phenotypes for reward dependence behaviors. Int.J.Environ.Res.Public Health, 8(1660-4601 (Electronic)):4425-4459, December 2011.

[242] B.T. Conner, G.S. Hellemann, T.L. Ritchie, and E.P. Noble. Genetic, personality, and environmental predictors of drug use in adolescents. J.Subst.Abuse Treat., 38(1873-6483 (Electronic)):178-190, March 2010.

[243] C. Carlsten, A. Halperin, J. Crouch, and W. Burke. Personalized medicine and tobacco-related health disparities: is there a role for genetics? Ann.Fam.Med., 9(1544-1717 (Electronic)):366-371, July 2011.

[244] R. Walton, E. Johnstone, M. Munafo, M. Neville, and S. Griffiths. Genetic clues to the molecular basis of tobacco addiction and progress towards personalized therapy. Trends Mol.Med., 7(2):70-76, 2001.

[245] A. Peters and K. Katsouyanni. Understanding the link between environmental exposures and health: does the exposome promise too much? J.Epidemiol.Community Health, 66(2), 2012.

[246] CDC. How tobacco smoke causes disease: The biology and behavioral basis for smoking-attributable disease. NBK53017 [bookaccession], CDC, 2010.

[247] R.T. Croyle and C. Lerman. Risk communication in genetic testing for cancer susceptibility. J.Natl.Cancer Inst.Monogr, (1052-6773 (Print)):59-66, 1999.

[248] A. Trepanier, M. Ahrens, W. McKinnon, J. Peters, J. Stopfer, S.C. Grumet, S. Manley, J.O. Culver, R. Acton, J. Larsen-Haidle, L.A. Correia, R. Bennett, B. Pettersen, T.D. Ferlita, J.W. Costalas, K. Hunt, S. Donlon, C. Skrzynia, C. Farrell, F. Callif-Daley, and C.W. Vockley. Genetic cancer risk assessment and counseling: recommendations of the national society of genetic counselors. J.Genet.Couns., 13(1059-7700 (Print)):83-114, April 2004.

[249] A.K. Alexandrie, F. Nyberg, M. Warholm, and A. Rannug. Influence of CYP1A1, GSTM1, GSTT1, and NQO1 genotypes and cumulative smoking dose on lung cancer risk in a swedish population. Cancer Epidemiology Biomarkers and Prevention, 13(1055-9965 (Print)):908-914, June 2004.

[250] M. Sorensen, H. Autrup, A. Tjonneland, K. Overvad, and O. Raaschou-Nielsen. Glutathione s-transferase tı nullgenotype is associated with an increased risk of lung cancer. Int.J.Cancer, 110(oo20-7136 (Print)):219-224, June 2004 .

[251] X. Shi, S. Zhou, Z. Wang, Z. Zhou, and Z. Wang. CYP1A1 and GSTM1 polymorphisms and lung cancer risk in chinese populations: a meta-analysis. Lung Cancer, 59(0169-5002 (Print)):155-163, February 2008.

[252] M. Spinola, F.S. Falvella, A. Galvan, C. Pignatiello, V.P. Leoni, U. Pastorino, R. Paroni, S. Chen, V. Skaug, A. Haugen, and T.A. Dragani. Ethnic differences in frequencies of gene polymorphisms in the MYCL1 region and modulation of lung cancer patients' survival. Lung Cancer, 55(0169-5002 (Print)):271-277, March 2007.

[253] P.M. Christensen, P.C. Gotzsche, and K. Brosen. The sparteine/debrisoquine (CYP2D6) oxidation polymorphism and the risk of lung cancer: a meta-analysis. Eur.J.Clin.Pharmacol., 51(5):389-393, 1997.

[254] K. Sorensen and H. Brand. Health literacy: the essential catalyst for the responsible and effective translation of genome-based information for the benefit of population health. Public Health Genomics, 14(1662-8063 (Electronic)): 195-200, 2011. doi: 10.1159/000324241. 
[255] I.M. Lipkus. Numeric, verbal, and visual formats of conveying health risks: suggested best practices and future recommendations. Med.Decis.Making, 27(0272-989X (Print)):696-713, September 2007.

[256] K. Witte. Putting the fear back into fear appeals: The extended parallel process mode. Commun. Monogr., 59: 329-349, 1992.

[257] S.B. Haga. Ethical issues of predictive genetic testing for diabetes. J.Diabetes Sci.Technol., 3(1932-2968 (Electronic)): 781-788, July 2009

[258] A. Liberati, D.G. Altman, J. Tetzlaff, C. Mulrow, P.C. Gotzsche, J.P.A. Ioannidis, M. Clarke, P.J. Devereaux, J. Kleijnen, and D. Moher. The PRISMA statement for reporting systematic reviews and meta-analyses of studies that evaluate healthcare interventions: explanation and elaboration. British Medical Journal, 339(jul21_1):b270o, December 2009.

[259] W.F. Velicer and J.O. Prochaska. A comparison of four self-report smoking cessation outcome measures. Addict.Behav., 29(0306-4603 (Print)):51-60, January 2004

[26o] J. Audrain, N.R. Boyd, J. Roth, D. Main, N.E. Caporaso, and C. Lerman. Genetic susceptibility testing in smokingcessation treatment: One-year outcomes of a randomized trial. Addictive Behaviors, 22(6):741-751, 1997. ISSN 0306-4603.

[261] C. Lerman, K. Gold, J. Audrain, T.H. Lin, N.R. Boyd, C.T. Orleans, B. Wilfond, G. Louben, and N. Caporaso. Incorporating biomarkers of exposure and genetic susceptibility into smoking cessation treatment: effects on smoking-related cognitions, emotions, and behavior change. Health Psychol., 16(o278-6133 (Print)):87-99, January 1997

[262] S.C. Sanderson, S.E. Humphries, C. Hubbart, E. Hughes, M.J. Jarvis, and J. Wardle. Psychological and behavioural impact of genetic testing smokers for lung cancer risk: a phase II exploratory trial. J.Health Psychol., 13(1359-1053 (Print)):481-494, May 2008.

[263] R.P. Harris, M. Helfand, S.H. Woolf, K.N. Lohr, C.D. Mulrow, S.M. Teutsch, and D. Atkins. Current methods of the US preventive services task force: a review of the process. Am.J.Prev.Med., 20(o749-3797 (Print)):21-35, April 2001

[264] R. Dersimonian and N. Laird. Meta-analysis in clinical trials. Control Clin.Trials, 7(0197-2456 (Print)):177-188, September 1986.

[265] J.P. Higgins, S.G. Thompson, J.J. Deeks, and D.G. Altman. Measuring inconsistency in meta-analyses. British Medical Journal, 327(1468-5833 (Electronic)):557-56o, September 2003.

[266] A. Tobias. sbe26: Assesing the influence of a single study in the meta-analysis estimate. Stata Techn Bull, 47:15-17, 1999.

[267] S.C. Sanderson and S. Michie. Genetic testing for heart disease susceptibility: potential impact on motivation to quit smoking. Clin.Genet., 71(0oo9-9163 (Print)):501-510, June 2007.

[268] S.C. Sanderson and J. Wardle. Will genetic testing for complex diseases increase motivation to quit smoking? anticipated reactions in a survey of smokers. Health Education and Behavior, 32(1090-1981 (Print)):640-653, October 2005 .

[269] J.L. Westmaas and P.B. Woicik. Dispositional motivations and genetic risk feedback. Addict.Behav., 30(0306-4603 (Print)):1524-1534, September 2005.

[270] A.J. Wright, P. Aveyard, B. Guo, M. Murphy, K. Brown, and T.M. Marteau. Is attributing smoking to genetic causes associated with a reduced probability of quit attempt success? a cohort study. Addiction, 102(0965-2140 (Print)):1657-1664, October 2007.

[271] M.J. Carpenter, C. Strange, Y. Jones, M.R. Dickson, C. Carter, M.A. Moseley, and G.E. Gilbert. Does genetic testing result in behavioral health change? changes in smoking behavior following testing for alpha-1 antitrypsin deficiency. Ann.Behav.Med., 33(o883-6612 (Print)):22-28, February 2007.

[272] I.M. Lipkus, C.M. McBride, K.I. Pollak, P. Lyna, and G. Bepler. Interpretation of genetic risk feedback among african american smokers with low socioeconomic status. Health Psychol., 23:177-188, 2004.

[273] S.C. Sanderson, S.C. O’Neill, D.B. White, G. Bepler, L. Bastian, I.M. Lipkus, and C.M. McBride. Responses to online GSTM1 genetic test results among smokers related to patients with lung cancer: a pilot study. Cancer Epidemiology Biomarkers and Prevention, 18(1538-7755 (Electronic)):1953-1961, July 2009. 
[274] G.R. Uhl, T. Drgon, C. Johnson, and J.E. Rose. Nicotine abstinence genotyping: assessing the impact on smoking cessation clinical trials. Pharmacogenomics.J., 9(1473-1150 (Electronic)):111-115, April 2009.

[275] J.O. Prochaska and C.C. Diclemente. Stages and processes of self-change of smoking: toward an integrative model of change. J.Consult Clin.Psychol., 51(0022-006X (Print)):390-395, June 1983.

[276] M.R. Munafo, T.G. Clark, and J. Flint. Assessing publication bias in genetic association studies: evidence from a recent meta-analysis. Psychiatry Res., 129(0165-1781 (Print)):39-44, November 2004.

[277] K.P. Sajadi and H.B. Goldman. Social networks lack useful content for incontinence. Urology, 78(1527-9995 (Electronic)):764-767, October 2011.

[278] B. Freeman. New media and tobacco control. Tob.Control, 21(1468-3318 (Electronic)):139-144, March 2012.

[279] Social media 'likes' healthcare: From marketing to social business. Technical report, PwC, 2012.

[280] B.P. Jenssen, J.D. Klein, L.F. Salazar, N.A. Daluga, and R.J. DiClemente. Exposure to tobacco on the internet: content analysis of adolescents' internet use. Pediatrics, 124(1098-4275 (Electronic)):e180-e186, August 2009.

[281] M. Hefler, B. Freeman, and S. Chapman. Tobacco control advocacy in the age of social media: using facebook, twitter and change. Tob.Control, (3):210-214, 2012.

[282] A.C. Reaves and D.W. Bianchi. The role of social networking sites in medical genetics research. Am.J.Med.Genet., 161:951-957, 2013.

[283] Alexa: The web information company, 2012. URL http://alexa.com. accessed in October 2012.

[284] Facebook statistics, 2012. URL https://www. facebook.com/photo.php?fbid=10151498158461729\&set=a. 10151498156121729.515066. 20531316728\&type=3\&src=https $\% 3 \mathrm{~A} \% 2 \mathrm{~F} \% 2 \mathrm{Ffbcdn}-$ sphotos $-\mathrm{h}-\mathrm{a}$. akamaind . net $\%$ 2Fhphotos - ak-prn1\%2F485678_10151498158461729_805685678_n.jpg\&size=960\%2C720. accessed in February 2013.

[285] Twitter advertising, 2013. URL https://twitter.com/TwitterAds. accessed in February 2013.

[286] Youtube statistics, 2013. URL http://www.youtube.com/t/press_statistics. accessed in February 2013.

[287] C.I. Amos, X. Wu, I.P. Gorlov, J. Gu, T. Eisen, Q. Dong, Q. Zhang, X. Gu, J. Vijayakrishnan, K. Sullivan, A. Matakidou, Y. Wang, G. Mills, K. Doheny, Y.Y. Tsai, W.V. Chen, S. Shete, M.R. Spitz, and R.S. Houlston. Genome-wide association scan of tag snps identifies a susceptibility locus for lung cancer at 15q25.1. Nat.Genet., 40:616-622, 2008.

[288] R. Govindan, L. Ding, M. Griffith, J. Subramanian, N.D. Dees, K.L. Kanchi, C.A. Maher, R. Fulton, L. Fulton, J. Wallis, K. Chen, J. Walker, S. McDonald, R. Bose, D. Ornitz, D. Xiong, M. You, D.J. Dooling, M. Watson, Mardis E.R., and Wilson R.K. Genomic landscape of non-small cell lung cancer in smokers and never-smokers. Cell, 150: $1121-1134,2012$.

[289] D.W. Belsky, T.E. Moffitt, T.B. Baker, A.K. Biddle, J.P. Evans, H. Harrington, R. Houts, M. Meier, K. Sugden, B. Williams, R. Poulton, and Caspi A. Polygenic risk and the developmental progression to heavy, persistent smoking and nicotine dependence: evidence from a 4-decade longitudinal study. JAMA Psychiatry, 70:534-542, 2013.

[290] Internet world stats, 2012. URL http://www. internetworldstats. com/stats.htm. accessed in October 2012.

[291] Socialbakers, 2012. URL http://www. socialbakers.com/. accessed in October 2012.

[292] V. Zazzu, B. Regierer, A. Kuhn, R. Sudbrak, and H. Lehrach. It future of medicine: from molecular analysis to clinical diagnosis and improved treatment. N.Biotechnol., 30(4):362-365, 2012.

[293] European alliance for personalized medicine, 2012. URL www. euapm. eu. accessed in February 2013.

[294] A.E. Simon, D. Juszczyk, N. Smyth, E. Power, S. Hiom, M.D. Peake, and J. Wardle. Knowledge of lung cancer symptoms and risk factors in the u.k.: development of a measure and results from a population-based survey. Thorax, 67:426-432, 2012.

[295] Tang D.W., Hello B., Mroziewicz M., Fellows L.K., Tyndale R.F., and A. Dagher. Genetic variation in cyp2a6 predicts neural reactivity to smoking cues as measured using fMRI. Neuroimage, 60:2136-2143, 2012. 
[296] L. Pray. Genes, smoking, and lung cancer. Nature Education, 1, 2008.

[297] D.H. Lea, K.A. Kaphingst, D. Bowen, I. Lipkus, and D.W. Hadley. Communicating genetic and genomic information: health literacy and numeracy considerations. Public Health Genomics, 14(1662-8063 (Electronic)):279-289, 2011.

[298] S.R. Cotten and S.S. Gupta. Characteristics of online and offline health information seekers and factors that discriminate between them. Soc.Sci.Med., 59(9):1795-1806, 2004.

[299] H. Korda and Z. Itani. Harnessing social media for health promotion and behavior change. Health Promot.Pract., 14(1):15-23, 2013.

[30o] S. Fox. Health topics: $80 \%$ of users look for health information online. Technical report, Pew Internet \& American Life Project, 2011.

[301] B.W. Hesse, R.P. Moser, and L.J. Rutten. Surveys of physicians and electronic health information. N.Engl.J.Med., $362(9): 859-860,2010$.

[302] N. Elkin. How america searches: Health and wellness. Technical report, iCrossing, 2008.

[303] T.L. Webb, J. Joseph, L. Yardley, and S. Michie. Using the internet to promote health behavior change: a systematic review and meta-analysis of the impact of theoretical basis, use of behavior change techniques, and mode of delivery on efficacy. J.Med.Internet Res., 12(1):e4, 2010.

[304] S. de Viron, L.S. Suggs, A. Brand, and H. Van Oyen. Communicating genetics and smoking through social media: Are we there yet? J. Med. Internet Res., 15:e198, 2013.

[305] J.D. Johnson, J.E. Andrews, and S. Allard. A model for understanding and affecting cancer genetics information seeking. Library and Information science research, 23(4):335-349, 2001.

[306] B. Niedzwiedzka. A proposed general model of information behaviour. Information research, 9(1):paper 164, 2003.

[307] R.E. Rice. Influences, usage, and outcomes of internet health information searching: multivariate results from the pew surveys. Int.J.Med.inform., 75(1):8-28, 2006.

[308] W.Y. Chou, Y.M. Hunt, E.B. Beckjord, R.P. Moser, and B.W. Hesse. Social media use in the united states: implications for health communication. J.Med.Internet.Res., 11(1438-8871 (Electronic)):e48, 2009.

[309] M.D. Kaplowitz, F. Lupi, M.P. Couper, and L. Thorp. The effect of invitiation design on web survey response rates. Social Science Computer Review, 30(3):339-349, 2012.

[310] N. Solovieff, C. Cotsapas, P.H. Lee, S.M. Purcell, and J.W. Smoller. Pleiotropy in complex traits: challenges and strategies. Nature Reviews Genetics, 14:483-495, 2013.

[311] N. Herd, R. Borland, and A. Hyland. Predictors of smoking relapse by duration of abstinence: findings from the internation tobacco control(ITC) four country survey. Addiction, 104:2088-2099, 2009. 